\title{
Crafting Matter-Reality
}

\author{
by \\ Patrick G. Bisson - Master Of Architecture
}

\author{
A thesis submitted to \\ The Faculty of Graduate Studies \\ in partial fulfillment of \\ the requirements for the degree of
}

\section{MASTER OF ARCHITECTURE}

M.ARCH. (Professional)

\author{
CARLETON UNIVERSITY \\ Ottawa, Ontario \\ September 19th, 2008 \\ (C) copyright \\ 2008, Patrick Bisson
}


Library and

Archives Canada

Published Heritage

Branch

395 Wellington Street

Ottawa ON K1A ON4

Canada
Bibliothèque et

Archives Canada

Direction du

Patrimoine de l'édition

978-0-494-44099-5

395 , rue Wellington

Ottawa ON K1A ON4

Canada
Yourfile Votre référence
ISBN:
Our file Notre retérence
ISBN:
NOTICE:

The author has granted a nonexclusive license allowing Library and Archives Canada to reproduce, publish, archive, preserve, conserve, communicate to the public by telecommunication or on the Internet, loan, distribute and sell theses worldwide, for commercial or noncommercial purposes, in microform, paper, electronic and/or any other formats.

The author retains copyright ownership and moral rights in this thesis. Neither the thesis nor substantial extracts from it may be printed or otherwise reproduced without the author's permission.
AVIS:

L'auteur a accordé une licence non exclusive permettant à la Bibliothèque et Archives Canada de reproduire, publier, archiver, sauvegarder, conserver, transmettre au public par télécommunication ou par l'Internet, prêter, distribuer et vendre des thèses partout dans le monde, à des fins commerciales ou autres, sur support microforme, papier, électronique et/ou autres formats.

L'auteur conserve la propriété du droit d'auteur et des droits moraux qui protège cette thèse. $\mathrm{Ni}$ la thèse ni des extraits substantiels de celle-ci ne doivent être imprimés ou autrement reproduits sans son autorisation.
In compliance with the Canadian Privacy Act some supporting forms may have been removed from this thesis.

While these forms may be included in the document page count, their removal does not represent any loss of content from the thesis.
Conformément à la loi canadienne sur la protection de la vie privée, quelques formulaires secondaires ont été enlevés de cette thèse.

Bien que ces formulaires aient inclus dans la pagination, il n'y aura aucun contenu manquant. 


\section{THESIS ABSTRACT}

The architectural model is the architect's ultimate tool of representation, serving to communicate formal ideas in a three dimensional mode. The interest of this thesis lies beyond the traditional definition of the architectural model and seeks to investigate the generative potential of the "architectural model" where the Macro(cosm), the whole condition is informed by its parts, the Micro(cosm).

The architectural model as ideological communication evolved from the theory and tradition of the "analytique" drawing; investigating the idea of a graphic representation as a cognitive communicator and as a generator of architectural creativity. The threedimensional model however will respond to the limitations associated with the analytique's two-dimensional graphic technique and its inseparable bond to the surface on which it resides. While this type of drawing can be interpreted through its thoughtful play with scale and composition, it cannot break free from its plane of existence. Conversely, this thesis will pursue a generative model that allows for many, if not unlimited possibilities of interpretation granted by its three-dimensionality. The premise of these descriptive and informative models contemplates the traditions of true materiality and craftsmanship. One will be confronted with issues of materiality joints and junctions, scales, spaces and surfaces and will be required to critically negotiate these elements in order to articulate a coherent architectural proposition. 


\section{TABLE OF CONTENTS}

Thesis intent

Model - The Word 6

Historical Overview $\quad 8$

$\begin{array}{ll}\text { Program } & 22\end{array}$

$\begin{array}{ll}\text { Contextual Analysis } & 23\end{array}$

$\begin{array}{ll}\text { Site Analysis } & 26\end{array}$

$\begin{array}{ll}\text { Process } & 29\end{array}$

Process Logbook 38

Translation from Models to Architecture 72

Method of Graphic Presentation $\quad 83$

$\begin{array}{lr}\text { Conclusion } & 88\end{array}$

$\begin{array}{ll}\text { Bibliography } & 91\end{array}$

$\begin{array}{ll}\text { Appendix } & 93\end{array}$ 


\section{LIST OF FIGURES}

Fig. 1: Giovanni Battista Piranesi, The Magnificenza of Roman architecture,

Fig. 2: Marco Frascari, Analtytique Drawing, Banco Populare di Verona

Fig. 3: the Mastabe, www.legolas.org

Fig. 4: the Ziggurat, www.znanje.org

Fig. 5: Egyptian Wooden Models, Architectural Model as Machines, pg. 7

Fig. 6: Egyptian Wooden Models, Architectural Model as Machines, pg. 8

Fig. 7: Paradeigma, www.jstor.org/jstor/gifcvtdir/ap003319/04a00240_1.2.jpg

Fig. 8: Greek Paradeigma, Architectural Model as Machines, p. 10

Fig. 9: Helepolis, www.allposters.com

Fig. 10: Michelangelo presenting to the Pope, Architectural Model as Machines, p. 17

Fig. 11: Proposed site location. P. Bisson

Fig. 12: Proposed site location. P. Bisson

Fig. 13: Context of site location. P. Bisson

Fig. 14: Andre Breton, http://famouspoetsandpoems.com/poets/andre_breton/photo

Fig. 15: Book cover, Nadja, Andre Breton

Fig. 16: Max Ersnt, Max Ernst - Life and Work, p. 140

Fig. 17: Ainsi Font... Elephants. Cathedrales, Max Ernst - Life and Work, p. 114

Fig. 18: Prouns series 1, El lissitzky. http://siteimages.guggenheim.org

Fig. 19: Prouns series 2, El lissitzky. http://siteimages.guggenheim.org

Fig. 20: Model-A Elevation, P. Bisson

Fig. 21: Model-B, P. Bisson

Fig. 22: Model-B, P. Bisson

Fig. 23: Model-B, P. Bisson

Fig. 24: Model-B Logbook caption, P. Bisson

Fig. 25: Model-B, P. Bisson

Fig. 26: Model-C Elevations, P. Bisson

Fig. 27: Model-C Logbook caption, P. Bisson

Fig. 28: Model-C, P. Bisson

Fig. 29: Model-C, P. Bisson

Fig. 30: Model-C, P. Bisson

Fig. 31: Model-D Elevations, P. Bisson

Fig. 32: Model-D, P. Bisson

Fig. 33: Model-D, P. Bisson

Fig. 34: Model-E Elevations, P. Bisson

Fig. 35: Model-E, P. Bisson

Fig. 36: Model-E, P. Bisson 
Fig. 37 Model-E, P. Bisson

Fig. 38 Model-E Logbook Caption, P. Bisson

Fig. 39: Model-F Elevations, P. Bisson

Fig. 40: Model-F, P. Bisson

Fig. 41: Model-F, P. Bisson

Fig. 42: Model-F, P. Bisson

Fig. 43: Model-F, P. Bisson

Fig. 44: Initial Axonometric Study, P. Bisson

Fig. 45: Interpretation of Drawing - Window Leaver, P. Bisson

Fig. 46: Presentation Model, view from Bank Street, P. Bisson

Fig. 47: Presentation Model, Birds eyeview, P. Bisson

Fig. 48: Presentation Model, Front elevation, P. Bisson

Fig. 49: Presentation Model, Rear Elevation, P. Bisson

Fig. 50: Presentation Model, Sectional view of model, P. Bisson

Fig. 51: Presentation Model, Night condition, P. Bisson

Fig. 52: Interpretation of Drawing - Window Leaver, P. Bisson

Fig. 53: Book shelf for sister in-law, Carlo Scarpa, The Craft of Architecture, p. 24

Fig. 54: Details of poles, joints and drill holes, Carlo Scarpa, The Craft of Architecture, p. 59

Fig. 55: Baluster details, Le Architetture Di Ridolfi e Frankl, p. 255

Fig. 56: Windowsill details, Le Architetture Di Ridolfi e Frankl, p. 257

\section{Appendix}

Fig. 57: Architectural Drawings, Page 1, P. Bisson

Fig. 58: Architectural Drawings, Page 2, P. Bisson

Fig. 59: Architectural Drawings, Page 3, P. Bisson

Fig. 60: Architectural Drawings, Page 4, P. Bisson

Fig. 61: Architectural Drawings, Page 5, P. Bisson

Fig. 62: Architectural Drawings, Page 6, P. Bisson 


\section{- Crafting Matter-Reality -}

The architectural model is the architect's ultimate tool of representation. Over centuries and throughout numerous cultures, the scale model has served to communicate formal, social, cultural, and religious ideas. However, the focus of this thesis lies beyond the traditional definition of the architectural scale model and seeks to investigate the generative potential of the 'architectural model' where the Macro(cosm), the whole condition is informed by its parts, the Micro(cosm).

The architectural model as an ideological communicative device evolved from the theory and tradition of a graphic method practiced throughout the Beaux Arts period, the "analytique" drawing; the creation of a synoptical graphic representation, as a cognitive communicator and generator of architectural creativity. The "analytique" drawing is a very specific way to study the role of the detail as a generator through graphic means: "In this graphic representation of a designed or surveyed building the details play a predominant role. They (analytique drawings) are composed in different scales in the attempt to single out the dialogue among the parts in the making of the text of the building. Sometimes the building as a whole is present in the drawing, and generally is represented on a miniscule scale, and so it seems a detail among details."1

\footnotetext{
${ }^{1}$ Marco Frascari, The Tell-The-Tale Detail, p 24.
} 
The source of the "Analytique" and its role in the interpretation of architecture can be traced back to the technique of graphic representation and composition developed by Giovanni Battista Piranesi, in his etchings surveying the Magnificenza of Roman architecture. (fig. 1)

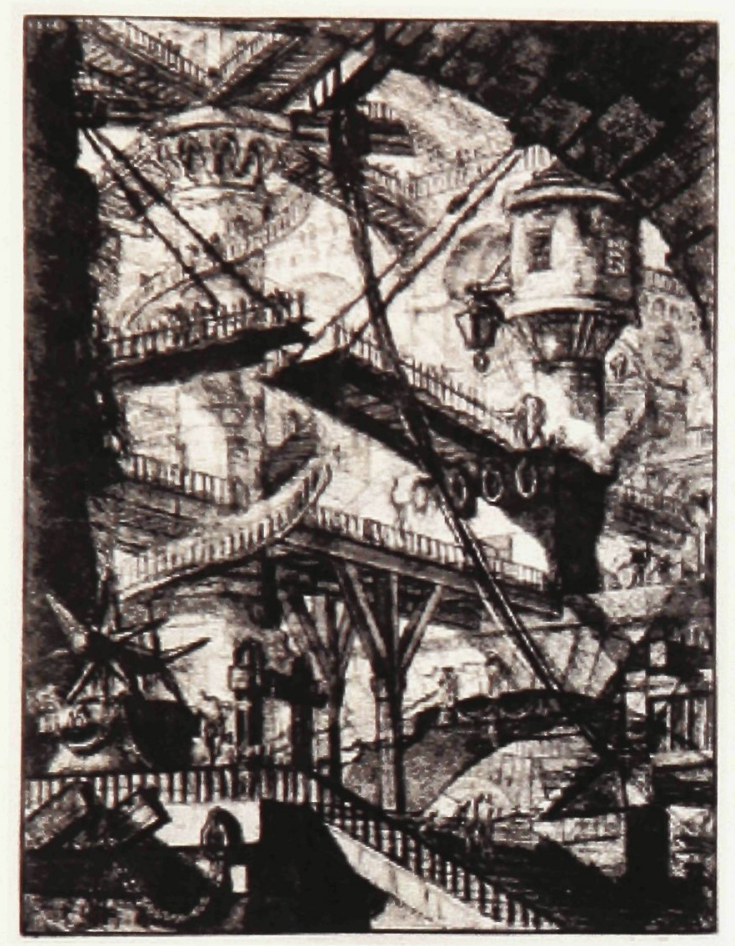

Another example of this graphic technique can be found in the drawings of Marco Frascari His successful attempt at re-interpreting the Banco Populare Di Verona by Carlo Scarpa, through the graphic technique of the 'analytique' is a clear example of the potential playfulness of the composition and the multiple scales of the building's representation. 
(fig.2).

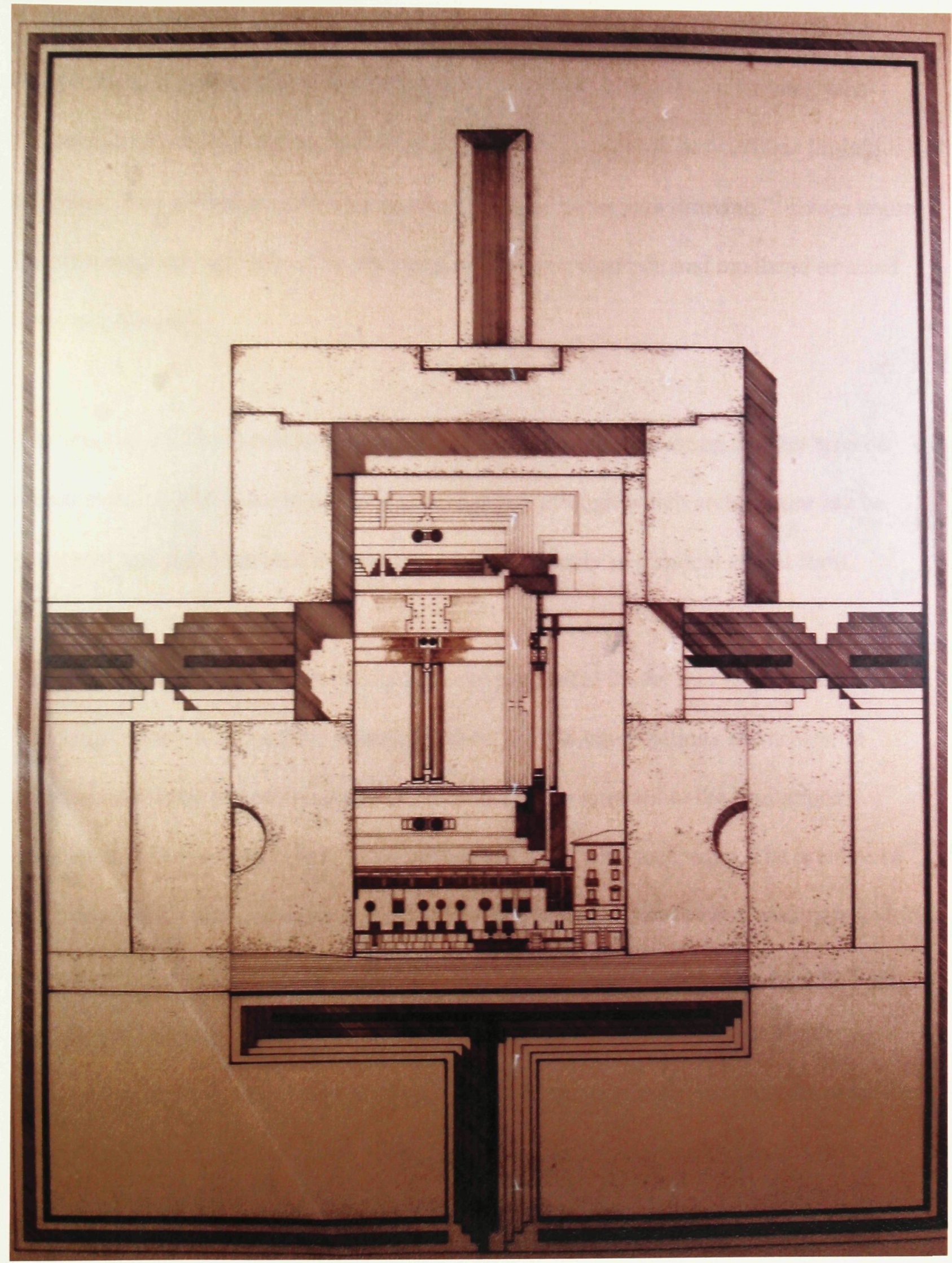


While the 'analytique' can be interpreted through its thoughtful play with scale and composition, it is inseparable from the surface on which it resides. In Robin Evans' Translation from Drawing to Buildings, he states, "the drawing has intrinsic limitations of reference. Not all things architectural can be arrived at through drawing." ${ }^{2}$ Evans argues that drawings can sometimes be disengaging, oblique, abstract, and mediated or acted upon at a distance.

The objective of the thesis is to investigate the possibility of creating another type of architectural model that can become a mechanism through which architecture can be generated and demonstrated in three dimensions; namely in physical model form.

Conversely, this thesis will pursue a generative physical model that allows multiple "readings" granted by its three-dimensionality, playful compositions and scales. In addition, the three-dimensional models will inherently respond to the limitations associated with the "analytique's" two-dimensional graphic technique. The premise of these descriptive and informative models contemplates the traditions of materials and true craftsmanship. Furthermore, physical modeling as a means of generating architecture, engages corporeal properties that drawing neglects. Modeling allows for direct involvement by both author and viewer; it engages the substantial and tangible qualities belonging to this particular mode of investigation and they require presence and immediacy. Through modeling, these corporeal properties will help to resolve issues of

${ }^{2}$ Robin Evans, Translations from Drawing to Buildings and Other Essays, p 159. 
materiality, joints, junctions, scales, special relationships, and surfaces. It will be necessary to critically negotiate and translate these elements, and spatial moments, in order to articulate a coherent architectural proposition. 


\section{'MODEL', THE WORD}

Before we do a brief historical overview of the scale model, it is necessary that we comprehend the meaning of this loaded term. Model is a term that carries multiple meanings and is frequently used in sentences without any care for its etymological roots. In the present context, 'Model' is employed with little or no regard to its origins or meaning which is why we must seek its original definition in order to contemplate a model as a generative tool.

The etymology of the word "Model is borrowed from Middle French 'modele', from Italian modelle, a model or mold, from the vulgar Latin 'Modellus'. 'Modellus' is a diminutive of the Latin modulus, a diminutive of modus, which means to measure." ${ }^{3}$ Model, in this context, is considered a basis of measure, of reference. However, in the context of architecture, the French term 'maquette' is probably the closest to the concept of what this study refers to as the architectural model.

If we elaborate the source of 'maquette' further, we can follow its roots to the Italian term 'Macchietta', which can be defined as the physical act of redefining a blotch of ink. More significantly though, 'Maquette' is also known to be a rough sketch of a small theatrical design. Primarily, " "Maquette" is a drawing that gauges the general appearance or composition of a theatrical setting. The key to the significance of "Maquette" is its relationship to the concept of demonstration. In addition, the word "demonstrate" comes

${ }^{3}$ Albert C Smith, Architectural Models as Machines, p 62. 


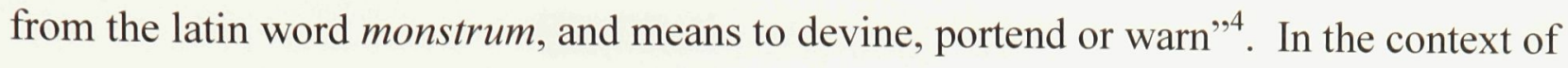
the divine entity, "a demonstration offers a foreshadowing of coming events and allows a certain prophetic indicator of meaning through marvel, prodigy and wonder. ${ }^{.5}$ In other words, demonstration embodies the projection of an idea.

The etymological root of the word 'demonstrate' is carefully defined in Marco Frescari's insightful book: Monsters of Architecture. The etymological definition of demonstration can be broken down as such: "de" which means entirely, and "monstrare" is to point out, or to show. It stems from Latin "monstrum". In respect to the definition of 'demonstrare', Frascari defines the role of the architect as the one who demonstrates "through tangible signs the intangible that operates in the tangible."

From the roots of the word, comes the evolution of the word model throughout history. Contrary to popular belief, models were not just used for design purposes, but were also considered to be a means to understanding a culture's universe. The next chapter is a chronological overview of the evolution of the scale model throughout history.

\footnotetext{
${ }^{4}$ Marco Frascari, Monsters of Architecture, p.2

${ }^{5}$ Marco Frascari, Monsters of Architecture, p.2

${ }^{6}$ Marco Frascari, Monsters of Architecture, p.3
} 


\section{HISTORICAL OVERVIEW}

This historical review draws attention to significant relationships occurring between the social and cultural context and understanding the role of architectural models within these dynamic conditions. Ancient Egyptians present a well-documented and historically logical beginning point for an historical overview on the architectural model and its origins.

The ancient Egyptians strongly believed in the afterlife. The notion of the afterlife was a predominant feature in Egyptian beliefs. The significance of the afterlife for Egyptians was evident when we consider the wide range of hieroglyphs and artifacts collected from their civilization. The ideology of the afterlife was also present through other forms. If we consider the relationship in the graphic representation of both the house and tomb in Egyptian hieroglyphs, the similarity between both are striking. The similarity occurs because they share a reciprocal relationship in their meanings. Furthermore, collections of small handcrafted models, recovered from tombs, allow us to appreciate their fascination with the afterlife. In fact, Egyptians went through great lengths "toward preparing their tombs with all the equipment and provisions necessary to keep them at least as happy in death as they were in this life." ${ }^{7}$ The importance of these Egyptian models is significantly more important when we consider its relationship to the Egyptian cultural beliefs. These models were found in all types of tombs such as pyramids, Mastabe's (fig. 3) and Ziggurat's (fig. 4).

${ }^{7}$ Smith, Albert C, Architectural Models as Machines, pg.6 
Fig. 3 Mastabe

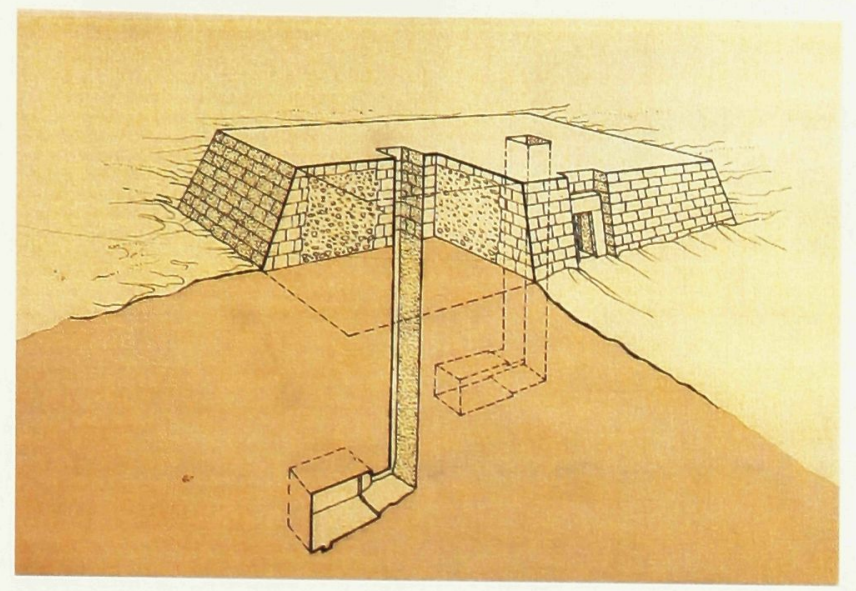

Fig. 4 Ziggurat

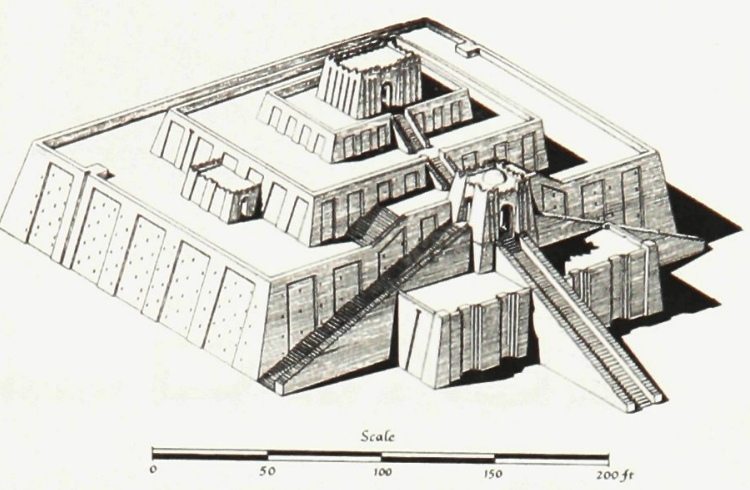

It was from the era of Imhoptep, the great Egyptian architect, that archeologist uncovered the majority of these insightful models. Inside these pyramids and tombs, discoveries of hundreds of small, carefully hand crafted wooden models, with varying characteristics, were documented and preserved.

The needs of the deceased would be magically supplied eternally through smallscale model butcher's shop, bakeries, granaries, carpenter's workshop, and model boats. Egyptian small-scale models were built so that the deceased would enjoy a continuous supply of food and the company and support of family and servants. They ensured that the deceased would maintain his or her proper status in the next world, exempt from the duties of manual labor. ${ }^{8}$

These models embodied much more than just a physical representation of amenities; they played an integral role to a successful spiritual transition, and prosperous afterlife.

(fig.5)

${ }^{8}$ Smith, Albert C, Architectural Models as Machines, pg.7 


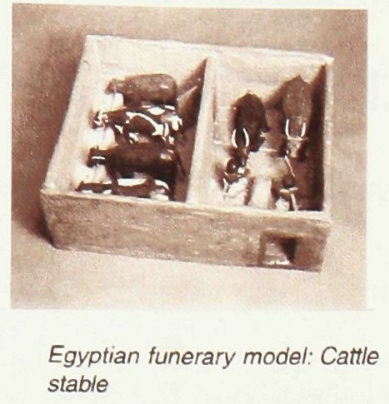

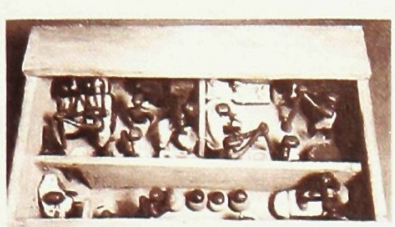

Egyptian funerary model: Bakery

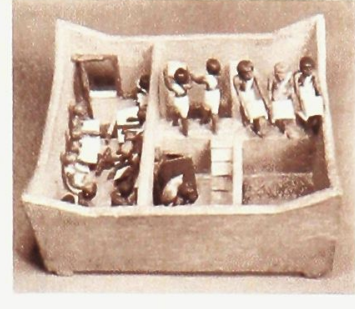

Egyptian funerary model Granary shop

These models provided the necessary link between both the spiritual realm and the physical realm. In the context of Egyptian culture, these models ensured continuous prosperity to those who passed away granting them success and wealth in the afterlife. For us, these models represent a window through which we can perhaps seize an instant of their cultural beliefs.

(fig.6)

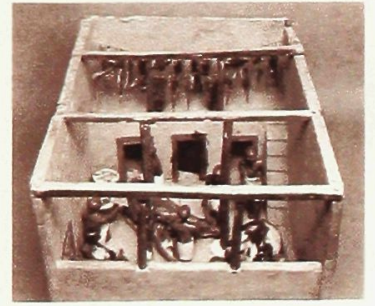

Egyptian funerary model: Butcher shop

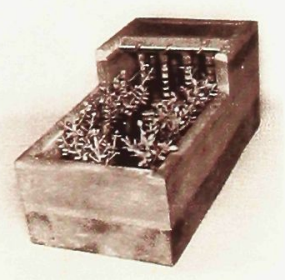

Egyptian funerary model:

As we proceed in this attempt to retrace the origin of the model, we come to a civilization which has made a profound influence in our western way of thinking. Classical Greece, also referred to as the Golden Age of Athens, was the first democratic society dedicated to "human excellence in mind and body, to philosophy and to the arts and sciences"9. Their determination for excellence as a society had a tremendous impact on their architecture. Through geometry and form, ancient Greece established standards in the form of iconic

\footnotetext{
9 Albert C. Smith, Architectural Models as Machines, p.9
} 
details and proportions. To this day, these standards of proportion and form are greatly valued in contemporary architectural theory. Greek architects did not use conventional scale models to represent their architecture, instead they would use models of a different nature; models that were deeply rooted in mathematics and geometry which in turn were reinterpreted and elaborated through their architectural language.

The Greeks did however discover another type of architectural model. They created a type of model that became crucial in the development of Greek architecture. This model embodied the characteristics of a prototype, which was known as: the paradeigma. (fig. 7)

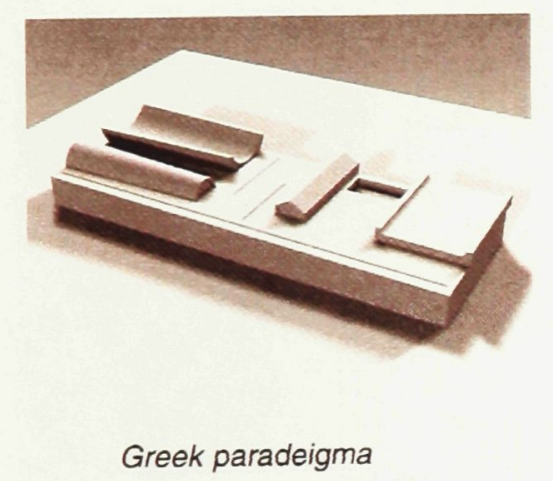

The paradeigma "is a specimen or an example used to study specific architectural elements, such as triglyphs or capitals which required a three-dimensional design, and in cases where carved or painted decoration has to be shown." ${ }^{10}$ These models were generally on a scale of one to one and were fabricated of the materials in question in order to get an accurate evaluation of the specimen.

${ }^{10}$ Albert C. Smith, Architectural Models as Machines, p.10 
The significance of these models as prototypes explains a great deal about their architectural heritage along with a fascination for sophisticated detailing. The models that they produced were not conceptual in nature because Greek architects sourced their concept on historically pre-established architecture. "Greek architects generally used already well-defined concepts of explaining invisible things and mainly dealt with the refinement of details"11. Thus the purpose of the "paradeigma" was to evolve the architectural language of Greek architecture, which consequently propelled Greek craft into a higher level of detailing, architecturally symbolizing their socio-cultural status as an elite civilization. (fig. 8)
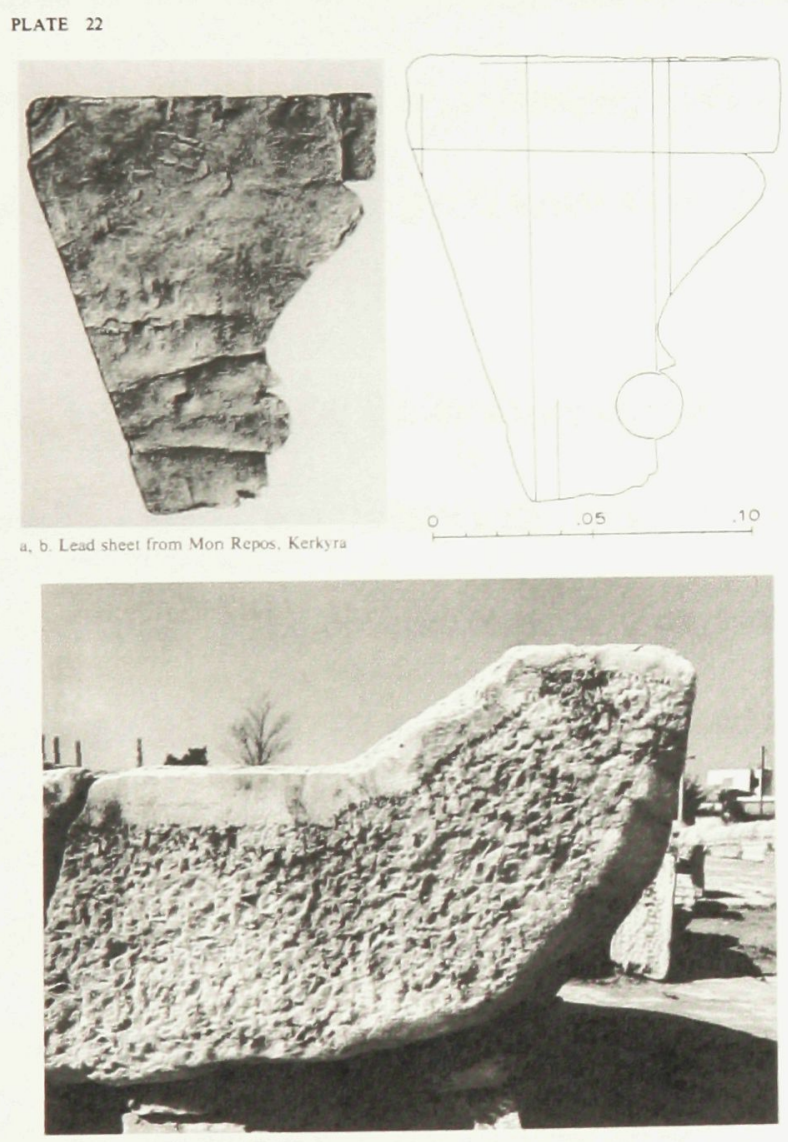

c. Crowning molding from Eleusis

JOHN TRAVLOS: TAPASEITMA

${ }^{11}$ Albert C. Smith, Architectural Models as Machines, p.11 
The "paradeigma" took on a very prominent role in the process of defining Greek architecture. Without these models and their geometrical notions and rules of proportions, the architectural language of Greek culture may have been significantly different. The role of the architect in this instant was to gather such models. Whether he, the architect, was the one to actually build it is another issue. At the very least, the architect's responsibility was to determine the dimensions and proportions of the "paradeigma". This fundamental relationship between the craftsman's and the architect was crucial in the development of Greek architecture. The knowledgeable craftsman had the responsibility to operate within the parameters set out by the architect. Although the architect had control over the project as a whole, managing scale and proportion, it was the skillful craftsman who seemed to have profound knowledge of the architecture's soul.

It was only during the Roman Empire that the architectural model was utilized, as we typically understand them today. Generally speaking, Roman architecture was mostly derived from Greek architecture. In fact, many architects from the Roman Empire were known to be Greek. The source of the Iconic, Ionic, Doric and Corinthian orders were also from the classical Greek era but were later reinterpreted with a higher level of craftsmanship by the Romans. The Roman Empire made their mark on architectural history through the development of concrete and innovative use of brick masonry. This new material provided the Romans with a unique opportunity to create architectural masterpieces such as the pantheon and the coliseum. 
In relationship to the architectural model, it is important to reflect on this question: How does one convince an empire to undertake such feats as the coliseum? In his tenth book, Vitruvius writes an insightful anecdote allowing us to grasp the role of small-scale models with respect to the Roman Empire. He writes,

For Diognetus was a Rhodian architect, to whom, as an honour, was granted out of the public treasury a fixed annual payment commensurate with the dignity of his art. At this time an architect from Aradus, Callias by name, coming to Rhodes, gave a public lecture, and showed a model of a wall, over which he set a machine on a revolving crane with which he seized on Helepolis as it approached the fortifications, and brought it inside the wall. The Rhodians, when they had seen this model, filled with admiration, took from Diognetus the yearly grant and transferred this honor to Callias.$^{12}$ (fig.9)

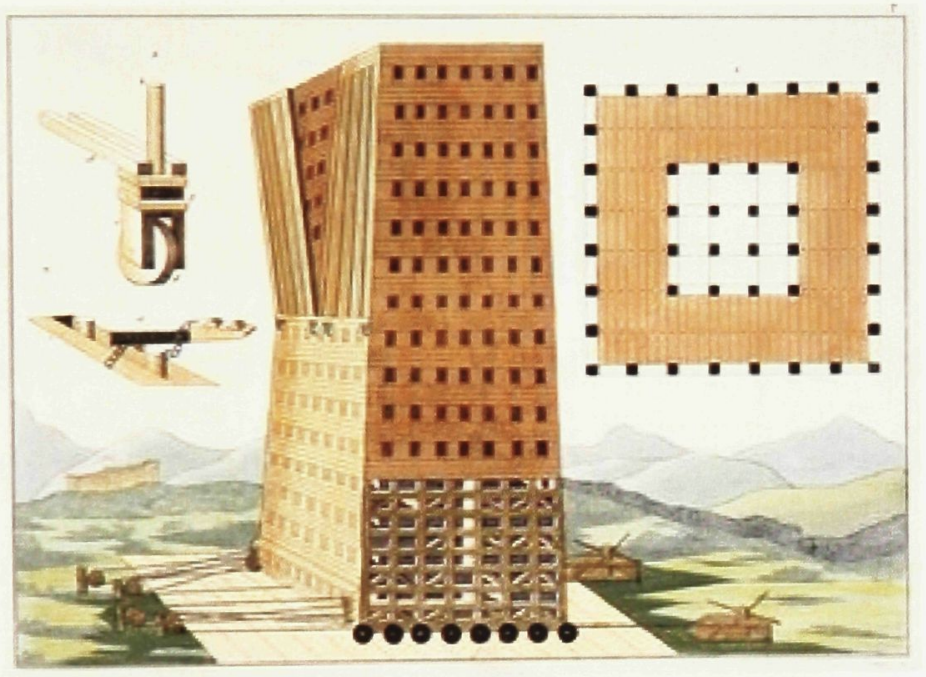

Through this anecdote, we can begin to understand the context in which the scaled models came to be during the roman period. They had discovered the persuasive potential granted by the scale models causing at once disbelief and admiration amongst the population. The power to convince was the power to conquer.

12 Albert C. Smith, Architectural Models as Machines, p.14 
Furthermore, the anecdote raises fundamental issues about architectural models that could have very well affected the role of the model in architecture to this day.

The Romans seemed to be well aware of the persuasive application of the smallscale model. Second, the small-scaled model built by Callias permitted a population untrained in architecture to easily view the possibilities of a full-scale mechanism. Third, the roman small-scale model presented a mechanism granting the architect and the population an opportunity to perceive a possible future. ${ }^{13}$

The use of models in this sense is extremely similar to the purpose of our present models. Historically the Romans must have been the first to use the model as a communicative device in order to represent ideas in all their aspects to a general and untrained population.

This new method of articulating architectural concepts through modeling needed a new educational framework based on multiple disciplines and arts. "Vitruvius believed that an architect should be a man of letters, a skilful draftsman, a mathematician, familiar with historical studies, a diligent student of philosophy, acquainted with music; not ignorant of medicine, learned in the response of jurisconsult, familiar with astronomical calculations." ${ }^{14}$ This curriculum was believed to be the new academic model of architectural education. In light of this new spectrum of responsibilities, architects would now possess a profound foundation of knowledge to challenge such architectural concepts.

13 Albert C. Smith, Architectural Models as Machines, p.15

${ }^{14}$ Albert C. Smith, Architectural Models as Machines, p.16 
After the fall of the Roman Empire, Christianity became the dominating religion of the empire. This crucial change allowed the church to become the ruling authority over Europe. The shift in power initiated a social and cultural transformation of European philosophy, science, literature, art, and architecture. Consequently, the church as a governing body significantly affected the framework of architectural education. Within the new curriculum, stated in the treatise of geometer Papus of Alexandria, the education consisted "of geometry, arithmetic, astronomy, and physics, and a manual part that involved work in metal, construction, carpentering, and the art of painting, and the practical execution of these matters". ${ }^{15}$ One of the major components omitted by this new educational structure was that of philosophy and theory. The absence of philosophy in architectural education was in the church's interest in order to control the intellectual growth of architects. This new curriculum was finely crafted by the church in order to eliminate the potential of alternative philosophical views of the world. "The person who mastered the curriculum became a "mechanic" (or mechanikos), a term applied to a number of Byzantine architects." 16

"The concept of architects as mechanics illuminates the relationship of the architects to their scale model. There are two types of mechanics: those who are skilled in the creation of machines, and those who are skilled in maintaining machines." ${ }^{17}$ When we consider that the "church operated as a mechanism by which to demonstrate the word of God" $"$, it

\footnotetext{
${ }^{15}$ Albert C. Smith, Architectural Models as Machines, p.18

${ }^{16}$ Albert C. Smith, Architectural Models as Machines, p.19

17 Albert C. Smith, Architectural Models as Machines, p.19

${ }^{18}$ Albert C. Smith, Architectural Models as Machines, p.20
} 
is clear that the role of the architect as a creator was being replaced with the role of maintaining. The architect was no longer required to interpret the "house of God", but rather, the act of creating was revoked and substituted by a pre-established image created by the church. Essentially the architecture was already well designed. The architect's role was simply to carry out the mechanical processes that allowed the architecture to materialize under the scrutiny of the church. The church intended to spread a specific message by employing the Gothic cathedral as "mechanisms used for demonstrating the Christian religion. The building is designed to enhance an awareness of the presence of God and to carefully control the relationship of the parishioners to the church hierarchy." 19 (fig.10)

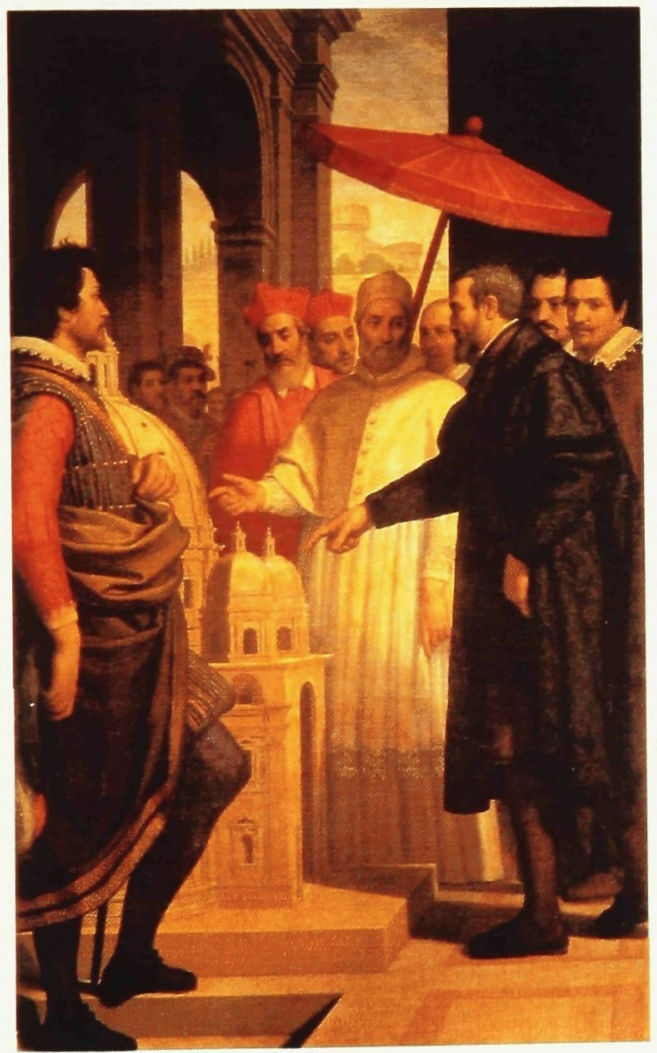

If we consider the scale model as a communicative tool of a formalized idea, then it is possible to interpret the gothic church as a model that communicates the ideals of Christian religion in built form. In this case, the model was not used to communicate an architectural idea but rather, something much more elaborate and complex: Christianity. Throughout the middle ages, it was necessary to promulgate this religion to all people through these built mechanisms known as churches. Gothic churches would commonly depict the story of medieval Christianity

${ }^{19}$ Albert C. Smith, Architectural Models as Machines, p.20 
through sculptures or stained glass windows to reach out to an illiterate population. This successful device allowed the Christian religion to become an overwhelming power reaching across many continents.

The Renaissance is a very critical period in relationship to the architectural scale model and its role within the architectural profession. This period brought forth different types of models and modeling techniques that were transmitted from Roman practices. The scale model was now playing an integral role to the making of architecture and becoming the preferred tool of communication by architects and "served the Renaissance builders when it came to the execution of a building."

We cannot discuss the Renaissance period without referring to Italian architects and architecture. Italian architect, Leon Batista Alberti, lived in the beginning of the Renaissance and later became a central figure in relation to architectural theory. Multiple subjects fascinated Alberti such as architecture, painting, music, mathematics, and athletics. "Architecture was a somewhat theoretical pursuit for Alberti. Not faced with the detailed or pragmatic problems of building, he devoted himself to demonstrating principles of proportion and codification for reuse of classical building parts. ${ }^{20}$ In his architectural treatise, On the Art of Building in Ten Books, Alberti specifically addresses the use of scale models.

${ }^{20}$ Albert C. Smith, Architectural Models as Machines, p.26 
The major difference during the Renaissance is that the architects regained their authority over their profession granting them creativity and interpretation. This change was initiated "by the changing philosophical standards of the period. Ideas developed through scale models were not as strictly controlled by the organization of the church or guilds as they had been during the Gothic period."21

It is important to emphasize that the small-scale model had now become a crucial part of conveying necessary information to those executing the design. It was truly employed as a mechanism that projected architectural intent. Furthermore, the model served as a device to foresee the future; more precisely, it helped to identify likely troubles and expenses, it helped to quantify parts and pieces, and it also allowed architects to show their 'clients' the future in a three-dimensional fashion. Alberti explains,

Having constructed these models, it will be possible to examine clearly and consider thoroughly the relationships between the site and the surrounding district, the shape of the area and the number and order of the parts of a building, the appearance of the wall, the strength of the covering, and in short the design and construction of all the elements discussed in the previous book. ${ }^{22}$

The way in which Alberti describes this rigorous use of models as a means of crossreferencing ideas, to ensure coherence throughout all the parts, describes a logical process by which ideas evolve and problems are resolved. This analytical modeling process has the potential to achieve Alberti's theory of 'Concinnitas'; he saw architecture as the art of selecting appropriate details which resulted in beauty. Beauty, as Alberti defines it, is the

${ }^{21}$ Albert C. Smith, Architectural Models as Machines, p.26

22 Albert C. Smith, Architectural Models as Machines, p. 27 
"concinnity of all the details in the unity to which they belong; in other words, beauty is the skillful joining of parts by a normative by which nothing can be added, subtracted, or altered for the worse." ${ }^{23}$ According to Alberti, beauty is an orchestration of precise relationships between the detail and the attached meaning. Consequently, beauty is the product of a specific process of signification, and "concinnity is the process for achieving $i t^{\prime \prime}$.

Concinnity is the correspondence of three basic requirements: 1-Numeratio (numbers) 2Finitio (finishing) 3-Collocatio (composition). ${ }^{24}$ The strength of this theory however, lies in the overlapping relationships shared by all three components, which are essential in the making of 'beautiful' architecture. The numbering, which is typical expressed though geometry, is the fundamental organizational system by which the meanings and mathematical correlations are derived. The finishing is informed by the later and can't determine a proportional system without a numeral basis. As for the collocation, it remains dependent on both numbering and finishing as a source of information to allow the successful manipulation of the detail by means of scale as an ordering tool.

Within the context of 'concinnitas', the role of the architectural model as a mechanism of resolution, becomes clear. The model is not only a tool used to present the idea of a building, but it also generates a process that inherently strengthens an idea.

Using scale models examines every part of your proposal two, three, four, seven, up to ten times, taking breaks in between, until from the very roots to the uppermost tile there is nothing, concealed or open, large or small, for which you

23 Marco Frascari, The Tell-The-Tale Detail, Via 7, (1984), p. 26

${ }^{24}$ Marco Frascari, The Tell-The-Tale Detail, Via 7, (1984), p. 27 
have not thought out, resolved, and determined, thoroughly and at a length, the most handsome and effective position, order and number. ${ }^{25}$

The socio-cultural relationships that are defined in the historical overview, between scale models and their historical context, help to substantiate the reasoning behind the premise of this thesis. The historical overview considers the relationship and the role of the model to the influential conditions of its era. Also, the examples of scale models presented herein are a response to the underlying socio-cultural forces described during several key historical periods. Similarly, the architectural models that this thesis seeks to generate architectural creativity, creates possibilities, and solicits multiple interpretations is, to a certain extent, are a reaction to the socio-cultural conditions under which we presently operate.

${ }^{25}$ Albert C. Smith, Architectural Models as Machines, p. 29 


\section{PROGRAM}

The program chosen for this thesis is a Pastry store. This program was chosen because it shares relationships to architecture and the crafting of models. Similar to architecture, the culinary art of pastry making is highly aware of the importance of artistry and composition in respect to the creation of delicacies. In true tradition, irrelevant of size or complexity, there is a passion to capture a moment of supreme taste in all of the edible creations. In the realm of pastries, craft is a crucial aspect in the presentation of these palatable models. The appropriate modes of display for the varying types of pastries are carefully considered. Pastries embody the desire for the absolute, the sublime - a moment of perfection. Architects and pastry chefs alike have mastered the use of fundamental materials or ingredients associated to their profession. Mastering these basic materials allows them to improvise and transform these materials or ingredients into marvelous creations. The site chosen for this particular program is located in Ottawa, Ontario, Canada. More specifically, it is a small site within a trendy central neighborhood called the Glebe. 


\section{CONTEXTUAL ANALYSIS}

The Glebe - Ottawa's first suburb, was annexed by the city in 1889 . The area is called the Glebe because in the initial 1837 survey of Ottawa the area was allocated to St. Andrew's Presbyterian Church. The word "glebe" means church lands and the area was originally known as "the glebe lands of St. Andrew's Presbyterian Church". When the area was opened for development in 1870 , real estate agents began to refer to it simply as "The Glebe". The area is strongly defined by the Queensway (formally the railroad) to the north, and Lansdowne Park to the south. Other prominent geographic characteristics include the natural and artificial water boundaries located in areas such as Patterson's Creek, the Rideau Canal and Brown's Inlet.

In 1900, the Ottawa Electric Street Railway was established, with one of its first routes running south along Bank Street. This allowed workers to live in the Glebe and take the streetcar to work. Most Glebe houses date from this era, and it became home to many of the working class. Since the area was originally designated as church land, its intended use went through many different programmatic changes such as market and exhibition grounds. It eventually became home to mostly Anglophone, Protestant civil servants who could afford to take the streetcar to work downtown or on Parliament Hill and had the leisure time for strolls and canoeing. 
In the middle part of the century the Glebe changed as the middle class moved to more distant suburbs such as Alta Vista and Nepean and the Glebe became transformed into a predominantly working-class neighbourhood with the houses subdivided into multiple apartments. The area underwent significant gentrification in the 70s, including a successful anti-traffic movement supported by Canada's first woman mayor, and Glebe resident, Charlotte Whitton. It continues to have a distinct sense of solidarity and community involvement, including a strong tradition in public education and diverse local events that attracts thousands of visitors to the neighbourhood.

The Glebe has been able to maintain its many centennial homes that compose the character of this historically rooted community. Throughout the years however, the neighbourhood was transformed by a mixture of architectural styles, with roughhouses from the 1910s and a number of high-rise from the 1970s looming in the background.

The most prominent commercial street that runs through the Glebe is Bank Street. Contrary to popular belief, the street is not named after the Bank of Canada headquarters at the corner of Bank Street and Wellington Street. The street name dates back to the 19th century, whereas the bank was founded in 1934. It is believed that the road was named after the "bank" of the Ottawa River that borders its northern end and the Rideau River to the south. 
Bank Street is a retail and diversified business district, officially known as the "Bank Street Promenade". The street is dotted with common signage affixed to streetlights and street-level advertising billboards showing this distinction. There also exists a shopping district in The Glebe running exclusively along Bank Street from approximately the Queensway to Holmwood Avenue. Bank Street is home to Lansdowne Park, a sport and recreational facility and home to the Ottawa 67's play. In general, Bank Street is composed of a historic fabric ideal for pedestrian traffic. Consequently, this slows down the pace of vehicular traffic, making this street a desired area for locals. 


\section{SITE ANALYSIS}

The designated site for this thesis project is situated in the heart of the Glebe community. It is located on Bank Street, between $3^{\text {rd }}$ and $4^{\text {th }}$ avenue. In Ottawa, this area has become one of the most desired commercial and residential areas. It has attracted a wide diversity of people ranging from students to young families as well as seniors citizens. The area has become attractive to a variety of individuals for a number of reasons; it is known for its numerous independent shops and retail stores, it is dotted with many cafés, it is proximate to public transportation and to the Rideau Canal, it is also a few blocks from the Lansdowne Park where many public exhibition and sporting events take place. (fig. 11)

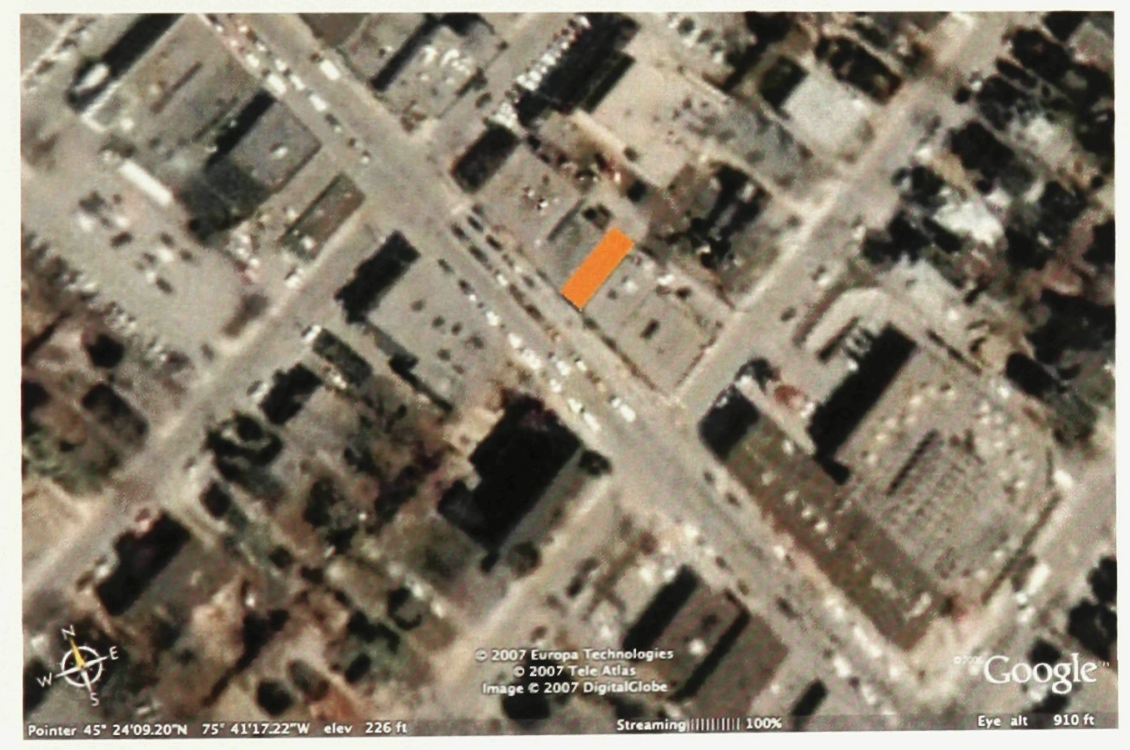

The site itself runs East to West with a western façade on Bank Street and is neighbored by two commercial establishments; Agora to the North is a one-story commercial retailer and Mrs. Tiggy Winkles, to the South is a two-story children's toy store. At the rear of the property we find a parking lot with access onto $4^{\text {th }}$ Avenue (refer to site plan). Across 
the street there is the $4^{\text {th }}$ Avenue Baptist Church, along with several shops. The site is approximately $4.5 \mathrm{~m}$ wide by $20 \mathrm{~m}$ deep. There is a three-story building height restriction in this area. (fig. 12)

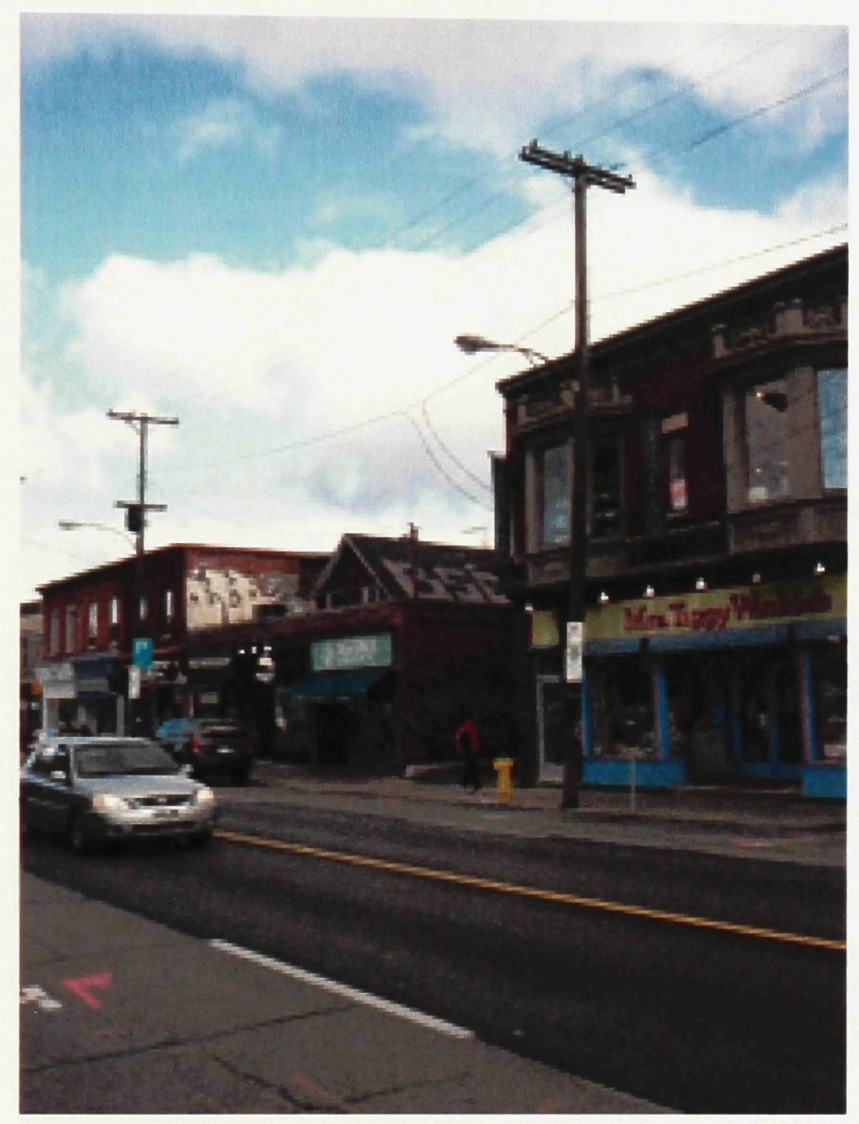

Bringing natural light into the core of the proposed architecture will be a challenge due to the inherent condition of this infill site and its already limited façade area. It's important to consider the two-story neighbouring building to the south because of its location relative to the sun's path. In any case, the proposed architecture for this site will need to respond to the light conditions that are inherently bound to the site. 
(fig.13)

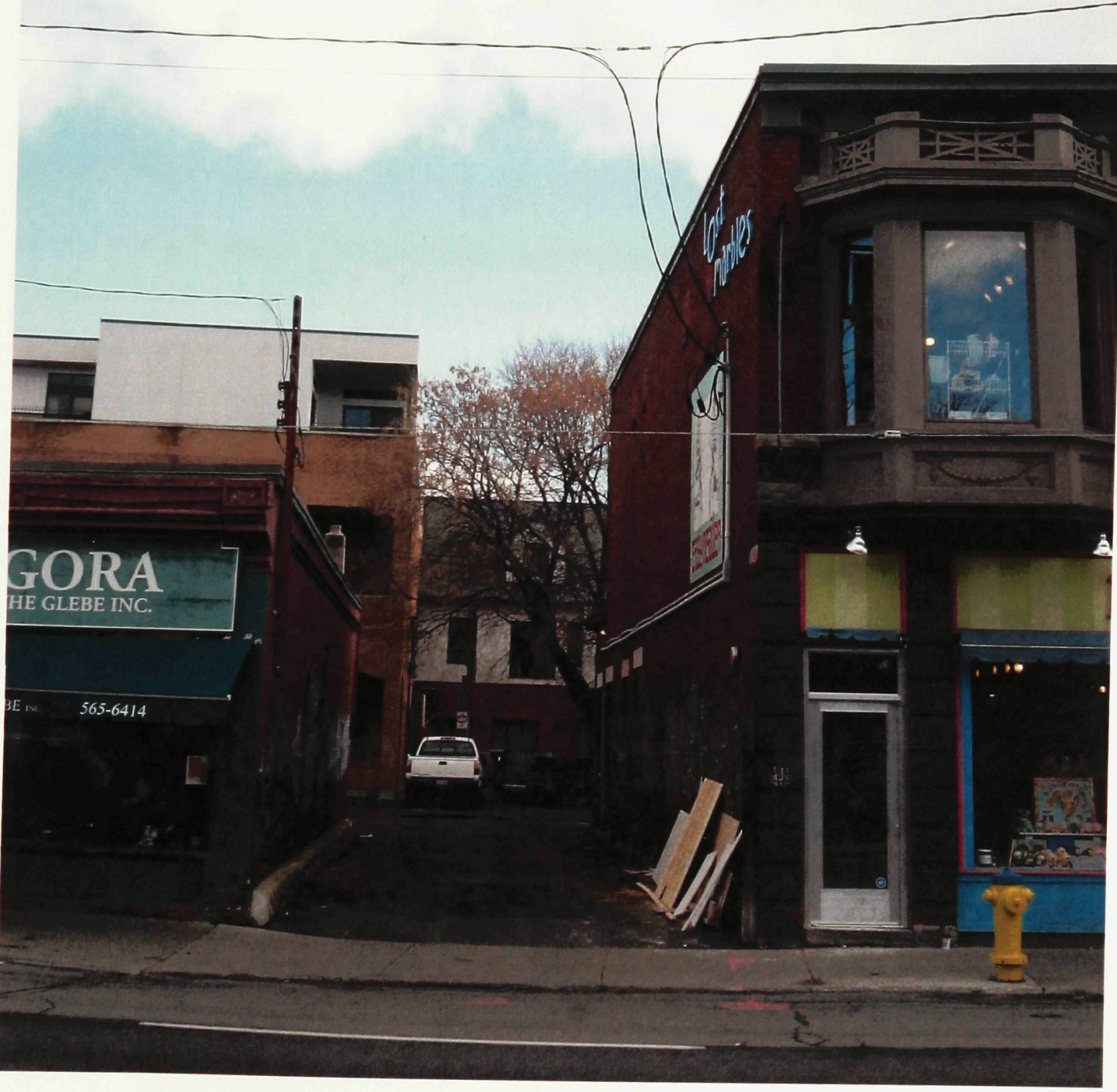




\section{PROCESS}

The interest of this process is to develop a methodology through which one can create and investigate an alternative "architectural model" as a source of architectural inspiration and creativity. The fundamental issue concerning the creation of these models is to find the appropriate approach to their development. It is crucial that the outcome of these models shall not be preconceived otherwise the model and its purpose will be flawed.

Preconceiving these models would be limiting and misleading, and would definitely not be as engaging and fruitful in nature. If the models are premeditated, it is only a means to an end, which should not be the case. One of these models could be interpreted as a manuscript for several other architectural projections.

The challenge occurs in finding an appropriate method through which the architectural models can materialize. The first model for example, developed from the composing of spatial relationship using found materials; from this experiment, it becomes apparent that the techniques of collage, and its theoretical foundation have proven beneficial in the making of a spontaneous model. It becomes necessary to elaborate the principles and pursue the methodology of collage as a precedent to these architectural models. 


\section{COLLAGE:}

The practice of collage emerged from the surrealist movement in the early 1920's. The basis of the movement was in response to the overwhelming pressure exerted by excessive rational thought and bourgeois values present in the art establishment of the time. The movement, which began in Paris, was composed of artists and writers that aimed to convey their alternative and political thoughts to the public by means of writing, painting, sculptures, public performances, and poetry. Amongst these was André Breton, poet and writer, who quickly became the most predominant figure and spokesman of the surrealist movement. (fig. 14)

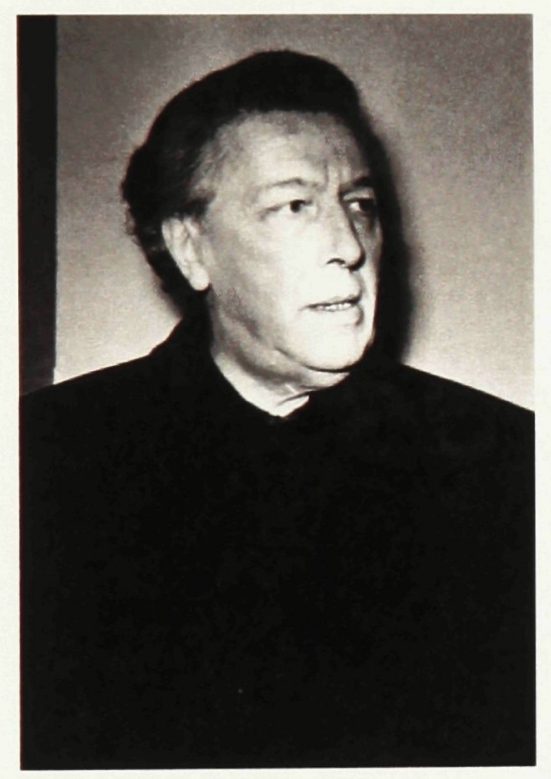

The surrealists were attracted to the notion of the automatic, the unconscious intuition if you will, and soon began to explore, through writing and painting, the potential of the automatic. They collectively published books demonstrating this alternative method of documenting experiences. These moments, ideas, dreams, or feelings were textually described as they were, as they occurred, without censuring or editing. Breton also published a novel entitled 'Nadja'. (Fig. 15) The novel, which followed the same automatic procedure, described Breton's fascination with a young woman in Paris over the
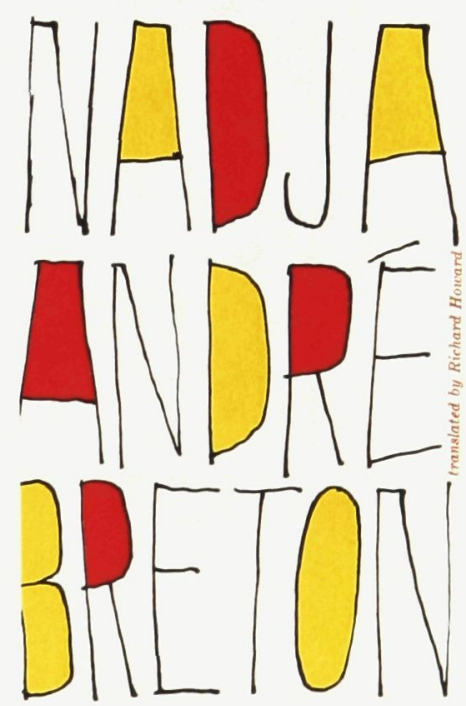
course of ten days. Automatic writing, however, was not the only means of formalizing this mode of expression.

Concurrently, collage, which became quite popular amongst the supporters of the movement, was another medium of expression that surfaced during the surrealist period.

"Collage (and by extension frottage), tapping the resources of the unconscious mind, are considered processes parallel if not equivalent to automatic writing, in their capacity to stimulate the hallucinatory powers of the artists and generate flow of multiple, contradictory images, as in hallucinations or visions of half sleep." ${ }^{26}$

The premise of this method was to develop a composition, using imagery, found objects, colour swatches, solely driven by one's own intuition, automatic and subconscious response. Many artists, such as Max Ernst, have used this technique extensively throughout this period.

Max Ernst (fig. 16) was born in Germany in 1891, near Cologne. Later, Ernst would pursue his academic interest in philosophy, but it wasn't long before he abandoned the academic institution to pursue his true passion in visual arts. Oddly enough, Ernst never received any official or academic artistic training. His artistic endeavors were harshly interrupted when he was forced to join the German

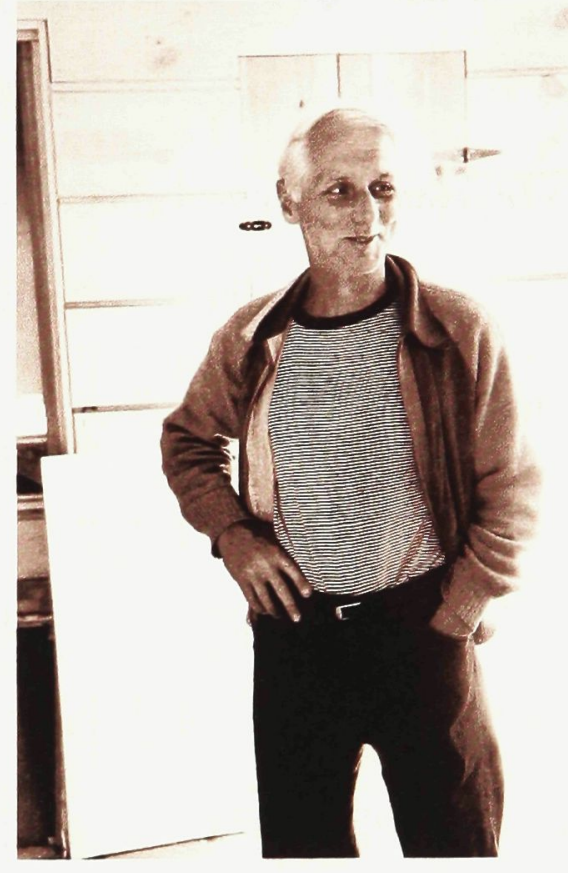

${ }^{26}$ Elza Adamowicz, Surrealist Collage in Text and Image, p. 7 
army during the First World War. Ernst states in one of his autobiographies, "Max Ernst died the $1^{\text {st }}$ of August, 1914". Although intrusive in his development as an artist, the War was a tremendous source of inspiration for his later works. In his book 'Max Ernst, Life and Work', he poses the question,

What is the technique of collage? I am tempted to see in collage the exploitation of chance - Meeting (or artificially provoking meeting) of two (or more) distant realities on an unfamiliar plane - and the flash of poetry which results from their mutual approach. ${ }^{27}$

In this passage, Ernst highlights the power that chance can have over this method of artistic creativity. Ernst also seeks to emphasize the richness of the relationship in the sometimes provoked 'meeting' of distant objects or disjointed imagery. The issue of chance addresses the initial concern about the models being preconceived. Allowing the influence of chance in relationship to the method of collage inherently eliminate any preconception. Chance is about different possibilities occurring, which would subsequently imply a choice. The act of choosing amongst different possibilities entails multiple outcomes therefore making it impossible to preconceive the end. Breton also "stresses the importance of chance in the manufacture of collage, referring to the encounter between objects 'préalablement disqualifié et tiré au hasard' (disqualified to preconditions and randomly chosen), which suggests that collage is the result of an automatic process" 28 . Furthermore, "Collage is also a material practice which deliberately subverts traditional models of representation and bourgeois value systems, through strategies of displacement and perversion. Thanks to its conscious manipulation of the

${ }^{27}$ Werner Spies, Max Ernst Life and Work, p. 54

${ }^{28}$ Elza Adamowicz, Surrealist Collage in Text and Image, p. 7 
symbolic, collage is a radical deconstruction of the language of the Father, unlike automatism which claimed, at least in its early stages, to privilege the semiotique or presocial language ${ }^{29} . "($ fig. 17)
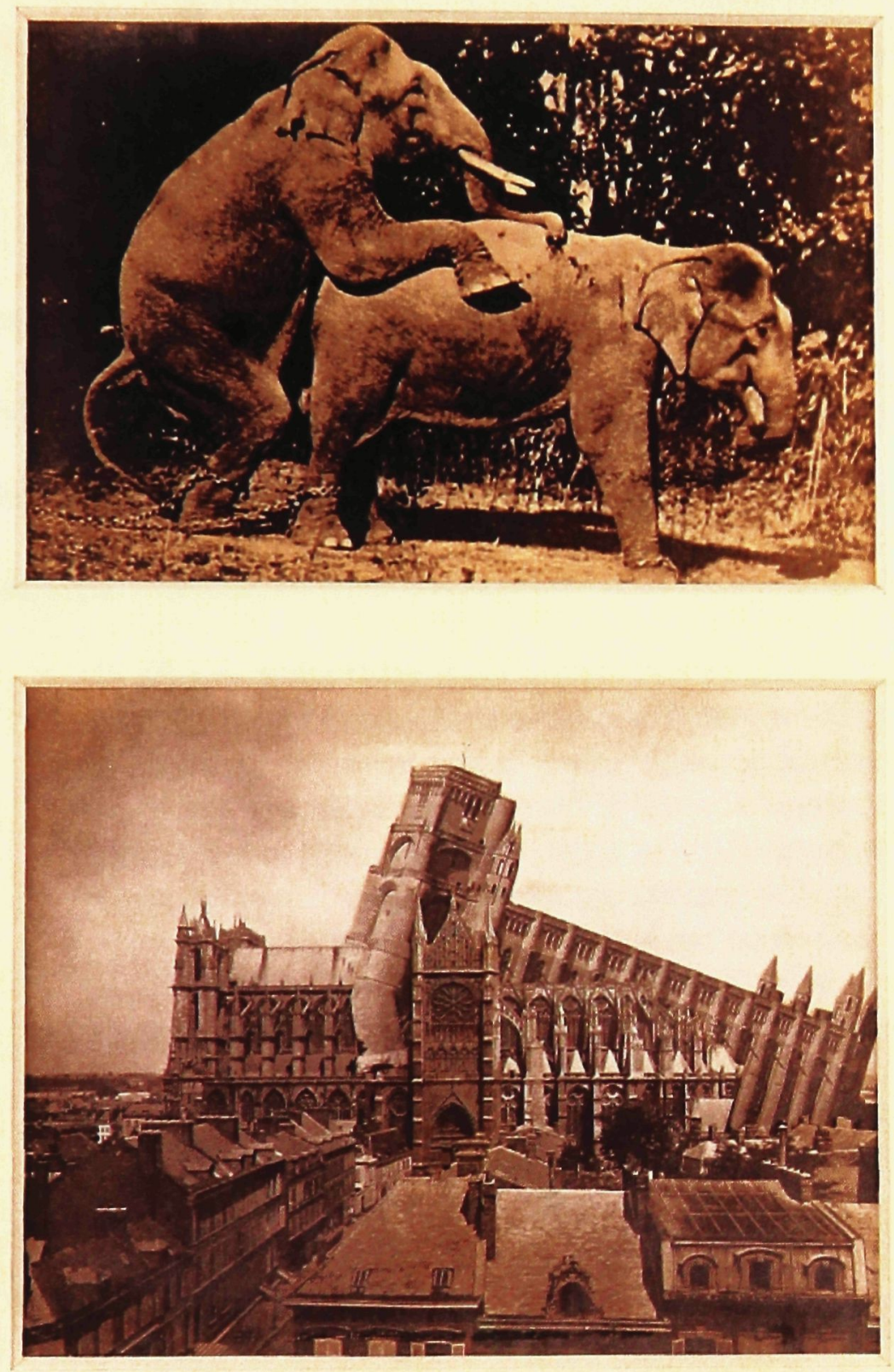

${ }^{29}$ Elza Adamowicz, Surrealist Collage in Text and Image, p. 11 


\section{EMPLOYING COLLAGE:}

The countless variety of found objects chosen for these architectural models was selected for their manipulative qualities that were adequate for the production of these other types of architectural models. It is important to clarify that these qualities are not misinterpreted as literal pieces of architecture. Surfaces, textures, materiality, colour, planes, and volumes are analogous architectural qualities that are considered useful for such models.

Another major concern, in the beginning of the process, was to avoid making arbitrary decisions with respect to starting the model. However, after completing the second model, it became clear that there was no 'right way' to begin these models. In 'Re: Working

Eisenman', Eisenman in this statement supports this condition:

In all processes there must necessarily be some beginning point; but the value in an arbitrary or intentionally fictive architecture is found in the intrinsic nature of its action - rather than in the direction of its course. Since any process must necessarily have a beginning and a movement, however, the fictional origin must be considered as having at least a methodological value - a value concerned with generating the internal relations of the process itself. But if the beginning is in fact arbitrary, there can be no direction toward closure or end, because the motivation for change of state (that is, the inherent instability of the beginning) can never lead to a state of no change (that is, an end). Thus in their freedom from the universal values of both historic origin and directional process, motivations can lead to ends different from those of the previous valueladen end. ${ }^{30}$

Sometimes, it is necessary to start by an arbitrary motion; a motion that is not informed by systematic values. However, this seemingly arbitrary motion has intent "which has an inherent order and internal logic". As long as the motion has intent, it has an objective.

${ }^{30}$ Andrew Benjamin, Re: Working Eisenman, p. 28 
"Every state, it can be argued, has a motivation toward its own being - a motion rather than a direction." 31

Another precedent of models used as generative tool for investigating architectural ideas can be found in the work of El Lissitzky. El (Eleazar) Lissitzky was born in Vitebsk in 1890. Between 1909 and 1914, Lissitzky studied architecture in Darmstadt. In 1919, Lissitzky taught at the art school in Vitebsk, where he met Marc Chagall and Kasimir Malevich. At that time, Lissitzky turned to the Suprematist theory of art and the UNOWIS group, and began to work on a series of unconventional models he called 'Proun'. 'Proun', properly pronounced 'prooon', was used as an acronym for 'Project for the Affirmation of the New'. "These small-scale models where built to offer Lissitzky the opportunity to become acquainted with the fundamental methods and systems of architecture and to play with architectural ideas." ${ }^{, 32}$

In a manner similar to the intention of the models produced in this thesis, the Prouns were tools of investigation that allowed Lissitzky to explore architectural limitations in a non-traditional way. The Prouns themselves were also developed by employing techniques of 'collage'. These Prouns, "were composed of several geometrical elements both two and three-dimensional, dispersed in a manner that created unexpected spatial relationships. Their components were arranged with

31 Andrew Benjamin, Re: Working Eisenman, p. 29

32 Albert C. Smith, Architectural Models as Machines, p.103 
little regard to gravity." ${ }^{33}$ These studies of three-dimensional and spatial conditions were not always composed of two-dimensional planes. In some of his studies, he engaged issues of materiality by introducing elements of metal, cardboard, and collaged paper used in relief within his compositions. (fig. 18)
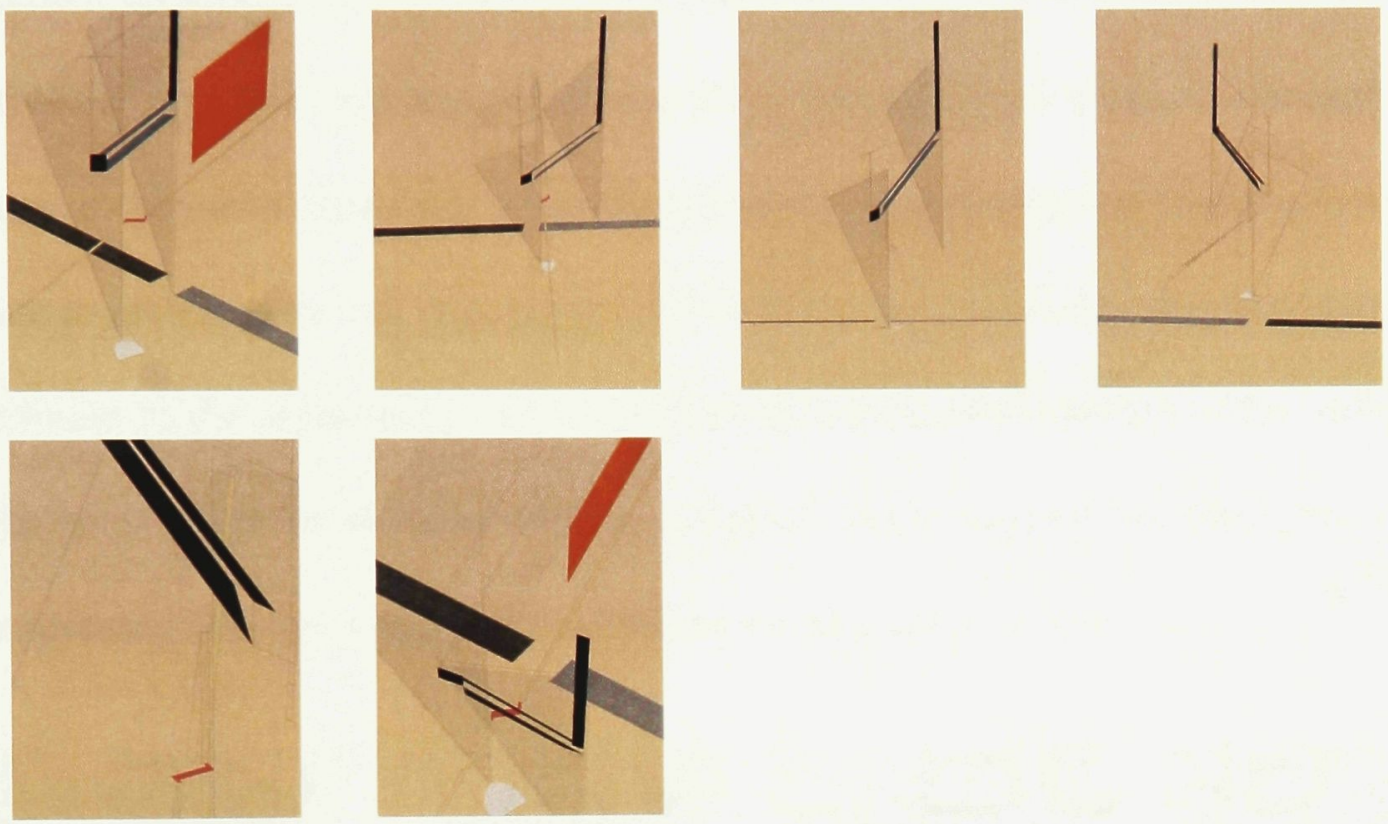

However, this thesis would argue that, like the "analytique" drawings, many of the Prouns were still tied to a two-dimensional surface. As far as visual perception is concerned, they became a frozen moment, a condition frozen in time. These graphic-spatial depictions attempted to perceptually extend beyond their frame, yet they were still limiting as an investigative tool. The "Prouns deployed a space that tended to reach out in front of the picture plane, as opposed to the infinity behind it." ${ }^{34}$

33 Albert C. Smith, Architectural Models as Machines, p. 103

${ }^{34}$ Albert C. Smith, Architectural Models as Machines, p. 103 
An important issue arises when considering the medium utilized while exploring such things as spatial condition and materiality, in architecture. If we truly wish to explore architectural conditions, whether of space, form, or materials, what purpose does it serve to explore these physical moments of architecture on a two-dimensional surface? The argument presented here is that we may challenge these physical issues in a nonabstracted, physical, and immediate system of 'physical sketching'. Investigating by means of 'physical sketching' immediately embodies the physical characteristics that are innate to architecture and engages precise relationships between the maker and the model. The intent of the argument is not to criticize the ideological validity of Lissitzky's Prouns or the basis of the 'analytique' drawings, rather, it is to suggest that their principles might be expressed through a more direct medium; a physical one. (fig. 19)
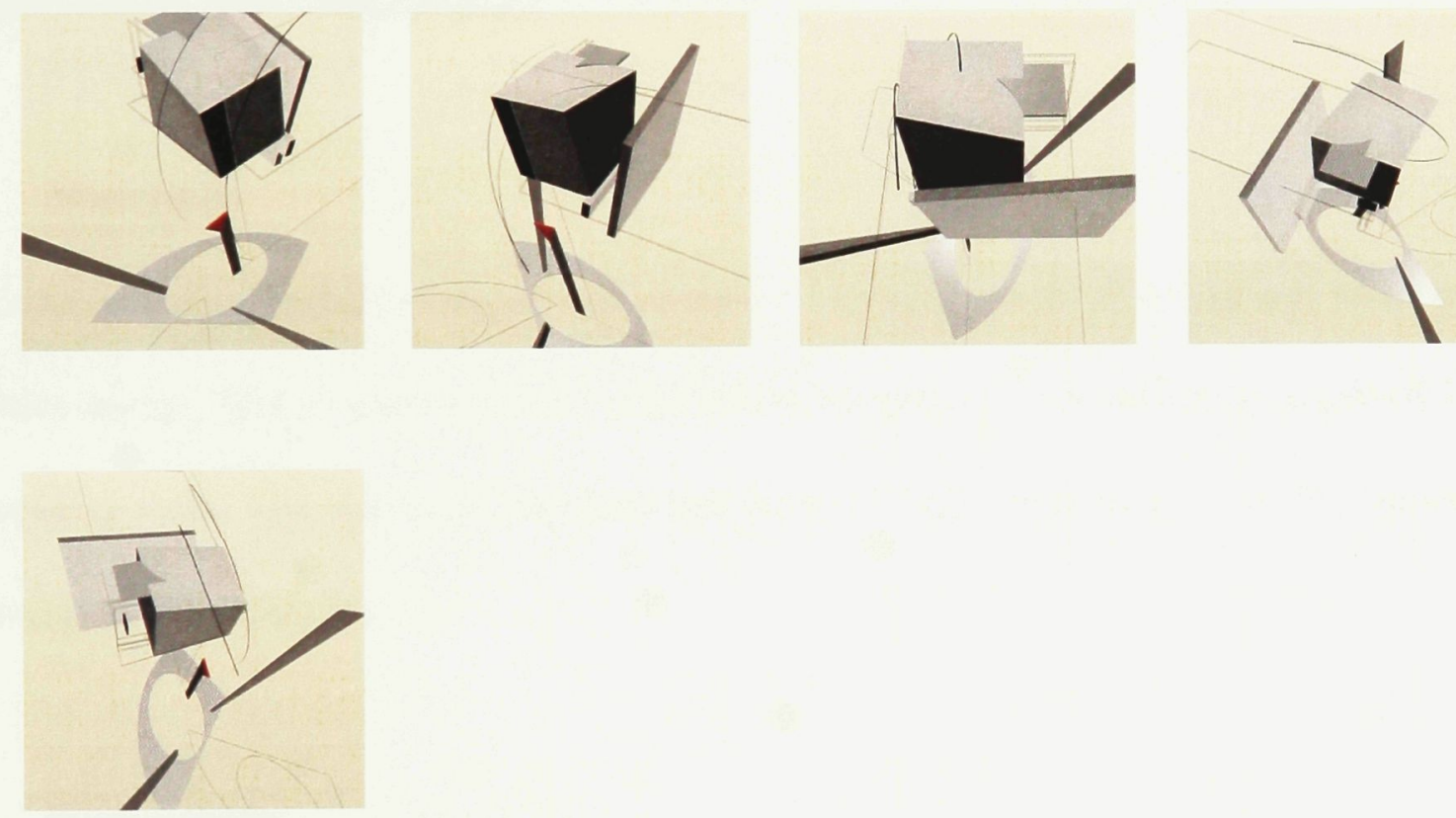


\section{LOGBOOK OF THE MODELING PROCESS}

The next chapter is a thorough analysis of the modeling process. It is an account of all six models examined in a scientific manner. Each of the models is individually studied on the basis of their materiality, forms, spaces, and dimensions before moving on to the next. Doing so has proven beneficial to the evolution of these models, providing a constant reevaluation occurring after the completion of every model.

\section{MODEL "A"}

\section{Comments and Observations}

\section{- Materials:}

Constructed of found/pre-organized objects. Typically made of wood and weathered/aged sheet metal. The connections of the different components are generally made of $1 / 16 "$ welding rods. The fastening methods are done through soddering or simply penetrating the other material. (fig. 20)

\section{- Dimensions:}

The model tries to occupy a similar space that the site occupies at a scale of 1:50.

Maintaining a relationship to scale as a ground rule insures that the model does not exceed a certain size and allows for the other models to be consistent in shape and size. 
Fig. 20

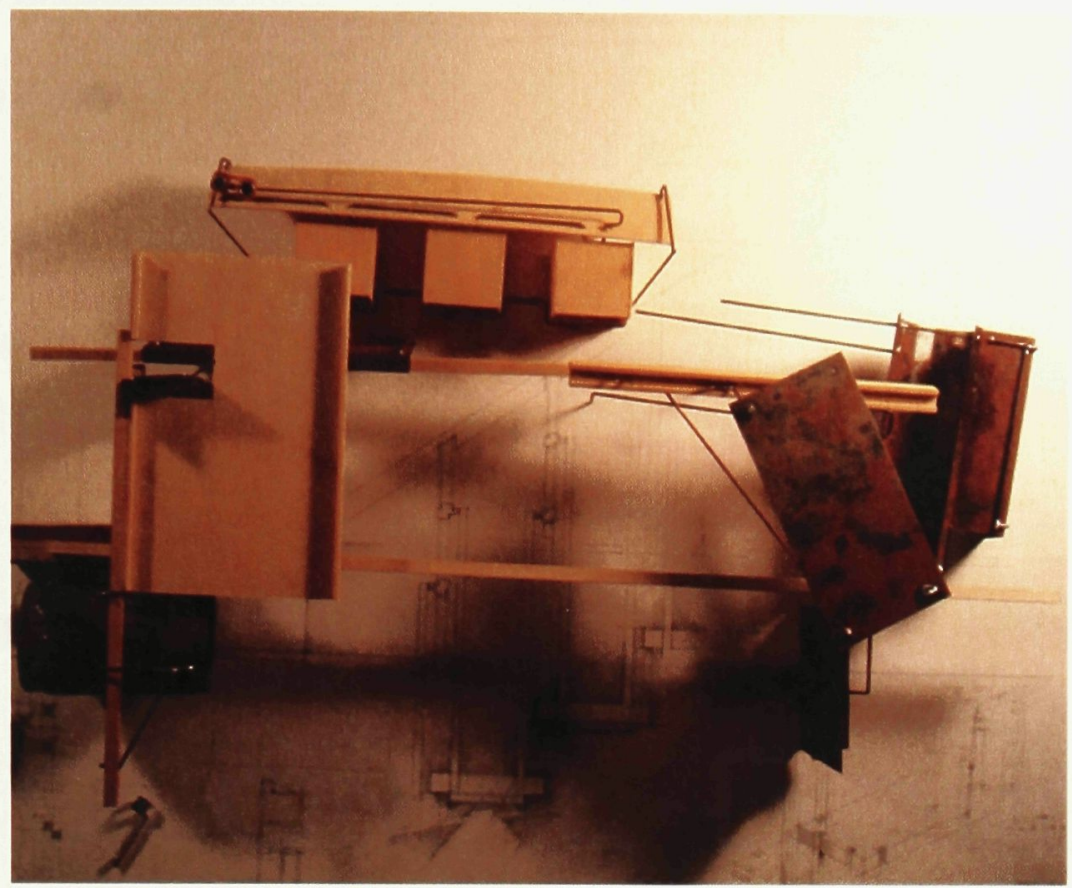

\section{- Form and spaces:}

The objective of this model was to start organizing certain elements (found objects) in order to articulate some spatial conditions as well as organizing certain spatial relationships while keeping the program in mind. The model implies a very subtle hint towards a spatial organization. Certain strong horizontal elements start to suggest spatial division. The confined shape of the model also helps to consider certain particular aspects of the project due to the inherent limitations found on site. The site is thin and neighboured by two existing buildings on each side. 
Model "A" has taken an approach in its assembly similar to collage by using found objects for their potential of interpretation. Although not very substantial as a model, it carried much potential as a means to a process.

\section{- Limitations:}

The lack of materials in this first attempt presents opportunities for joint or junctions to materialize. A diverse pallet of materials would be more appropriate to allow such opportunities to occur. Furthermore, the model was mounted on a board that restricted its legibility to only one point of view. In order for this type of model to be more successful, it should not have a fixed position; rather, the ideal model should be able to rest on all 6 of its sides. Considering this while constructing these models is important to keep in mind for future reiterations. 


\section{MODEL "B"}

\section{Comments and Observations}

\section{- Materials:}

Similar to Model "A", Model "B" is constructed with found/pre-made objects. The material pallet is more elaborated. Its materials range from wood, steel, masonry and cardboard. The connections remain consistent with those of Model "A", and also explore a few other means of connecting: fastening, friction fit, wrapping/holding and hanging. Exploring non-traditional means of assembling traditional materials offers the potential for creative and innovative detailing. That can also be said for found objects/materials and their interpretative abilities. (fig. 21)

\section{- Dimensions:}

The dimensions still respect the site restrictions and remains bound to 1:50 scale in terms of the thickness of the model. It is important that the model maintains a consistent thickness due to its relationship to the site conditions. The thinness of the site/model forces the "maker" to consider the spatial limitations while intuitively designing within them. Understanding the "project" in section is crucial in this instant. 
Fig. 21
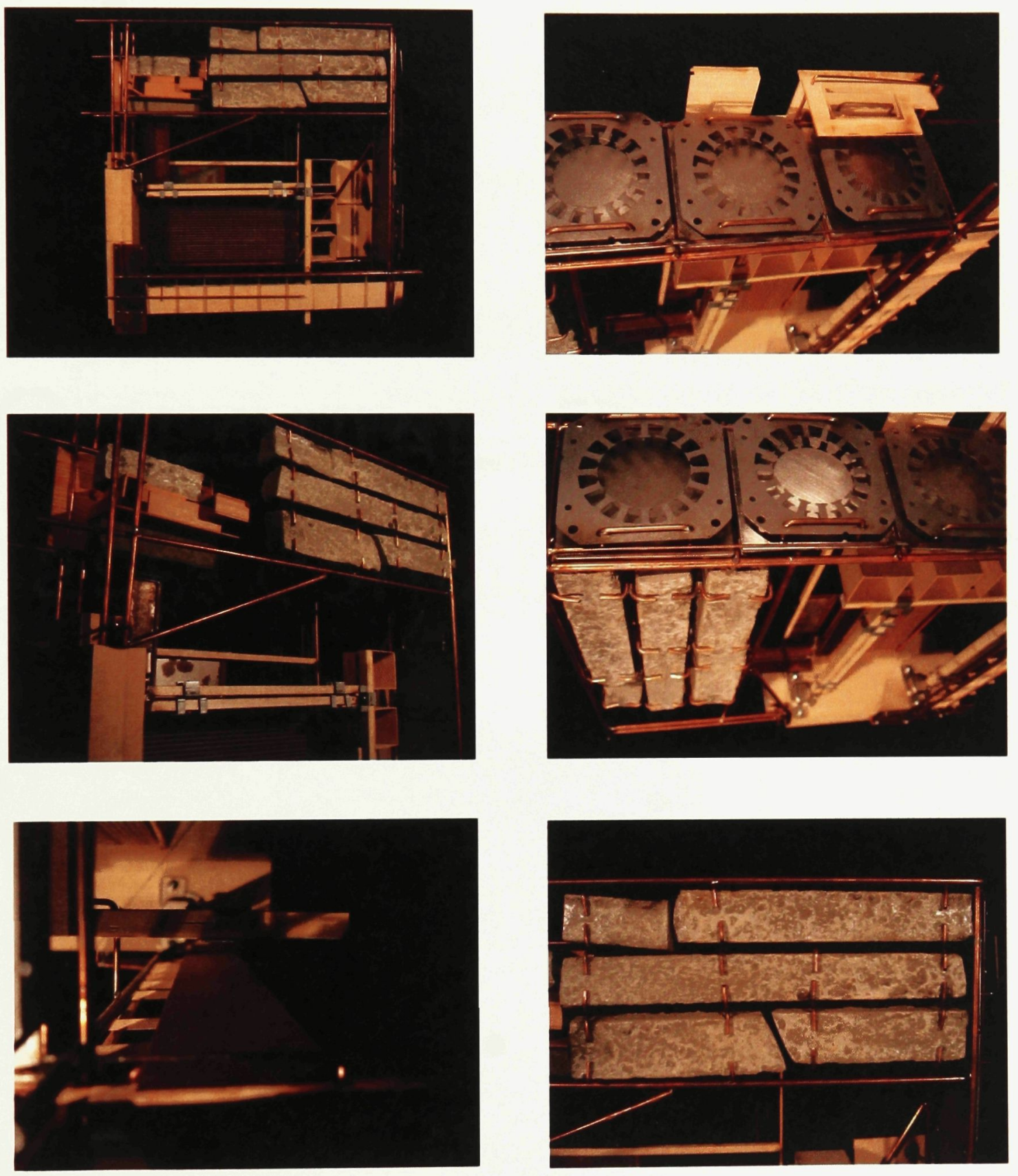


\section{- Form and spaces:}

Model "B" was constructed in a collage approach around a rectangular frame.

Instinctively, most of the "interventions" have occurred on the edges of the infrastructure freeing the space inside. This has occurred because there wasn't any prior framework for the model to take place in. The organization of the surfaces suggests a certain vertical layering as it progresses along the narrow side of the model. A progression through multiple planes and thresholds in a small space may be illusory of a much larger space.

In its organization, the model begins to engage multiple scales, which introduces the viewer to the notion of individual interpretation. It blurs the threshold of our rational mind and allows our imagination to interpret the object and its context. One of the objectives of the Model was to allow more points to sit on all or most of its faces. Model "B" can be sent on 4 of its faces granting multiple interpretations. It is an improvement from the Model "A". (fig. 22)

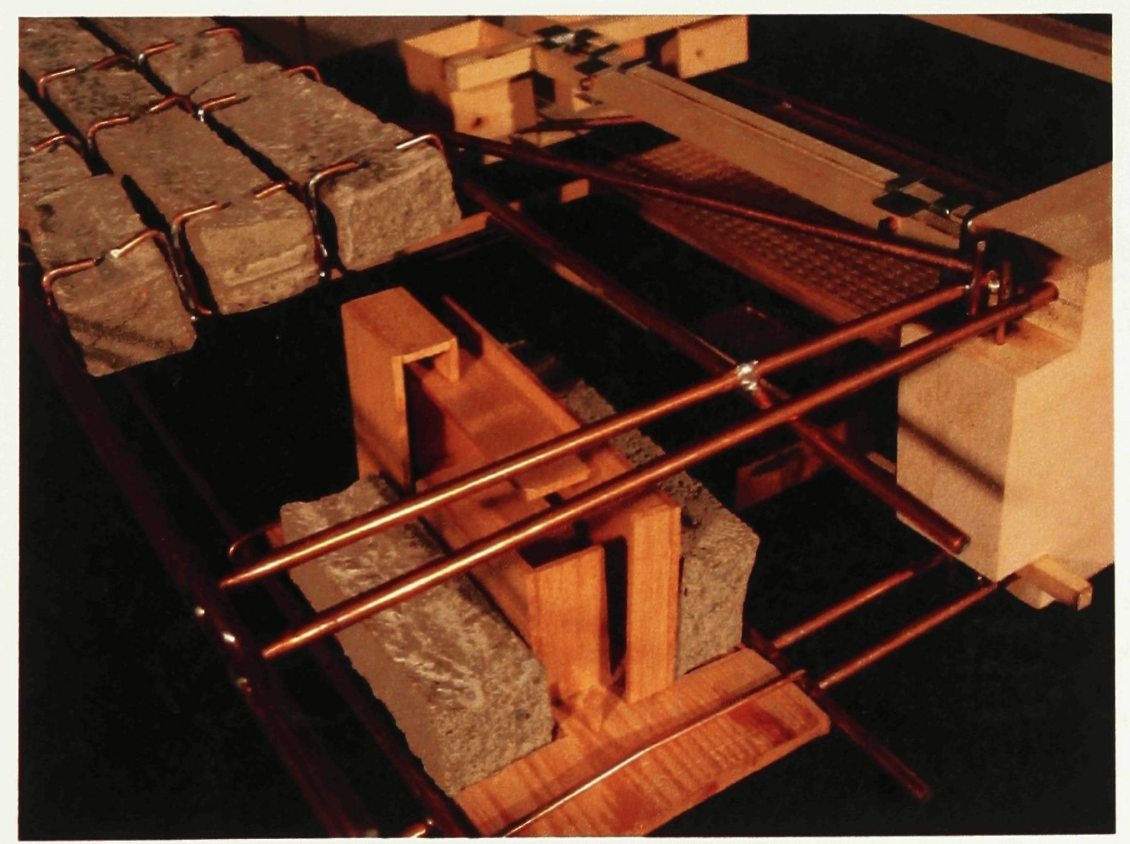


Although one can get multiple readings from this type of didactic model, the piece or object selected for these models contains certain intentions that are nonetheless projected. These intentions are imbedded in the materials as well as the organization of the model. This is where interpretation comes into play.

(fig. 23)

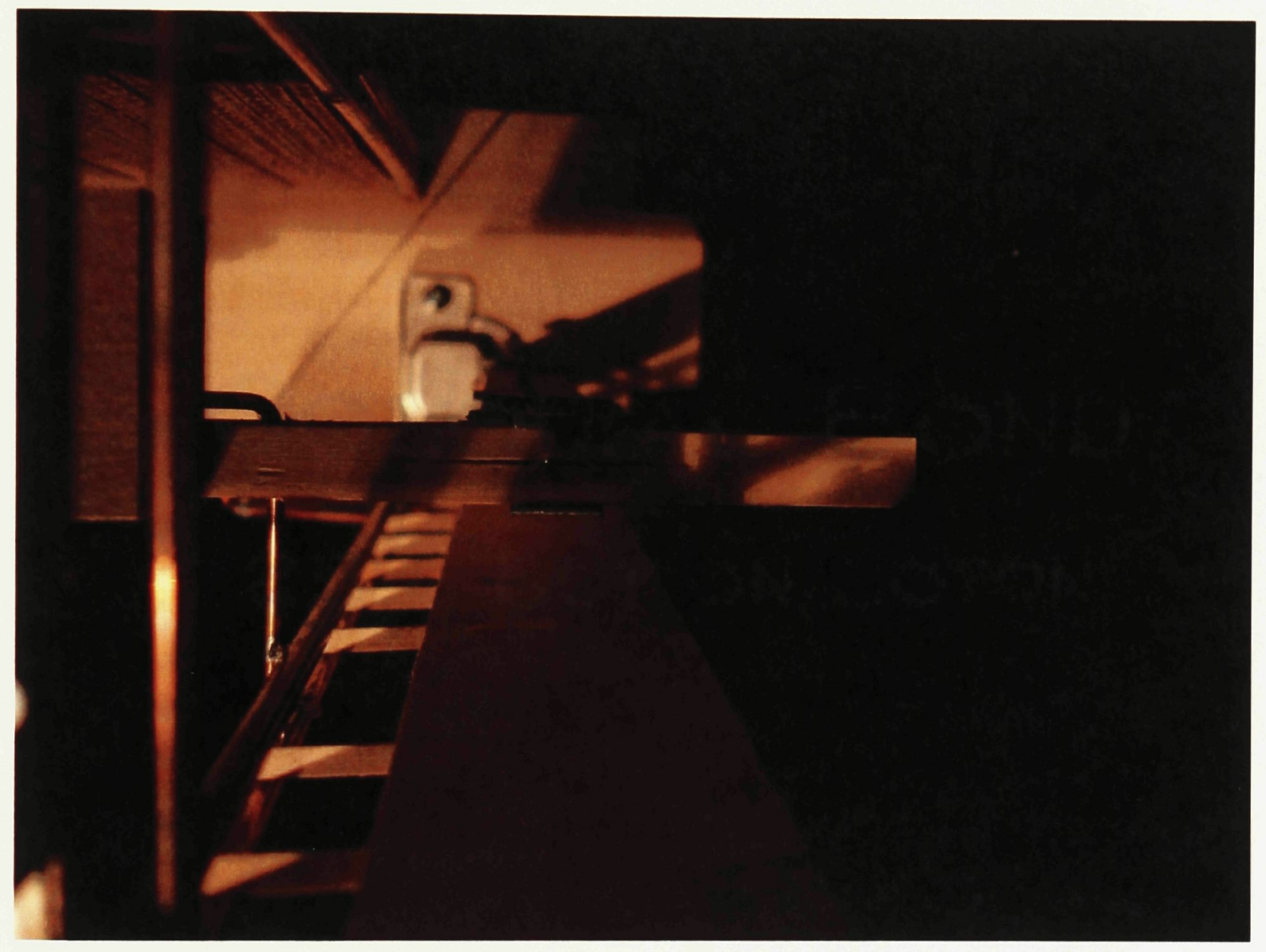

Now that the Model is a little more articulated, certain details or suggestive intentions are starting to surface; such as structural joints, potential masonry detail, porous surfaces, and 
surface treatments. Note: "The quest for origin in architecture is the initial manifestation of the aspiration towards a rational source for design. ${ }^{35}$ (fig. 24)

- Dmensions

$\rightarrow$ the dineusions still respect the site Restrictions sid Rarains band to 1:50 scalle in teem of the thickness of the Moasl.

- "Mochel is borrowed from Middle FeEnut modele, from Italimos modelle, a maded or Mold Rram the Uligar Lstin Madeblus. Madeblus is a diminutive of the Lafin modulus, a diminutive of modus, which meous to measure." pg 62 (Architecturd Model As Maching).

- "A nodel un also offer a tentitive description of ithedey or sustere thid accounts for All itskown peoperties. "Ps reand"

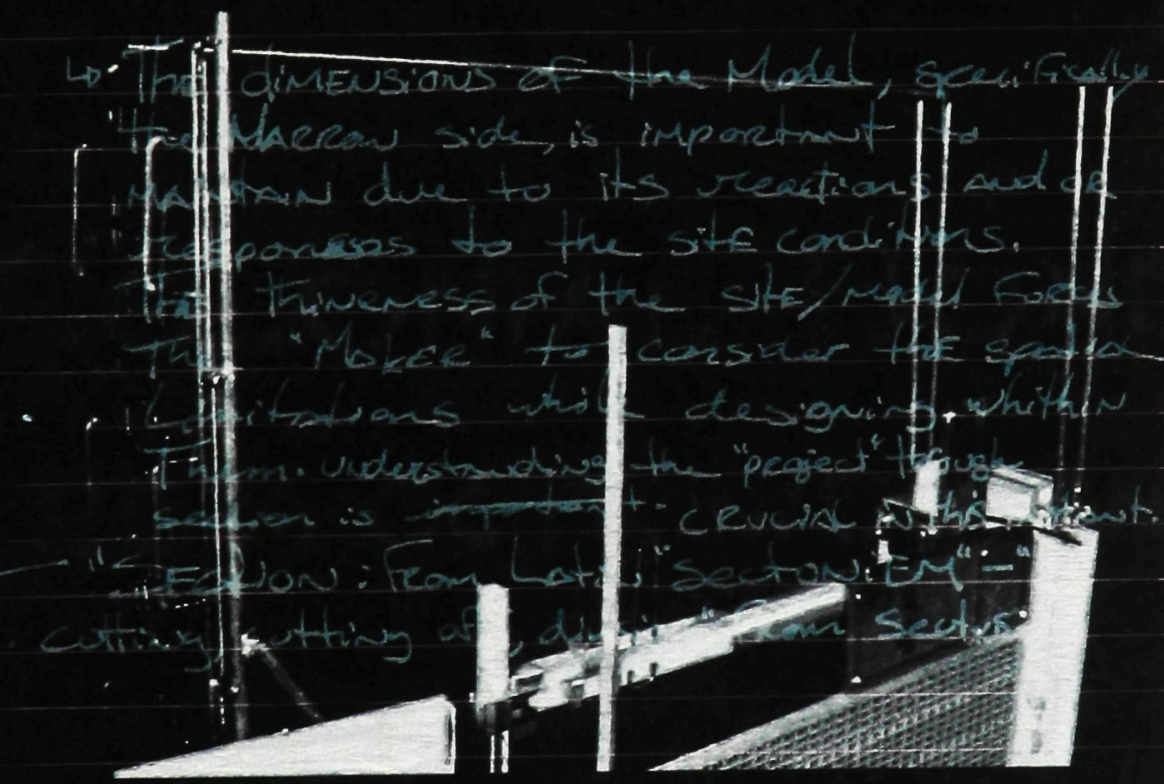

${ }^{35}$ Andrew Benjamin, Re: Working Eisenman, p. 25 


\section{- Limitations and Criticism:}

The fact that all the interventions happen on the periphery of the frame may be limiting the potential for relationships occurring at in the center of the composition; Condensing the interventions could force a more successful and promising space. There would have been richer condition between the components. Constructing beyond the edges has hindered its legibility on all 6 sides. Or what if it wasn't? Questioning the parameters of these models is important and affects the development of the process. Creating these rules to which these models adhere, allows for a certain amount of control over the growth of the models. (fig. 25)

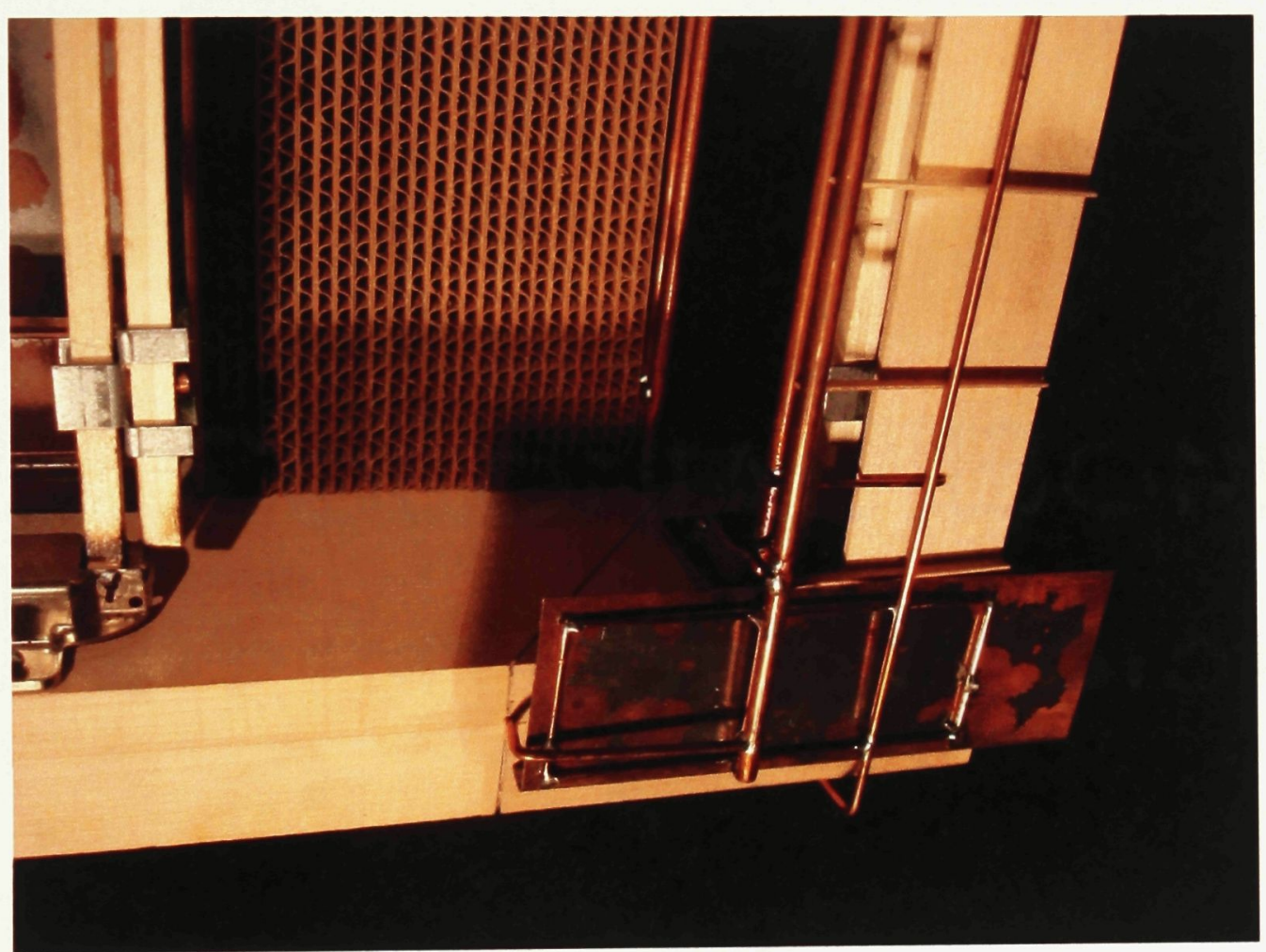


MODEL "C"

\section{Comments and Observations}

\section{- Materials:}

The Model maintains a similar material pallet consistent with the previous models. Copper has been introduced as a new material and was selected due to its rich physical characteristics. Copper is a material that weathers well through time. Its malleability is advantageous when considering craft. It also emits a warm and rich quality of light.

Metal mesh is also a material that has been employed in this model. It introduces ideas for semi-translucent surfaces or screens. This raises the idea of porosity within the context of the project.

\section{- Dimensions:}

This particular Model, along with the next three models, will be restricted to a consistent frame of $4 \frac{1 / 4}{4} 13^{3 / 4} \times 10^{1 / 4}$. The frame is to be consistently made of $1 / 4$ " solid steel bar. While comparing this model to Model "B", it is clear that using a pre-made frame allows the possibility to build more freely within the space of that frame. I believe that building a model like Model "B" forces you to build the "frame" as you are progressing though the modeling. In fact, you unconsciously incorporate the frame while you model. This may explains the tendencies to model towards the perimeter of the frame rather than inhabiting 
the center. This new model uses the space within the frame as the canvas which opposes the building principle of Model 'B'. (fig. 26)
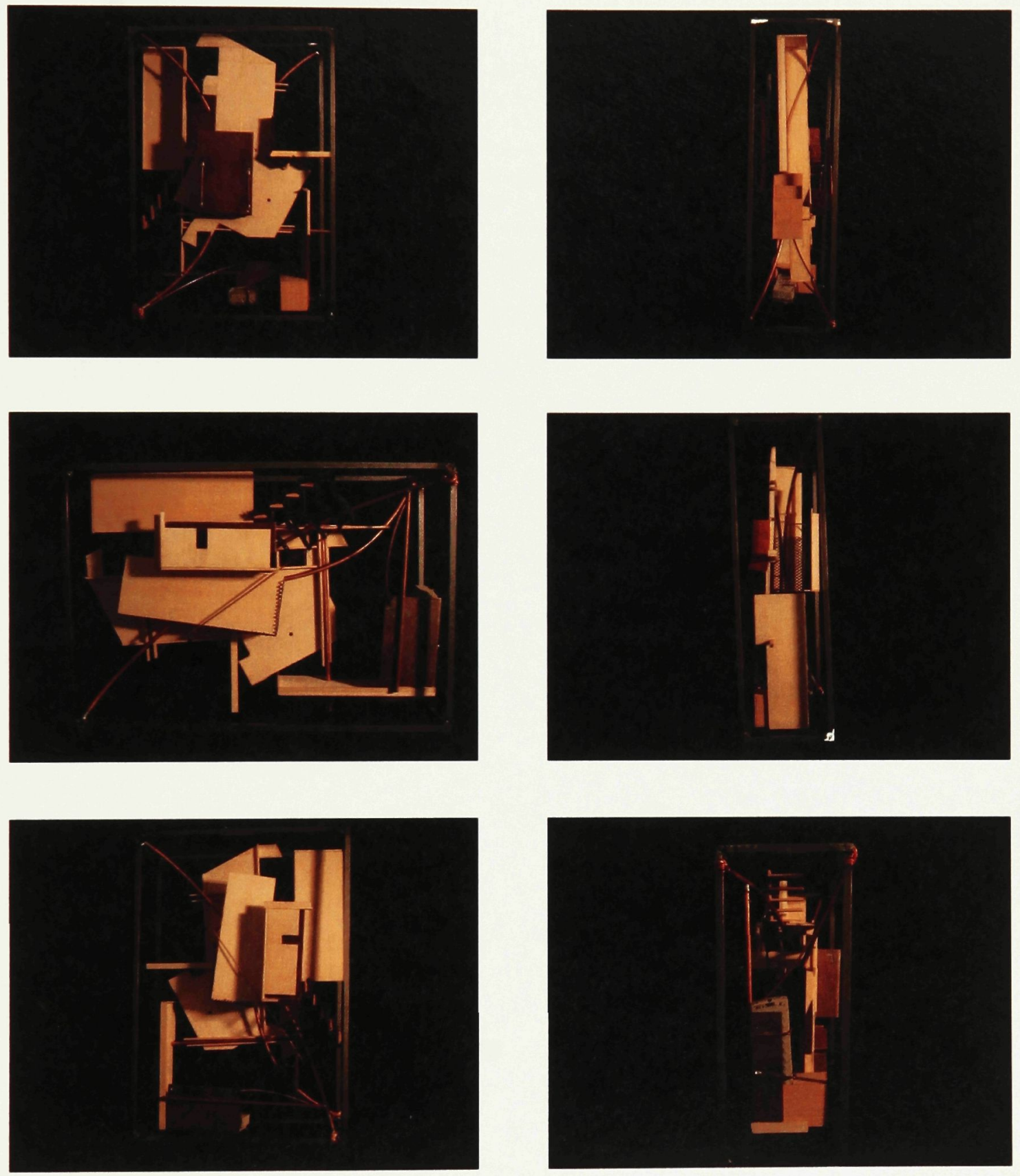


\section{- Form and Space:}

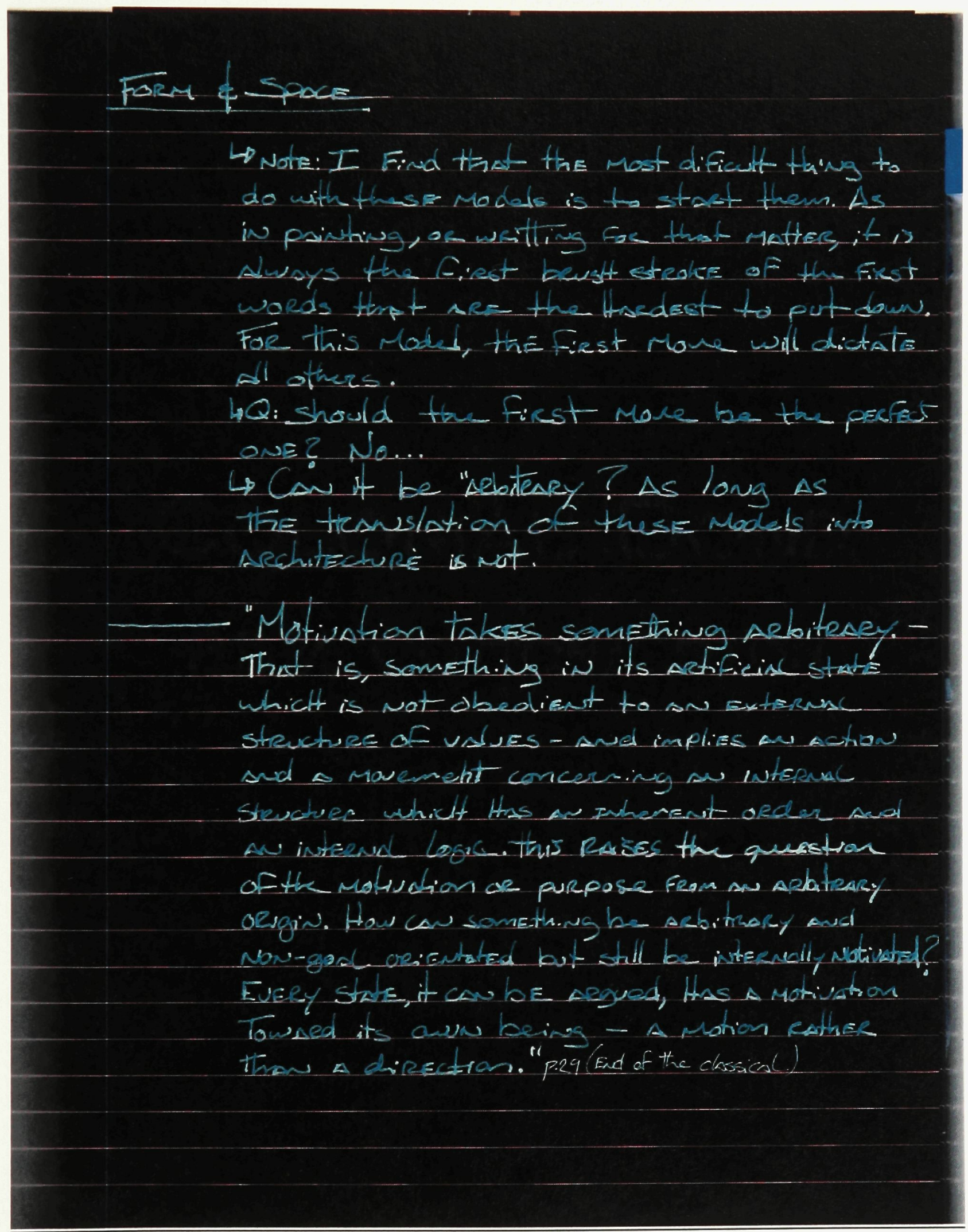


The two first elements in this model were the two curved elements that cut through the space of the frame. Working in the space produced a much richer variety of moments in both material and spatial form. Now that the periphery of the frame is free it is possible to turn the model on all its faces. It allows the viewer to truly engage the model. By using the two curved elements, I wanted to somewhat escape the rectilinear aspect of the model. I don't believe it was all that successful. It somehow has an inherent willingness to be rectilinear. Maybe it's just the maker, or potentially the objects? The initial curved components were selected to develop a more fluid organization. I assumed that starting the model this way would allow for a more interesting distribution and arrangement of the object. Oddly though, not much stemmed from these components. They seem to be mediating the central space, somehow maintaining its uncompromised spatial moment.

In this model, layering seems to be the factor that is generating some of the more interesting spaces. The density of the modeling at the center of the space is also contributing to the existing spatial relationships occurring. It is also offering interesting opportunities for great detail. The model along with this new development strategy is proving to be a great source of architectural ideas. (fig. 28) 
Fig.28

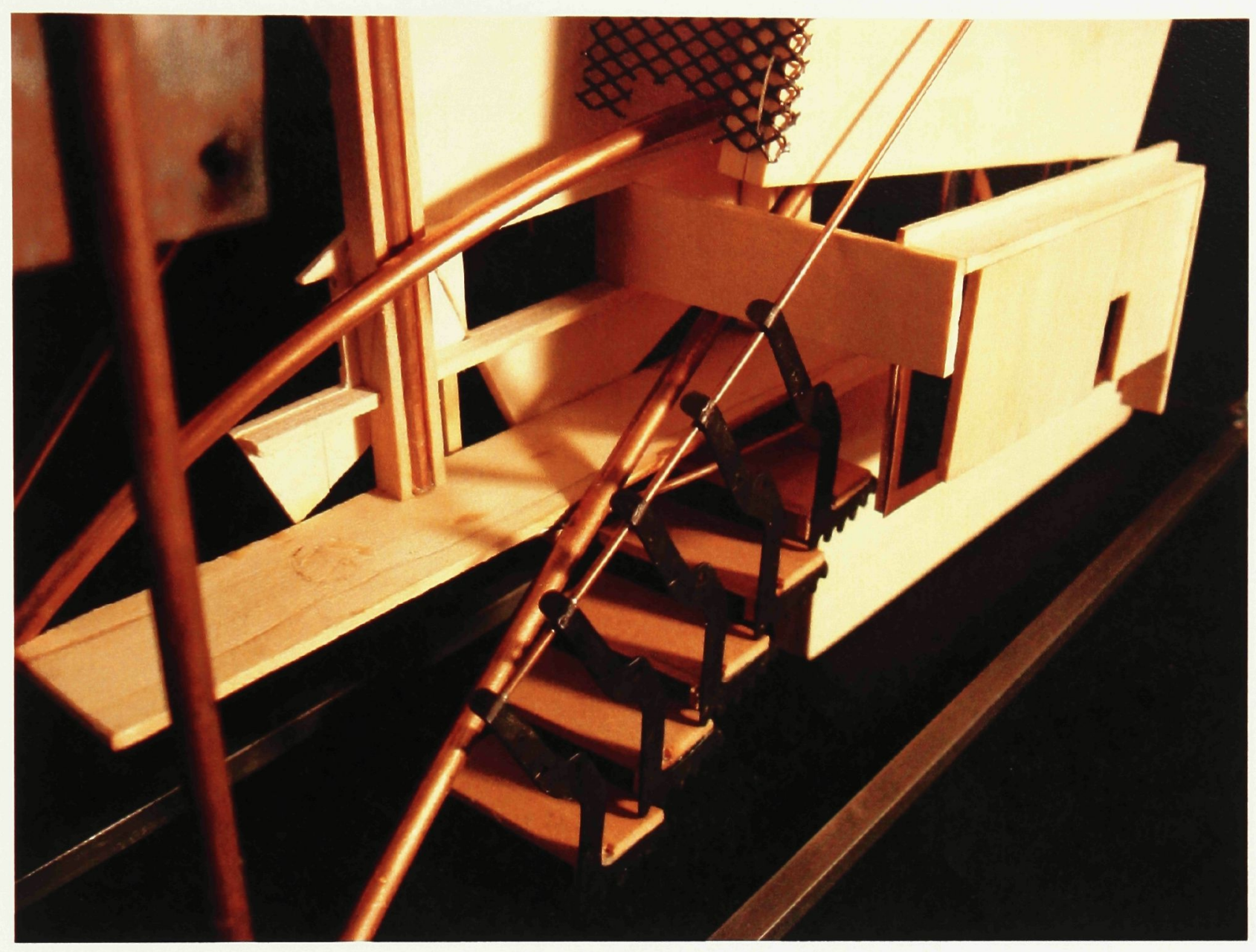

When introducing something identifiable, it starts to put everything around it in context to itself. Ex.: the stair section in this model - it can start to apply a certain sense of scale to other objects surrounding it, which might not have direct relevance to the context of the stairs. This means that these types of identifiable architectural conditions affect tour interpretation of non-related objects that surround it. 
Fig. 29

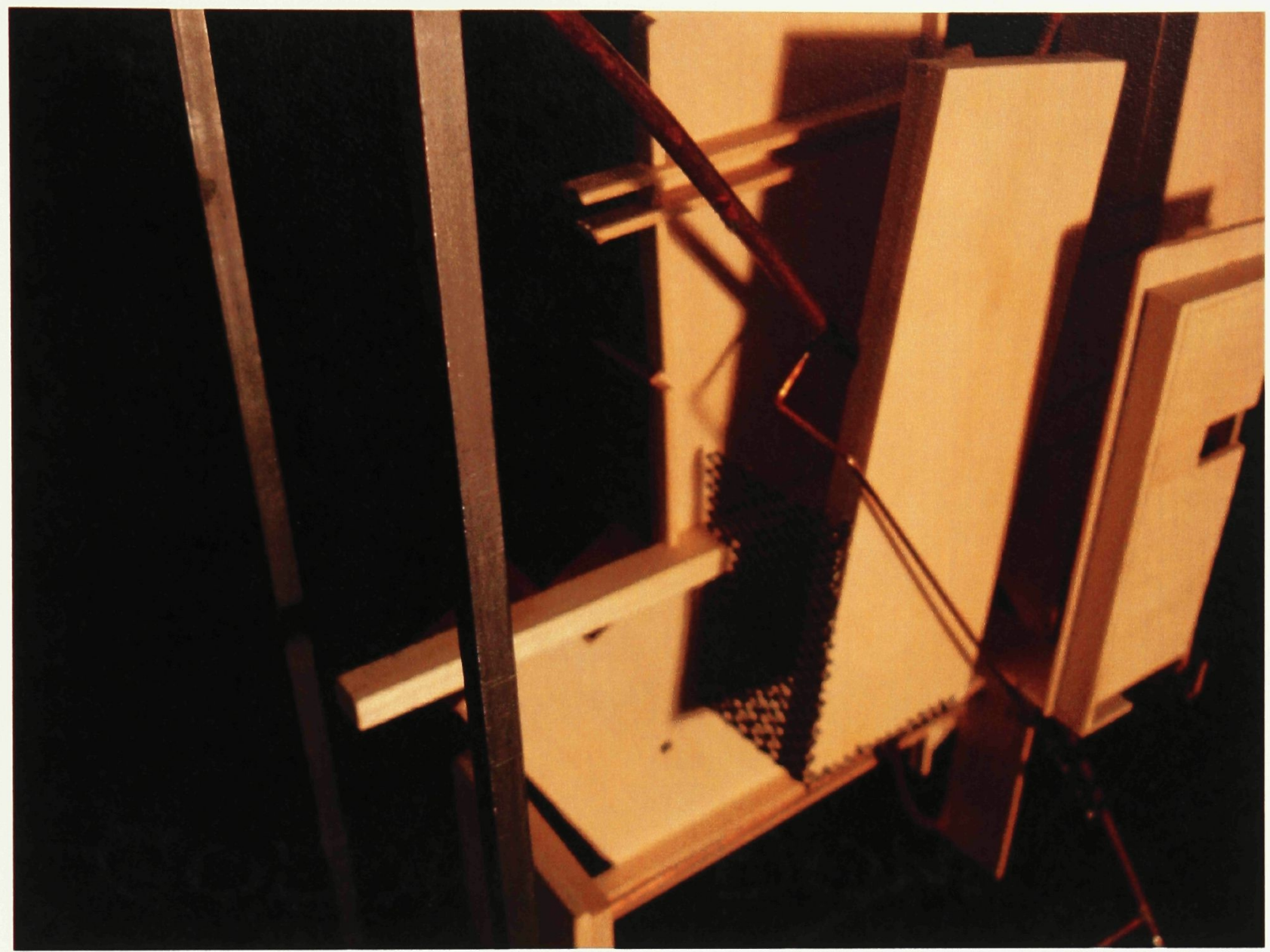

Also, when working with found/made objects, there is so much opportunity in the assemblage of such objects because of their interpretability. The black component that composes the stairs could have easily been interpreted as something other than what I decided to make of them. However, one should not stop here; it should not be literally or formally interpreted as such when translated into architecture. The idea should then evolve through drawing. This process should be applied to all ideas derived from such models. They serve as an instigative tool for rich architectural ideas. The articulation of these ideas is up to the architect/interpreter. 
Fig. 30

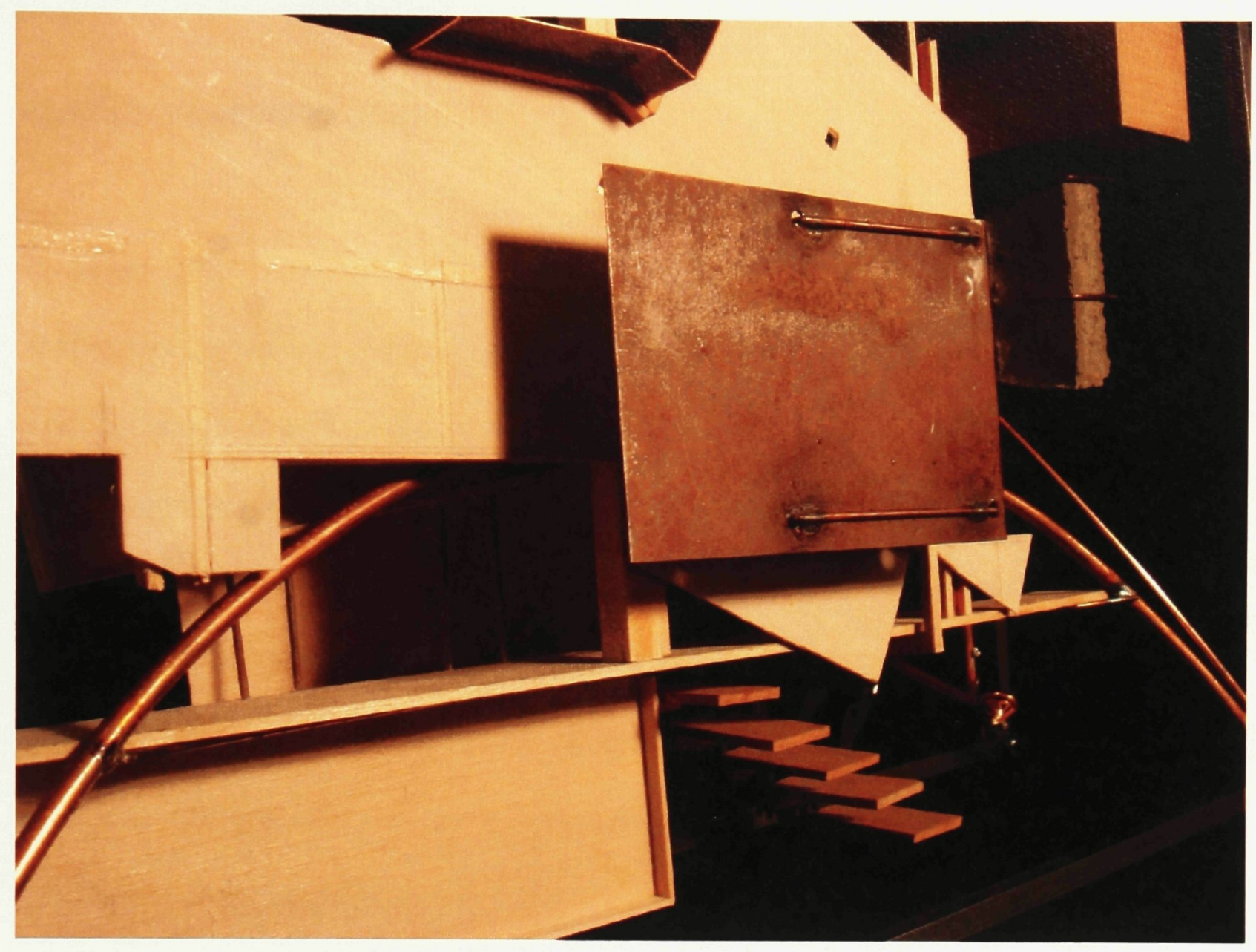

\section{- Limitations and Criticism:}

At what point can these models become too dense? It would be interesting to see if one can saturate a model with to many objects. Materiality could be pushed a little more in the next model. If these models seek to brake from their linear order, I think that one needs to stray from using objects that are rectilinear! The least successful part of this model is the organization and the relationship of the concrete piece. It seems to be too disconnected from the other parts; it is weak because it is lacking dialogue with the rest of the composition. 
MODEL "D"

\section{Comments and Observations}

\section{- Materials:}

This model carries over the same materiality as the previous models. It starts to introduce a thicker gauge of steel. Glass tile was added as a new material. The idea was to use backed ceramic tiles with solid colors and a lustrous or glazed finish. The intent was to use ceramic tiles due to its relationship to the making process. I was interested by the fact that they are 'backed' and that they undergo a similar process than pastries do. This also led me to question other materials that are produced under similar conditions. Ex.: bricks, terracotta. The glass tiles are the best example that I could find that had some of the initial qualities I was looking for.

The objects chosen for this model seemed to be a little bit more articulated, architecturally. The composition does not appear to be as successful as the others. There is also a greater density within the model. The pieces chosen for this particular model might be too defined.

\section{- Dimensions:}

Like the previous model (Model "C"), it is restricted to the same parameters of the steel frame. By maintaining a consistent framework in which to build these models in, it is becoming apparent that this systematic approach allows for a more consistent study and 
analysis of this particular process. This method allows the models to evolve in a parametric environment and ensures that they can secure as a basis from which one can draw relationships. (fig. 31)
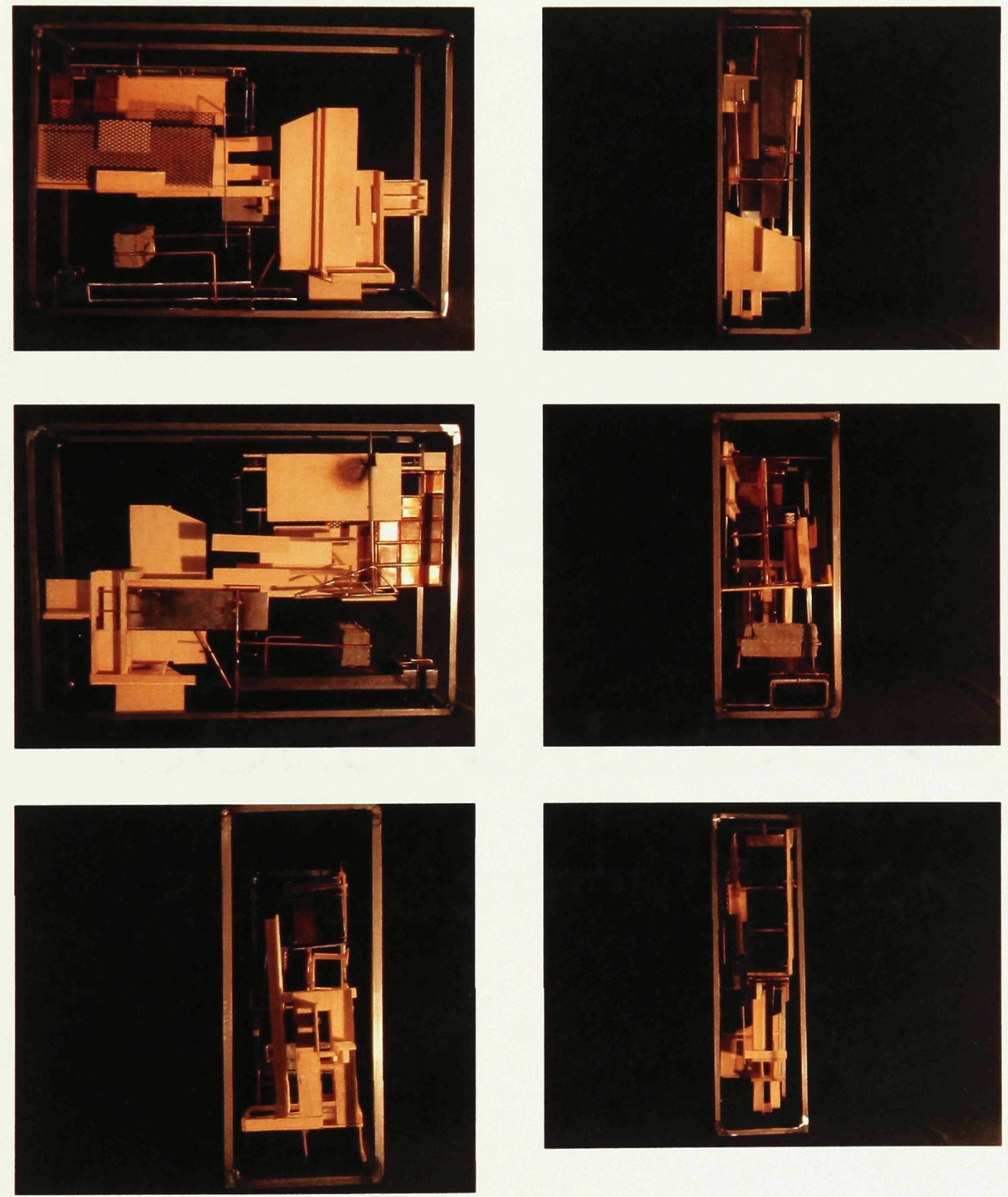


\section{- Space and Form:}

The model explores multiple types of surfaces using materials such as copper, concrete, black glass tile, steel and metal expanded lath. The density of the model is more intense and potentially richer architecturally. However, there is so much happening within the space that I question if density in this case, is a positive or negative aspect. Perhaps this model and the objects selected are too architectural. Is this a negative aspect? This brings up an interesting point on the limitations of certain objects used in this type of model. Can there be such a thing as an over-defined object? The moments that I think are more successful are those that are not so defined. (fig. 32)

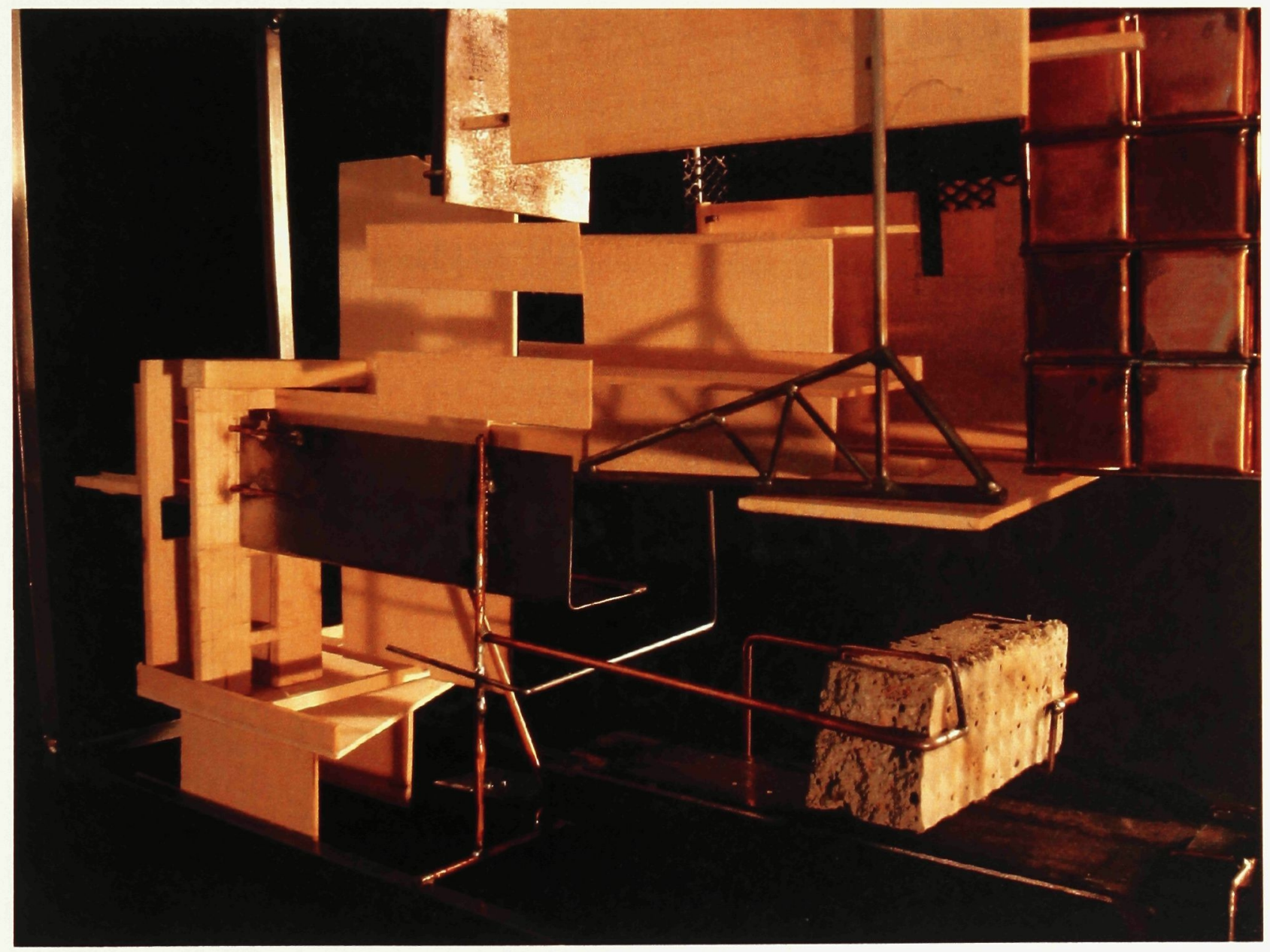


The model has some moments of value. Once photographed and layered with drawing, it may provide a good source of ideas in terms of understanding the spatial relationships of the space through section. The floating concrete element is interesting in its potential to become many things. One can start interpreting it as some sort of seating element or perhaps a table. The copper component provides great insight to the rich qualities copper can start to offer. Because copper has degrees of weathering, creating a surface of varying textures is possible. Although the glass tile was not what I intended to use, it still possesses great characteristics and could be an interesting surface if used carefully. Also, the metal lath is a promising material. This material only physically separates a space but allows everything else through; sight, sound, and smell. Could this type of material be used to emphasize certain qualities in relationship to the program? To hear the sounds of the pastry chefs working and to smell the freshly-cooked pastries throughout the Architecture. At moments, catching a glance of a chef, at his dressing station, putting the final touches on the finely crafted cake or pastry. These phenomenological moments and qualities are important to capture in such a program otherwise, it's just another building that doesn't respond to its purpose.

\section{- Limitations and Criticism:}

I think that the pieces are somewhat too defined and limit the interpretability of the model. Furthermore, it is not as fluid as Model "C". Some of the moves seemed a little "forced on'. Some parts are successful, but as a whole it does not appear to be coherent. 
It would be nice to introduce some colours into these models and maybe some other materials - perhaps fabrics? Overall, it is important to be more selective of the pieces we use to avoid making a model where the parts are overwhelmingly defined and provide too many set parameters. It is also important to "read" the context of the composition before adding more to it. There needs to be a certain "flow" to the model. By consulting the previous model, perhaps the larger gestures are the defining elements that help to determine the future direction of the model? (fig. 33)

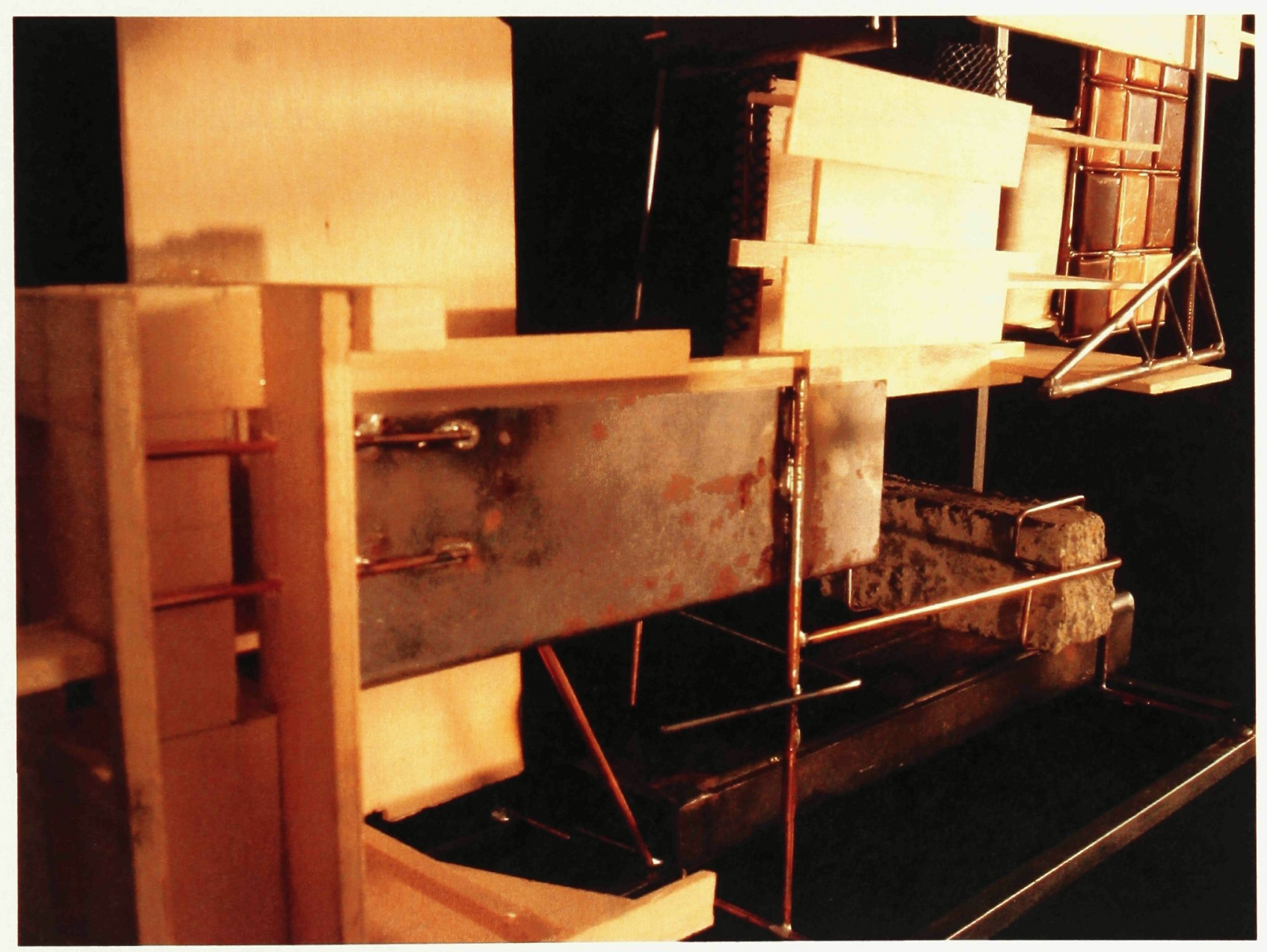




\section{MODEL "E"}

\section{Comments and Observations}

\section{- Materials:}

This model starts to incorporate colour in the composition. It is the same type of tile used in the previous model. It is glass with a highly glossy finish. The initial intention was to use a tile with a gold leaf finish, however I could not find anything like it in Ottawa. One of the main gestures, which was also the first object in the composition, is a piece that is a part of a baking device used for baking crusts for cakes. It is made from galvanized metal. I purposefully kept the latch (see fig. 36) and made it a part of the composition for its rich potential.

Concrete keeps reappearing in these models because I want to maintain a presence of "masonry" without precisely specifying one in particular. It may seem as though I'm selecting concrete, but my intentions are to propose something of a similar nature.

\section{- Dimensions:}

The two previous models have proven successful in the use of consistent framework. I decided to produce the next two models using such frames. The next models will be a part of the same series for reasons of comparison. 
(fig. 34)
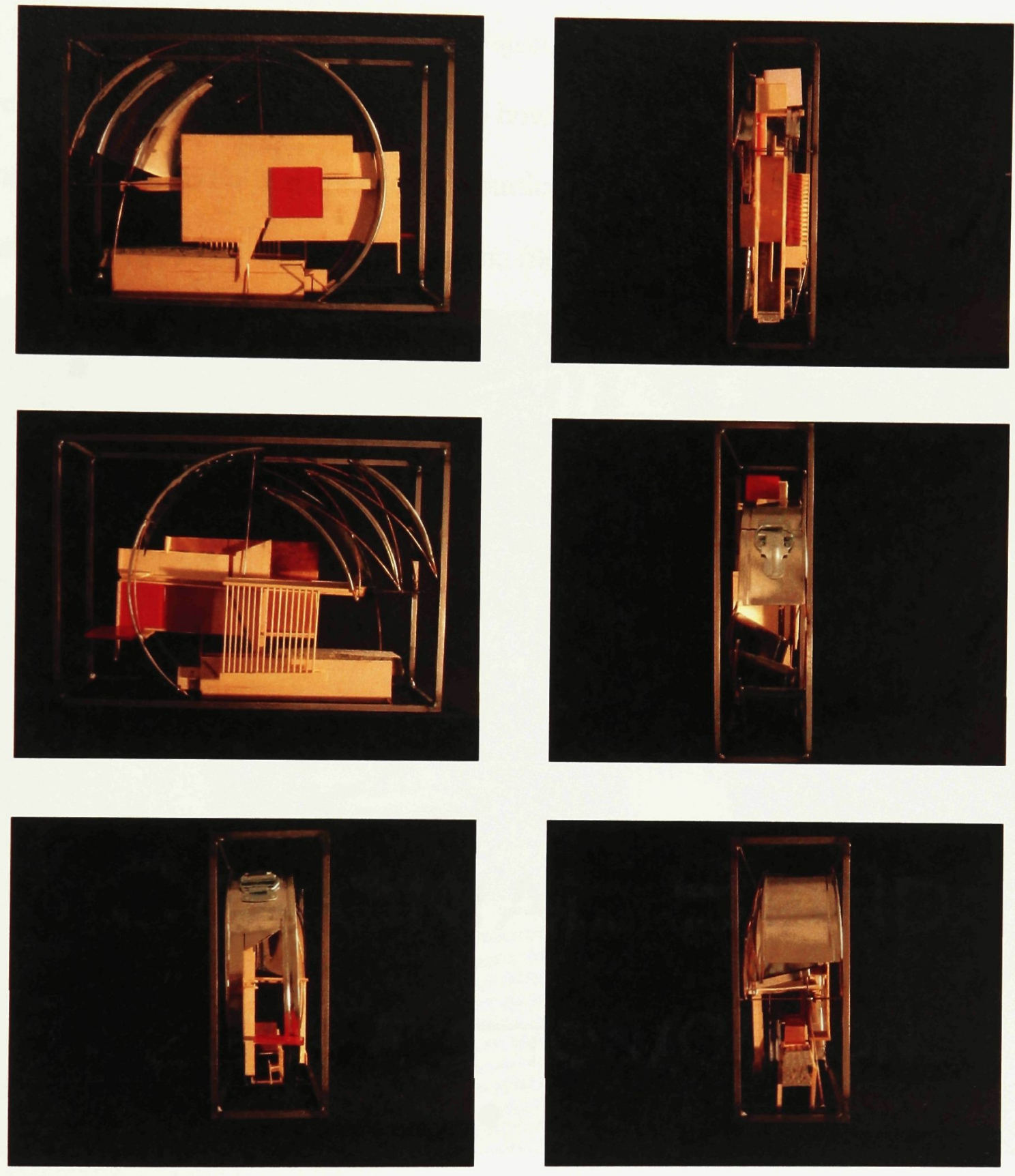


\section{- Form and Space:}

For this model, it seemed appropriate to begin in a similar method in which Model "C" was started. The use of the curved metal object was an interesting way to start because there wasn't any preconceived notion as to how the piece was going to work within the frame. Also, due to the ambiguity of this particular object, it inherently refrained itself from being 'slapped on' to the composition. (fig. 35)

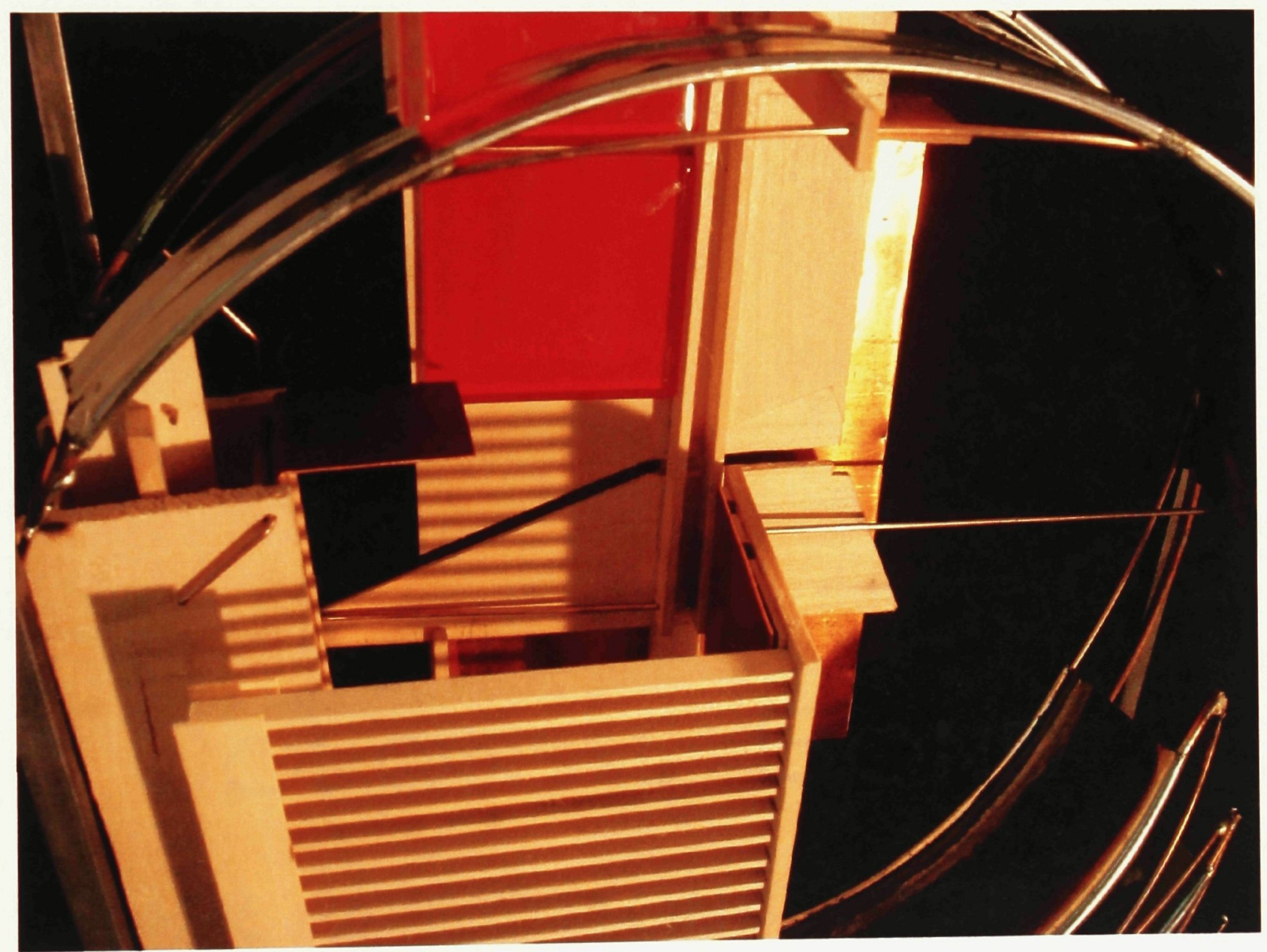

At this point in the process, I believe that there is an emerging strategy that is embedded within the action of collage that may provide a coherent and fluid model. Once this very 
solid gesture was established, I felt that it was necessary to counter balance it with an orthogonal one. I want a good diversity in the composition, but I wanted to avoid creating a mess of curved element. By adding the rectilinear piece, it gave me an opportunity to break away from curved elements. I am also very intrigued by the spatial moment where the large curved component meets the wood plane. It's a very interesting threshold condition between two distinct elements of the model. The coloured tiles seem to help highlight the transition. Maybe that could be translated into a detail. (fig. 36)

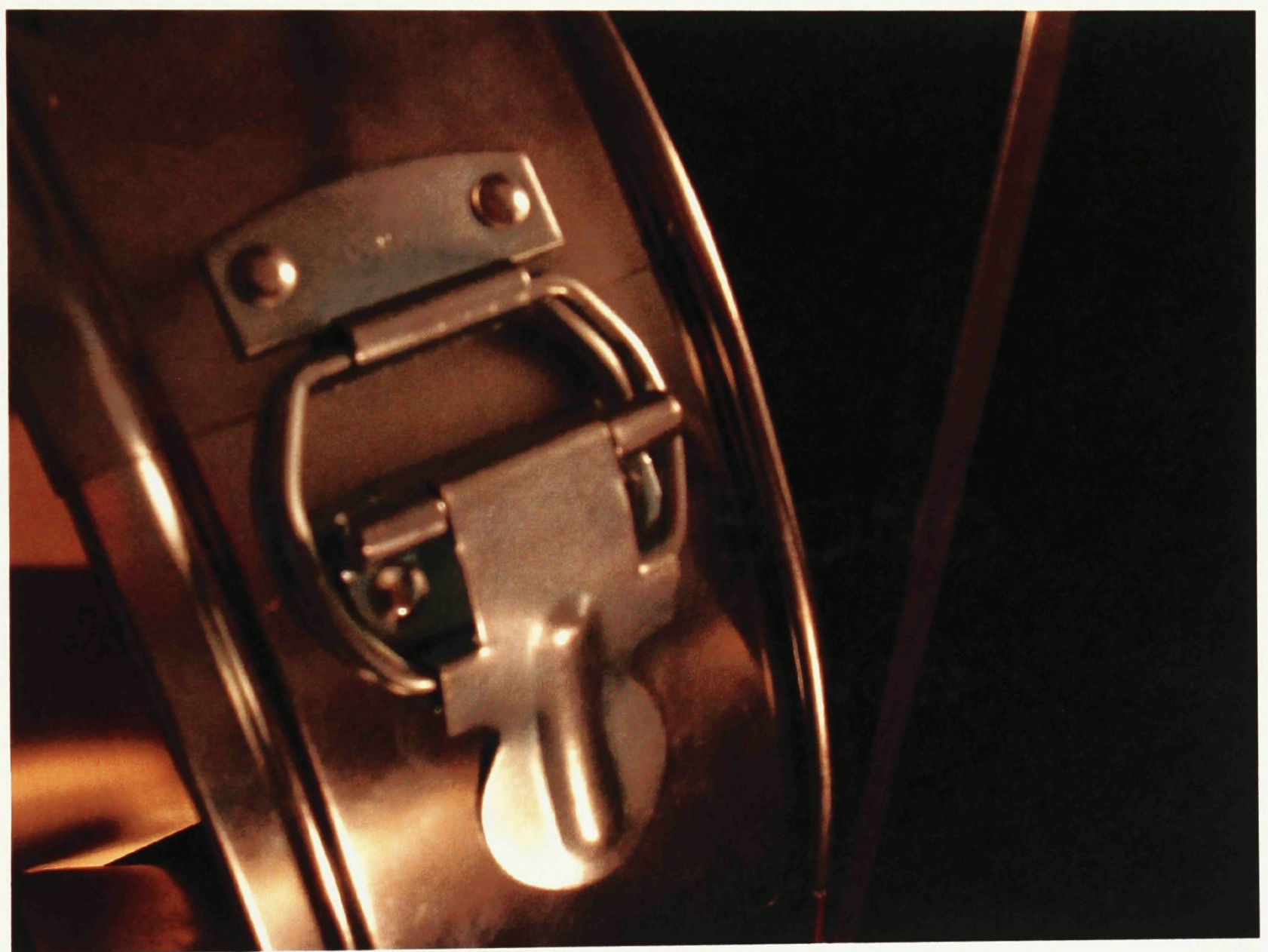

The lattice work is a reinterpretation of the metal lath that was present in the last model.

Furthermore, the lattice offers an interesting screen that selectively allows certain points of 
view. This also depends on the depth of the screen. The reflective qualities of the tile surface offer interesting spatial qualities because it can extend the space through its reflective properties. This may be of use specifically when dealing with a site that has width restrictions such as the one in question. (Expanding space through reflection) The copper also shares some of the same reflective qualities that the glass tile has. Looking at this specific area (refer to image) it starts to suggest several ideas about a potential elevation/section condition facing the street. It questions the potential of the entry sequence and its relationship to its connection to the street. The model suggests the ideas of "slippage" occurring through a layering of planes. Through the use of such "slippage", one can start to introduce rich moments. (fig. 37)

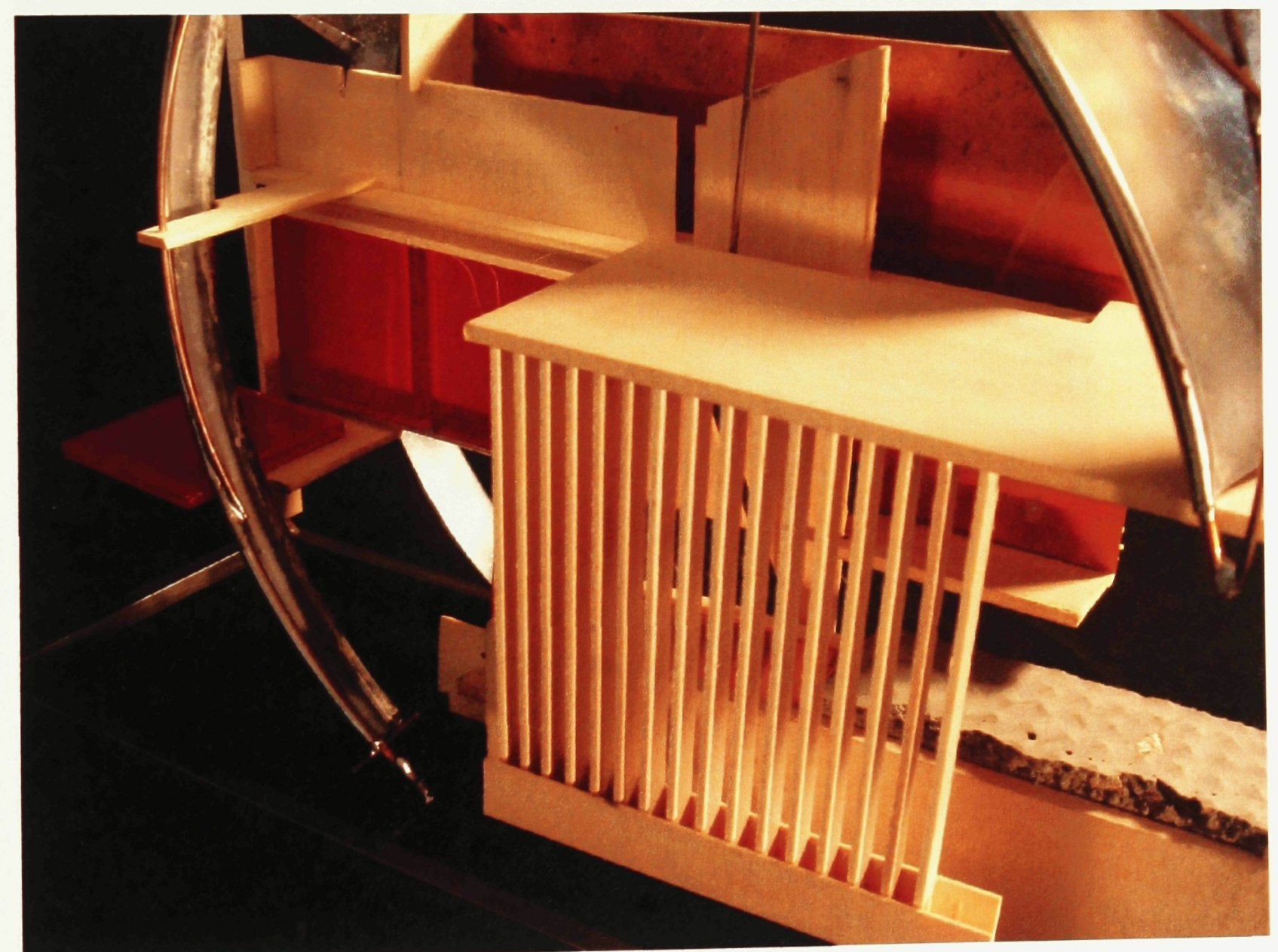


Also, the model shows interest in the junction of two different materials. The model only suggests the possibility of the two materials joining, but the resolution of the metal would be elaborated at a later time. This occurs at several places within the model: between concrete and wood, concrete and copper, glass and wood, wood and copper. (fig. 38)

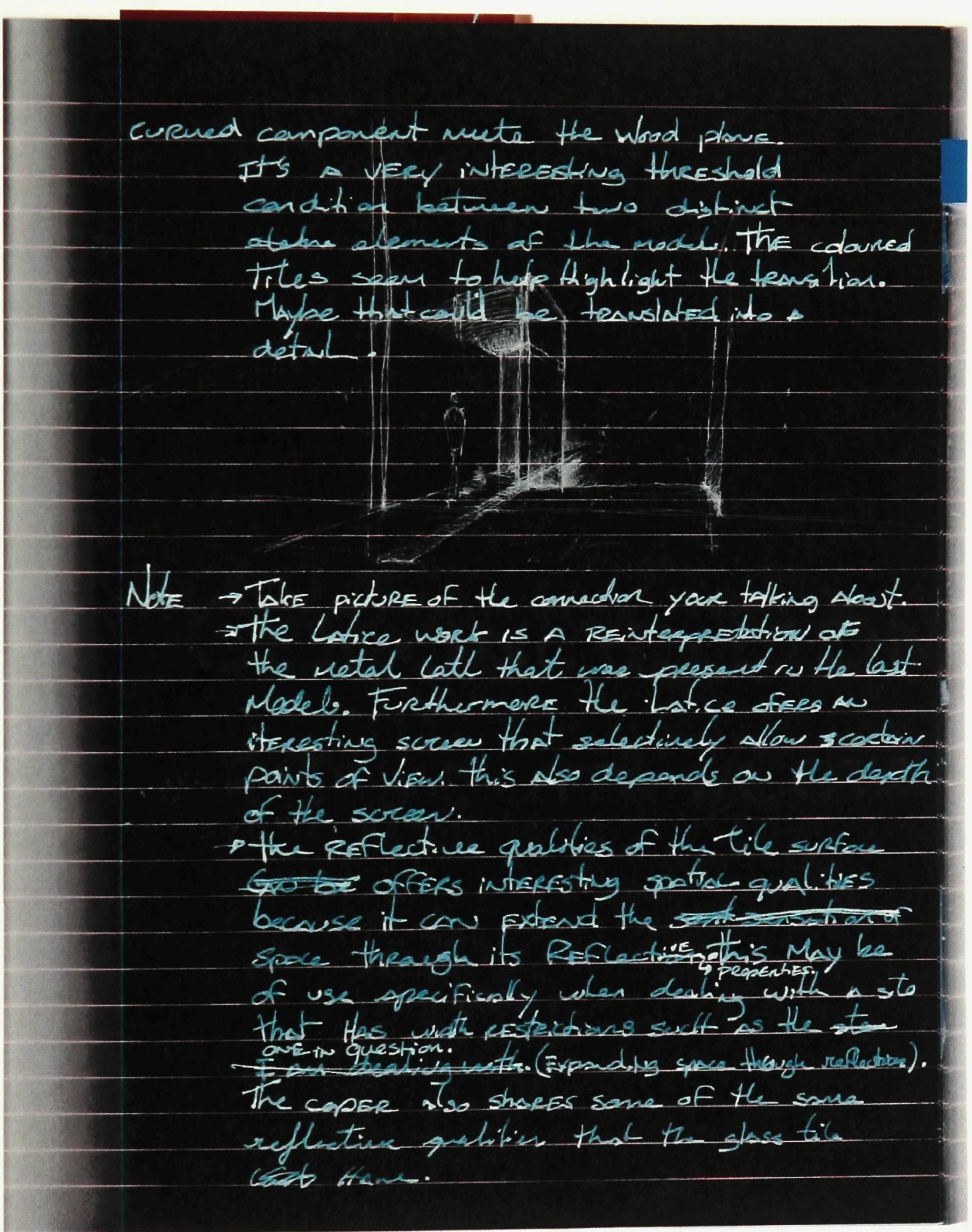




\section{- Limitations and Criticism:}

Apart from not being entirely pleased with the outcome of the selected tile, and maybe the placement of them, I believe the model is somewhat successful. I was really hoping to get real clay tile of specific colours, but reverted to what was available. So I opted for something similar. I could have pushed for more objects in the composition.

Would it be possible to introduce more materials? The recurrence of certain materials seems to suggest that they will maintain a presence throughout the project. If not, could the next model introduce such alternative materials? 
MODEL "F"

\section{Comments and Observations}

\section{- Materials:}

For this model, I have selected a few new materials to use in the composition. I decided to use 2 different types of wood - Bass wood and Cedar. The reason for the cedar is that I was considering the sense of smell in relationship to materials that could play a phenomenological role in the architecture. Also new in this model is perforated sheet metal. It is another material that offers similar characteristics as the expanded metal lath. It is a material that can easily be layered to control sight/light conditions. In the previous model (Model "E", I expressed interest in looking into other material such as fabric.

Similar to fabric, steel chain mail seemed to be fitting because of its similar characteristics to cloth or fabric. The structure of it is similar yet much stronger and could be used as an elegant surface in a space.

\section{- Dimensions:}

The model still respects the ground rules set by Model "C". Every gesture remains within the boundaries of the framework. If we could consider scale as something that is relevant to dimension, it is interesting to note that amongst all models, the more successful ones are those who inherently possess that distinct sense of scale that is somehow coherent to the scale that is unique to the process. If we are to compare models " $\mathrm{C}$ " through "F", Model " $\mathrm{D}$ " is the one model that did not produce or encompass this peculiar scale. 
(fig. 39)
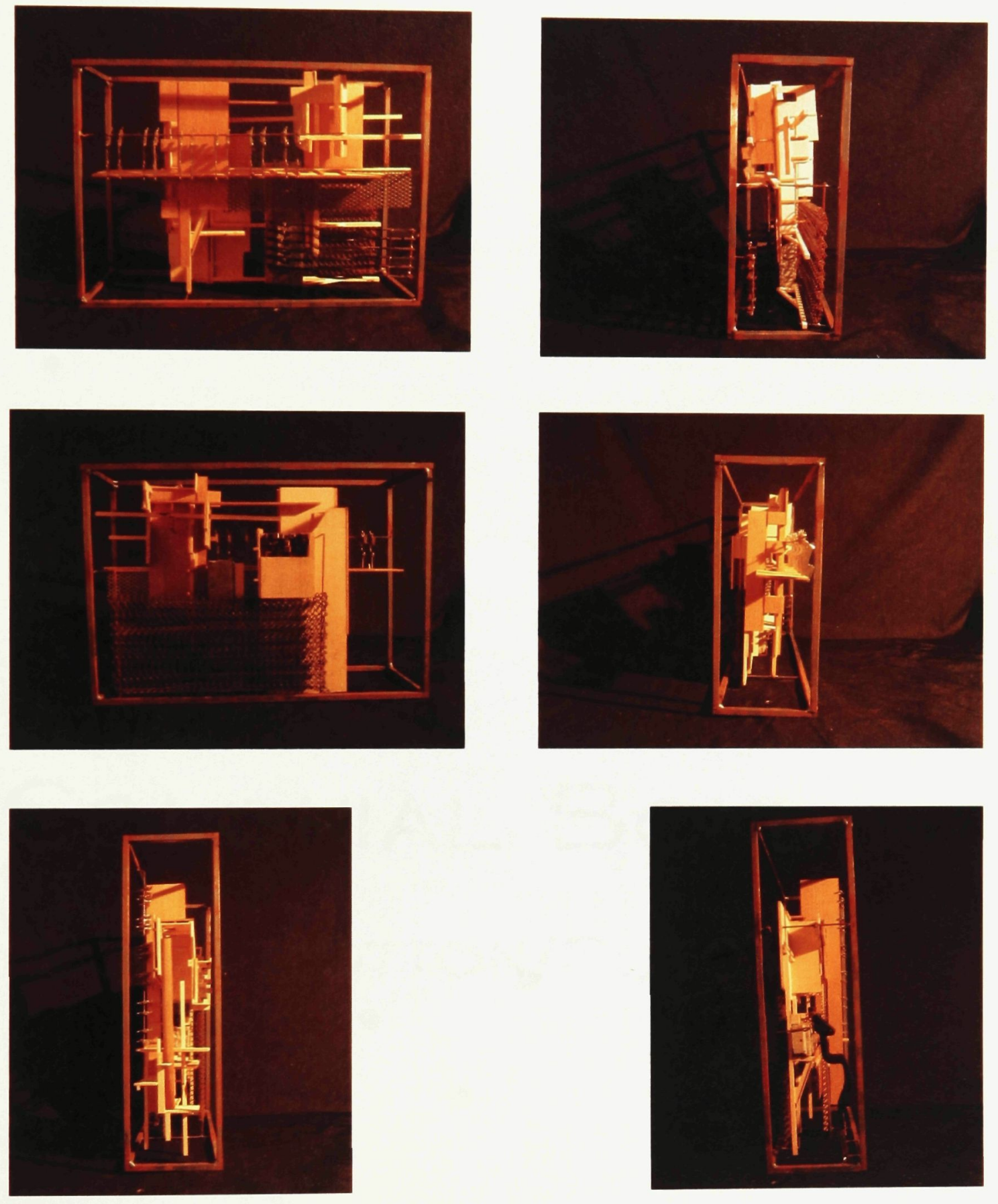
The subsequent models were a direct response to this. In fact, it was by analyzing Model "C" that highlighted a composition strategy that can avoid such a disjointed composition such as model "D". Furthermore, and apart from a disjointed composition, the importance of object selection for the model and its contextual relationship to the whole bares a striking similarity to Alberti's use of 'collocatio' in his theory of 'Concinnitas'.

'Collocatio' is the composing by place, that is, the functional placement of the details. "36 It can also be interpreted as the 'setting' or location in which the detail is reinterpreted through scale in careful consideration of its context. "The function in this case not only is limited to the practical and structural dimensions but it embodies, as well, historical and aesthetic dimensions. The placing of details, then, is deeply related to the other two requirements: Numbers and analogies. The detail in this manner is not defined by scale, but, rather, the scale is the tool for controlling it." ${ }^{, 37}$ (fig. 40)

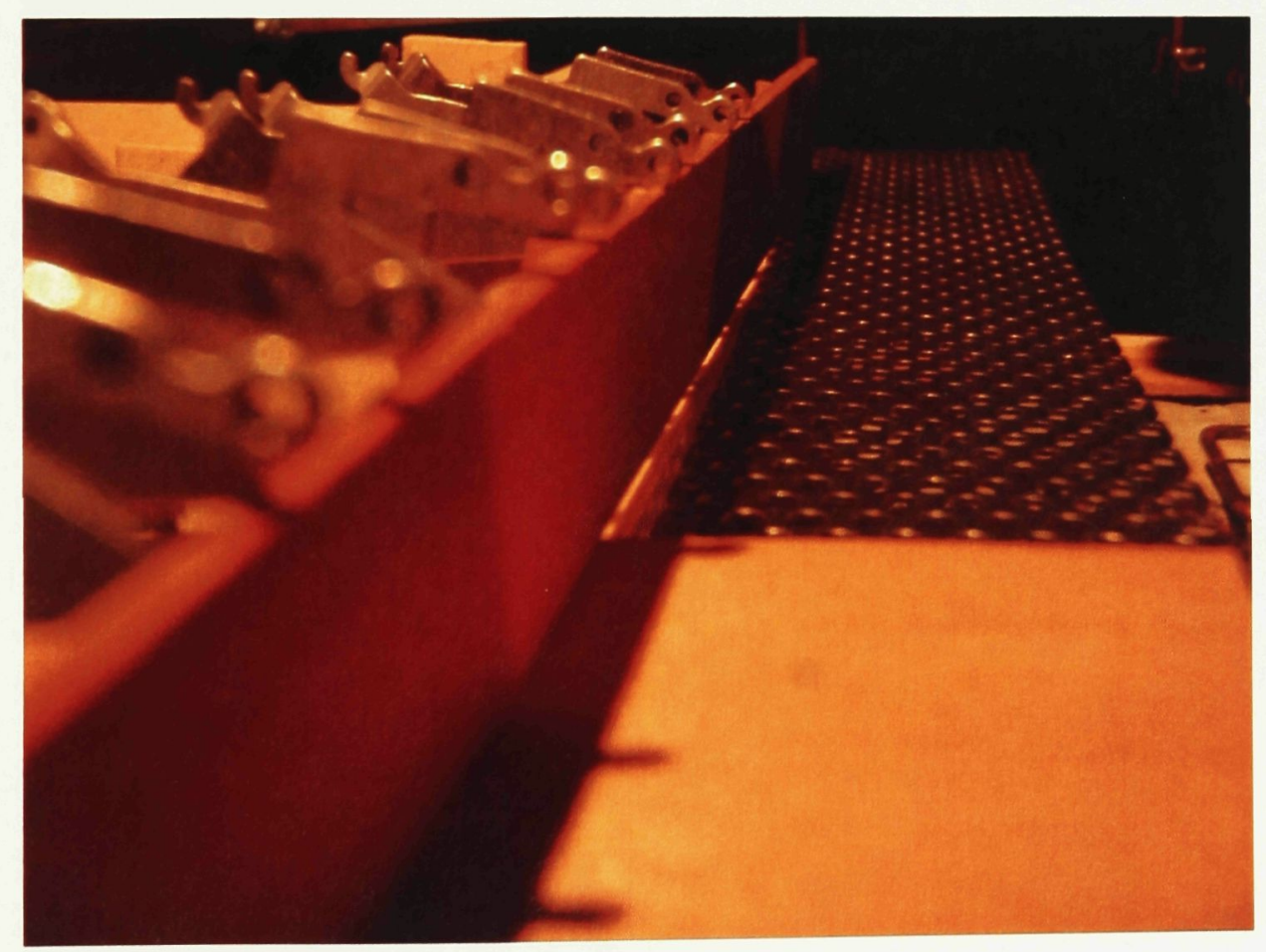

${ }^{36}$ Marco Frascari, The Tell-The-Tale Detail, Via 7, (1984), p. 27
${ }^{37}$ Marco Frascari, The Tell-The-Tale Detail, Via 7, (1984), p. 27 


\section{- Form and Space:}

The model contains a very satisfying density and occupies most of the space provided by the framework. Several of the elements point towards repetition of elements.

There is an interesting moment occurring between what seems to be a section of an exterior elevation and the intersection of a horizontal plane extending beyond the surface of this potential exterior element. This idea could offer an interesting way to approach layering; where you think something is exterior, yet becomes something interior when intersected by another space. (fig. 41)

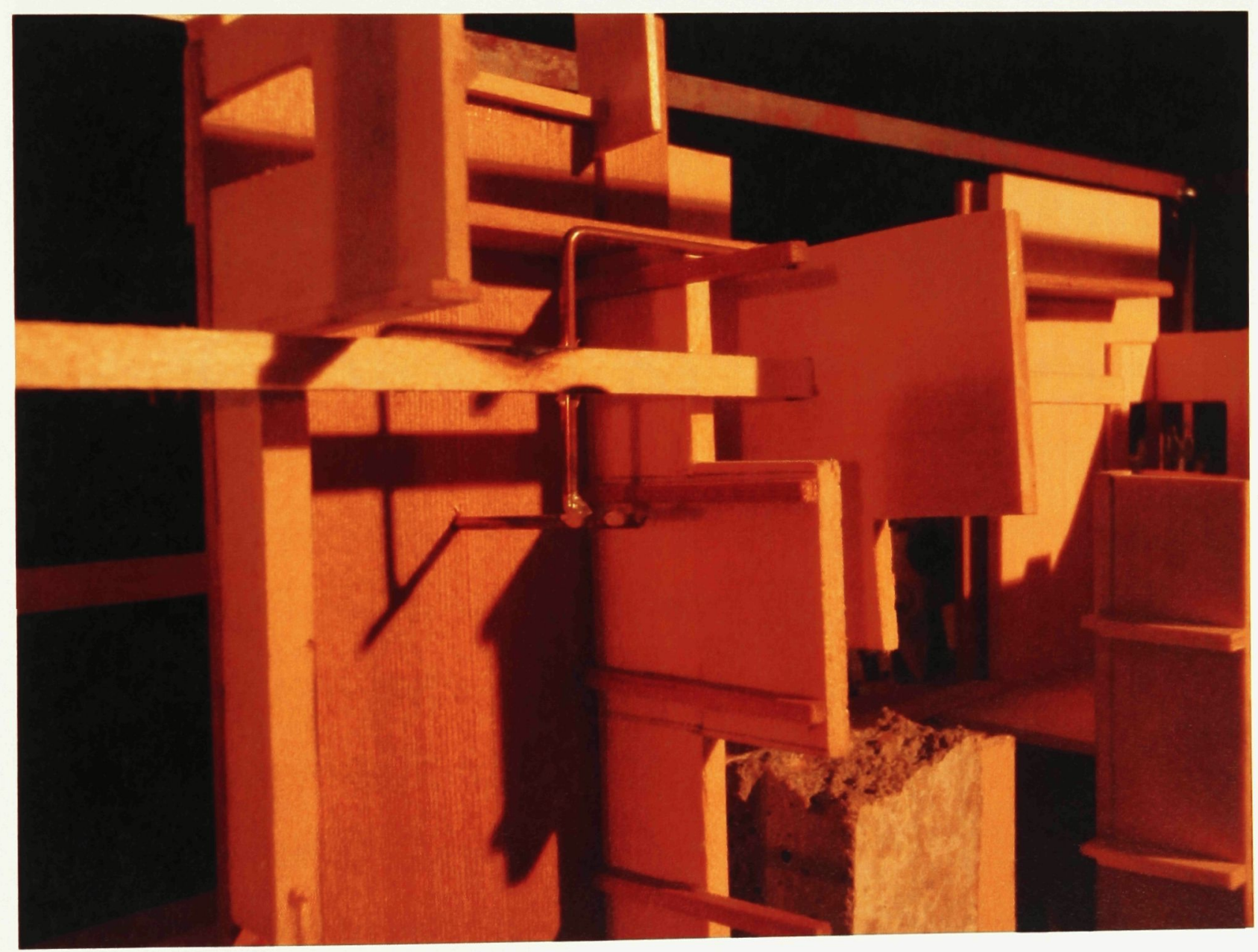


(fig. 42)

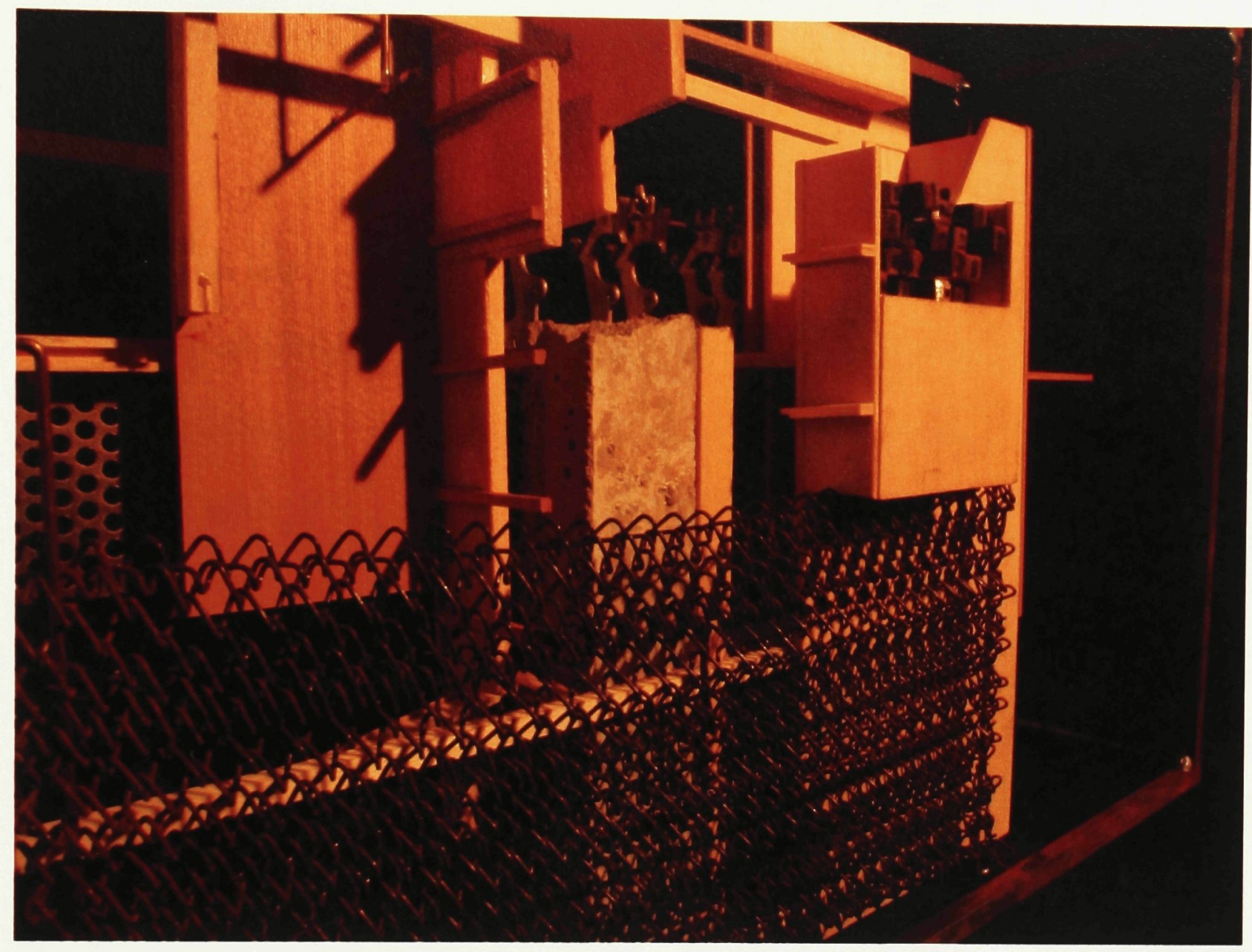

Texture is also something that is extremely present in the composition. The tactility of all the models is something that was important to the process and should be clearly present in the architecture to come. This model explores the tactile palette through the use of intricate surfaces such as chain mail, perforated sheet metal and a custom surface created from $1 / 4$ " square steel bar cutoffs and welded together. Also, we could speak of concrete and its potential to capture any surface that one can form. Some of the more articulated parts and moments are also rich spatially because we can really start imagining programmatic relationships occurring within the spaces defined. The model also suggests spaces that are disconnected from each other but connected through pathways. Planning 
this strategy may prove useful in order to get light down through the spaces of this narrow site. The typewriter parts could be used to derive an original way to support such pathways.

\section{- Limitations and Criticism:}

The use of more vibrant colours is still lacking. Perhaps for another model I would consider expanding the material palette and include synthetic material. Using the present material pallet is more faithful to the idea of basic building ingredients. This is why it is so consistent throughout all the models. (fig. 43)

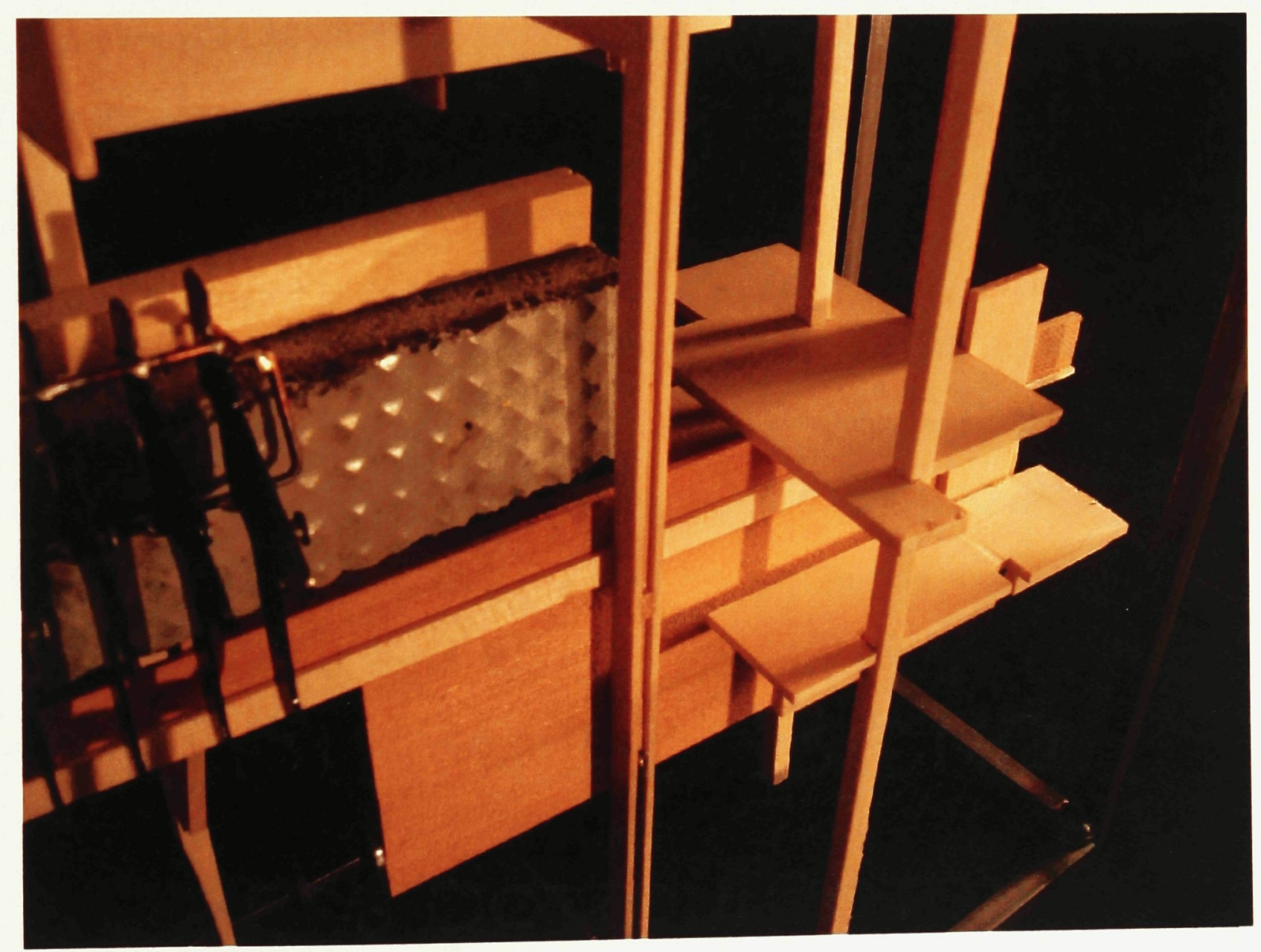




\section{TRANSLATION FROM MODEL TO ARCHITECTURE}

Rather than describing the traditional walkthrough of the architectural project, this chapter is dedicated to the translation of the models into architectural form. It is necessary to understand that the model as a whole should not be literally and formally interpreted into architecture. Rather it is the moments, parts, spaces, details, surfaces, and materials that should be reinterpreted and translated architecturally. Since the program is restricted to a narrow and challenging site, it is important for the street façade to be engaging and maintain a subtle presence amongst the existing urban fabric. Model ' $F$ ' offers a very rich solution in terms of defining the parameters for the articulation of the Bank street elevation.

The sectional aspects of model ' $F$ ' and its complex formal and spatial condition, initiated the general spatial organization of the project. The most prominent spatial condition occurs where the horizontal plane intersects the vertical element. In this case, the vertical element is interpreted as a building envelope intersected by a floor plane. This condition, brought forth by model ' $\mathrm{F}$ ', prompted the consideration of designing the elevation by means of layering. Based on this spatial condition, a series of layers were developed to inspire the articulation of the Bank street façade. The idea was to create several building layers which would blur the exterior threshold. The first skin is opaque and includes generous openings to allow natural light to penetrate. The second skin is mostly glazing and is contrasted by an adjacent vertical section of masonry. The condition changes on the 
ground floor to accommodate the commercial programming. The elevation is recessed from the consistent street façade for several reasons. The first is to create a more substantial and hence, a more engaging entry sequence. Secondly, it interrupts the consistent architectural rhythm of the street giving the new architecture more presence in the existing context. Furthermore, given the nature of the program, it seems appropriate to allocate an exterior space for a ground floor terrace for use in warmer seasons. The initial elevation was further developed through an axonometric study, while simultaneously

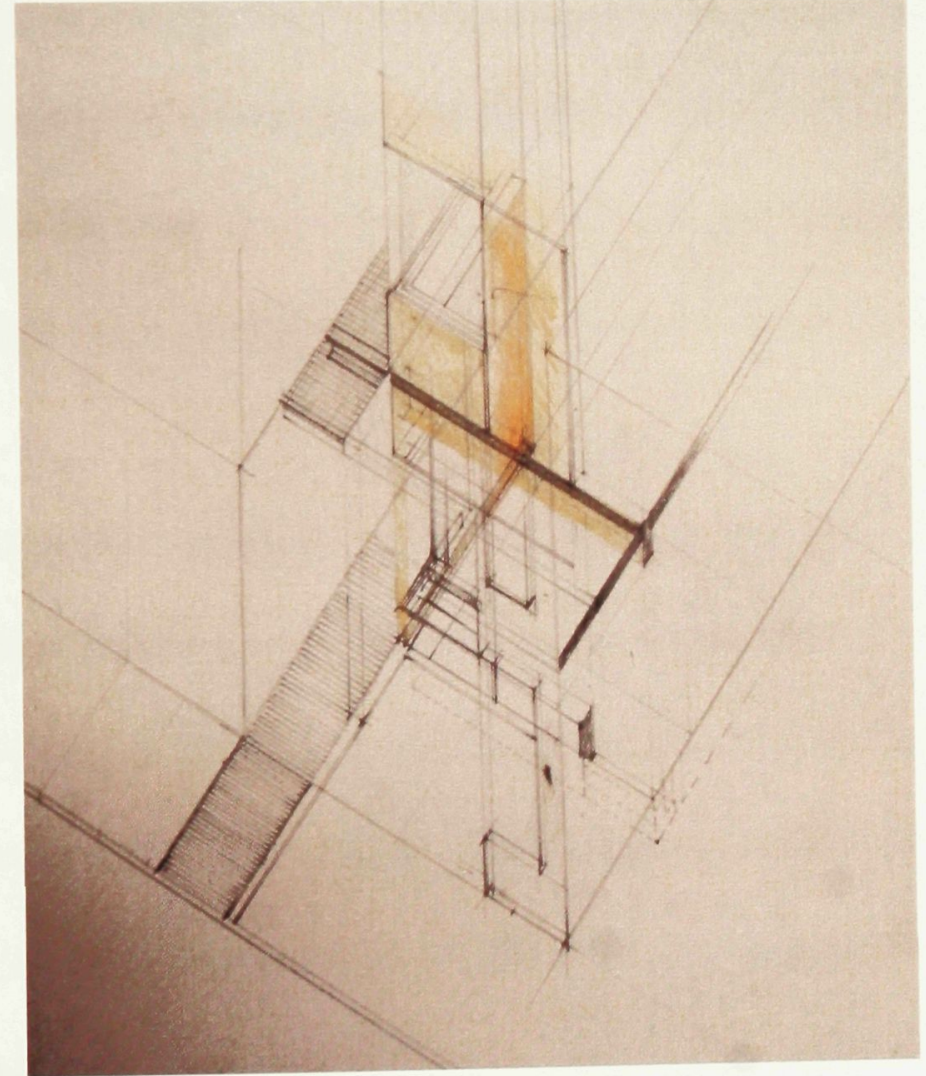
considering its materiality. (fig. 44) Once the elevation was roughly laid out, initial floors plans and sectional studies were generated in relationship to the program.

On the ground floor, we find a space for sale purposes adjacent to a central customer seating area that is deliberately recessed to maintain a visual relationship from the sales and display

counter, to the street and its pedestrians. The area located behind the sales area is designated for services and vertical circulation. 
The second floor is where the baking and dressing of pastries occurs. At the street side of this floor, a workstation is located between the dual skins, for the dressing and finishing of cakes and pastries. The juxtaposition between the glass and brick is a reinterpretation of a traditional pastry display case. Exposing this task of finishing to the public street considers the act of making as a performance. It creates a reciprocal viewing relationship between the street and the users of the architecture. The main space of this level is where the production of pastries occurs. The kitchen contains the necessary equipment for this particular culinary art. The storage of daily stock is located at the rear of the second floor next to the stairs. This space contains dry and refrigerated ingredients for daily use. The third floor houses a small administrative office. The office space partially projects into the three storey open space to allow for communication between the floors. The large main space of this third floor is dedicated to any preparation work.

An added challenge was to somehow experientially engage both the users and the clients phenomenologically throughout the architecture. The width of the site and the layering of the programming is a challenge because it limits the programmatic interaction that can occur on the same plane. The alternative was to consider the spatial relationships in sections and analyze the overlaps occurring programmatically. The objective is to engage the users through their senses. Some of the materials present in the models offer potential solutions that allow these phenomenological conditions to take place.

On the second level, the main circulation paths are composed of steel grating. This particular material has been chosen specifically because of its physical properties - - It is 
strong and robust, yet extremely porous and open. This material transforms into a filter that allows certain things to penetrate through but not others. Sounds, shadows, light, and aromas, flow through the steel grating while supporting the functions above. Using these senses as a means of establishing spatial relationships through this narrow building strengthens the experience of the architecture and the program.

Since the site is so narrow, it is important to consider the presence of natural lighting within the architecture. Materials composing the front and the rear elevation allow for the penetration of a considerable amount of light. The challenge lies at the center of the building where light struggles to reach its surfaces. This problem is resolved by the introduction of a three-storey light well, drawing an abundance of natural light in the heart of the building. The porosity of the circulation paths that extends through this tall open space allows the light to reach the first level. The large vertical surface that reaches through the core of the building is influenced primarily by the concrete element found in model 'B'. It was reinterpreted into a more refined assembly. Composed of pre-cast concrete elements of varying lengths connected by thick steel brackets, this assembled wall would become a massive yet porous textured surface.

The idea of texture and colour is something that is present in all models. In many cases, texture is also key to the success of pastries. Furthermore, textures should also maintain a strong presence in the proposed architecture. A diversity of rich materials, such as: glass, wood, clay brick, glazed tile, bronzed copper, steel, and concrete, offer such textures to this project; The models directly inform this material pallet. 
These architectural models can provide the viewer with innovative detailing opportunities by simply perusing through the models. It is a simple question of observing and imagining these moments, which may then be translated by the interpreter through diverse methods. It may require a few attempts accompanied by some graphic investigations to elaborate some of these details, but nonetheless, these details are explicitly generated from these models. Details such as handrail connections, the door at the main entrance, the window leavers in the kitchen, the seats in the pit area, are some of the more apparent examples that were drawn from the models. (fig. 45)

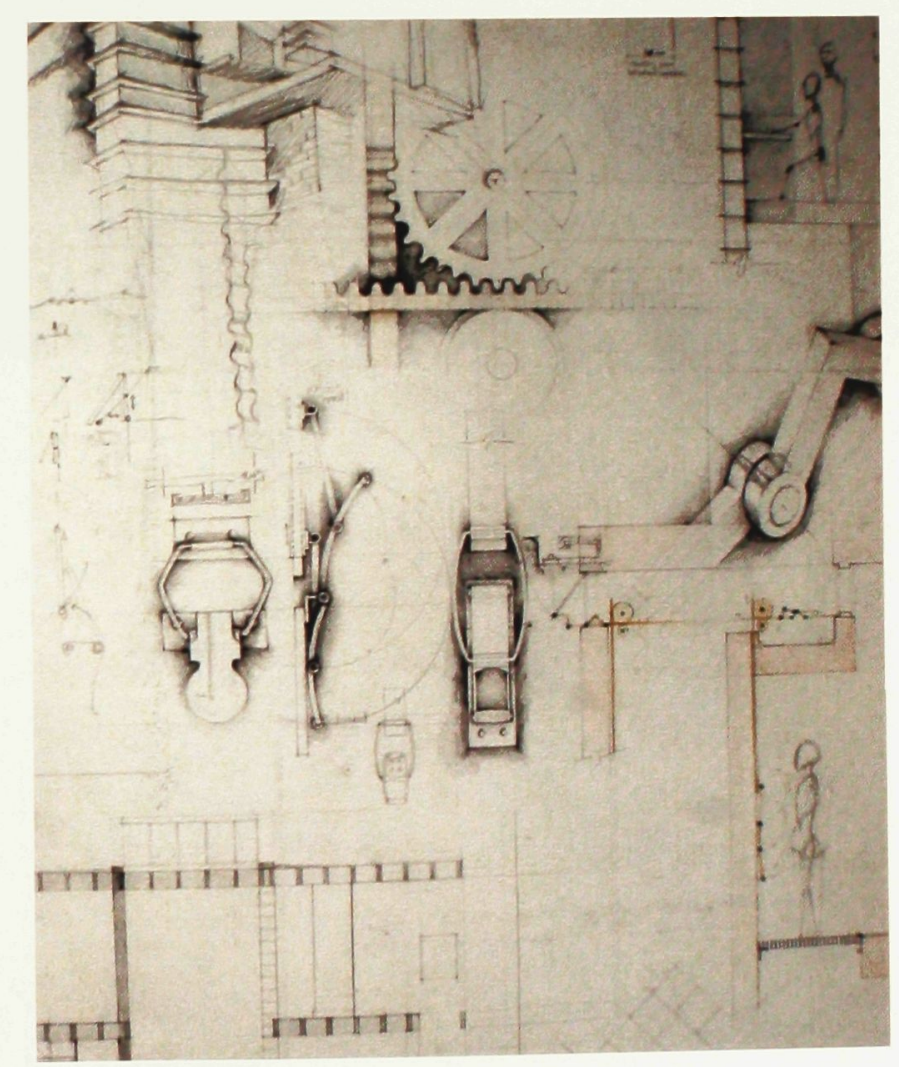


Fig. 46

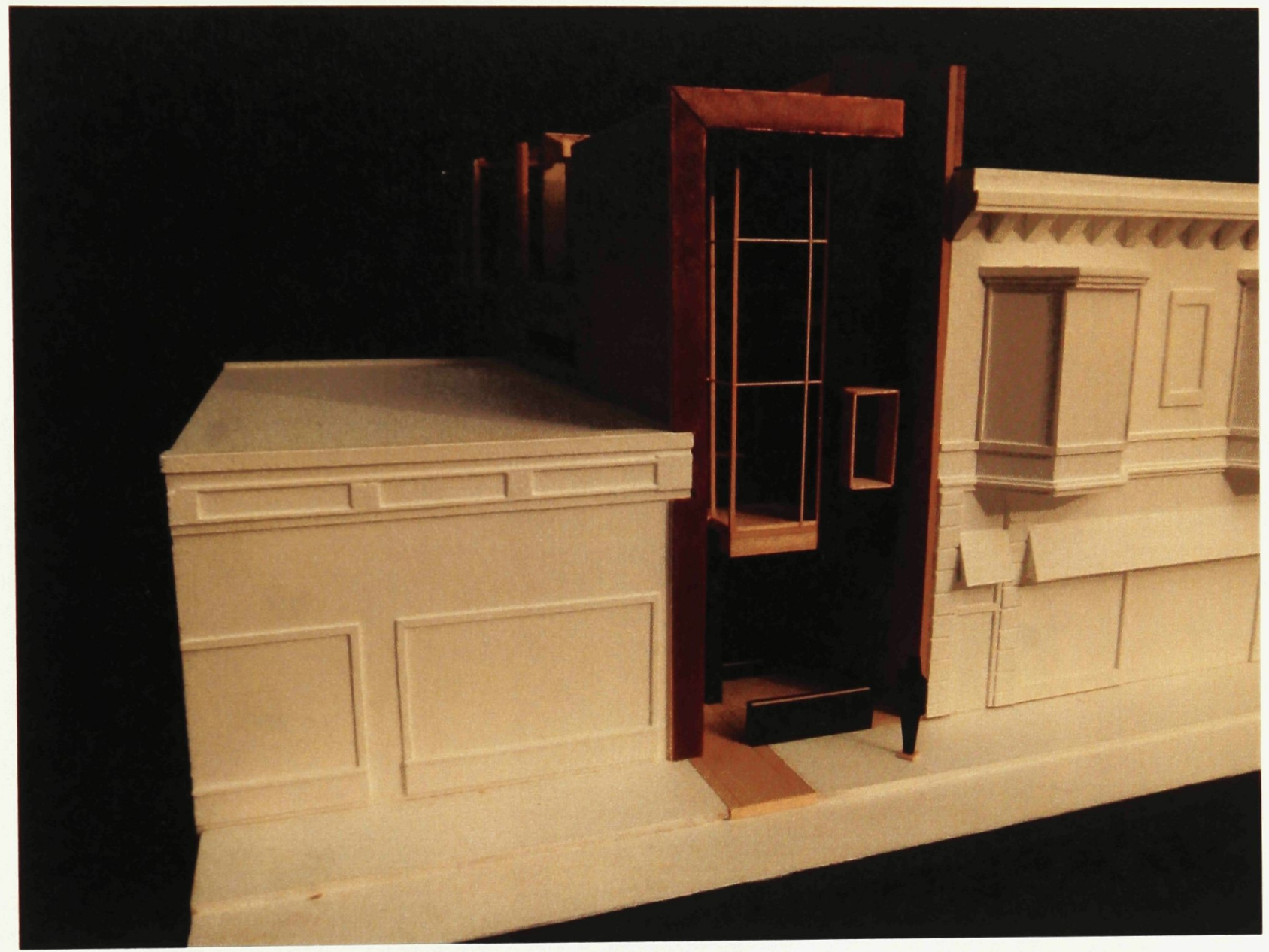


Fig. 47

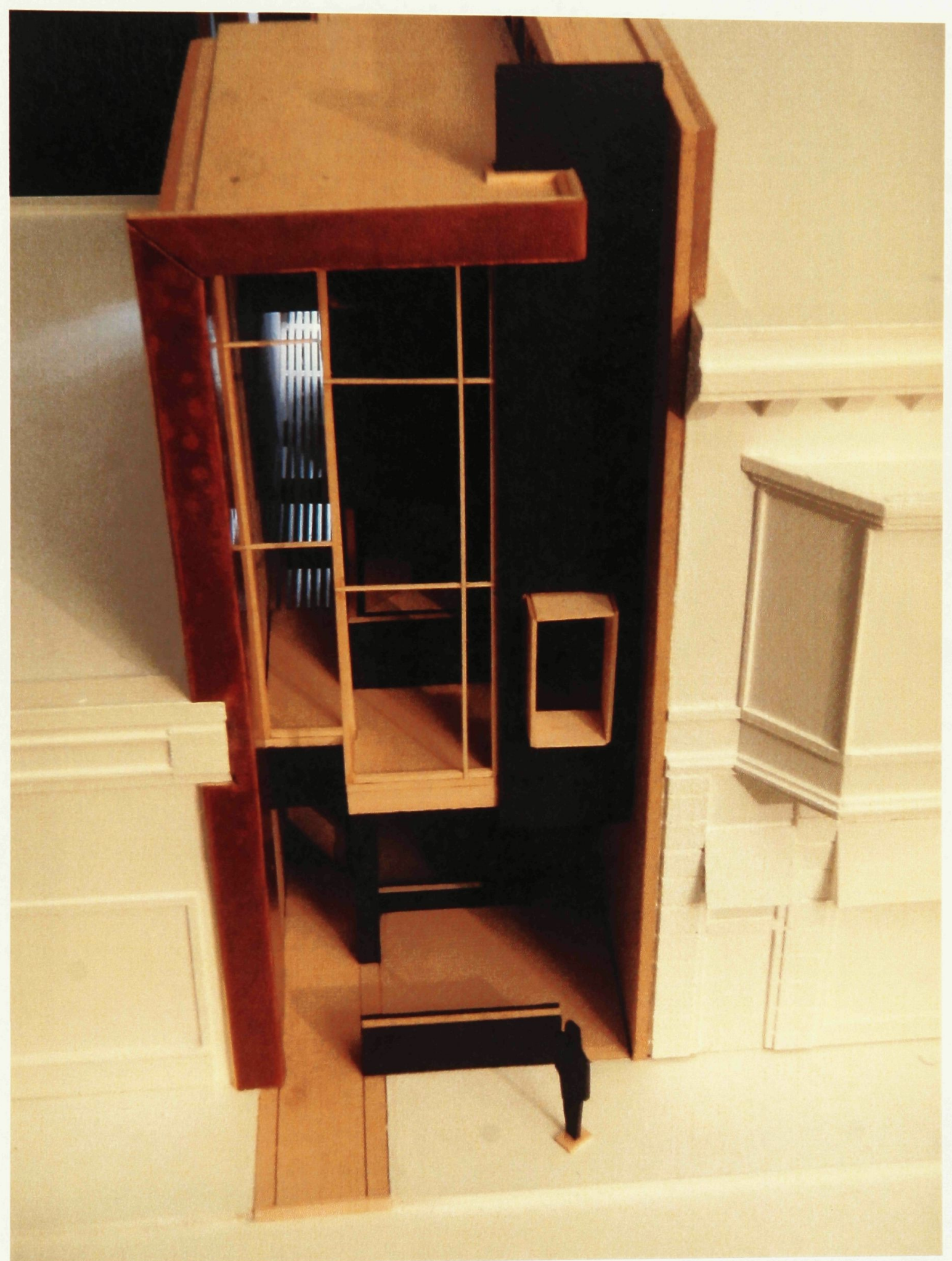


Fig. 48

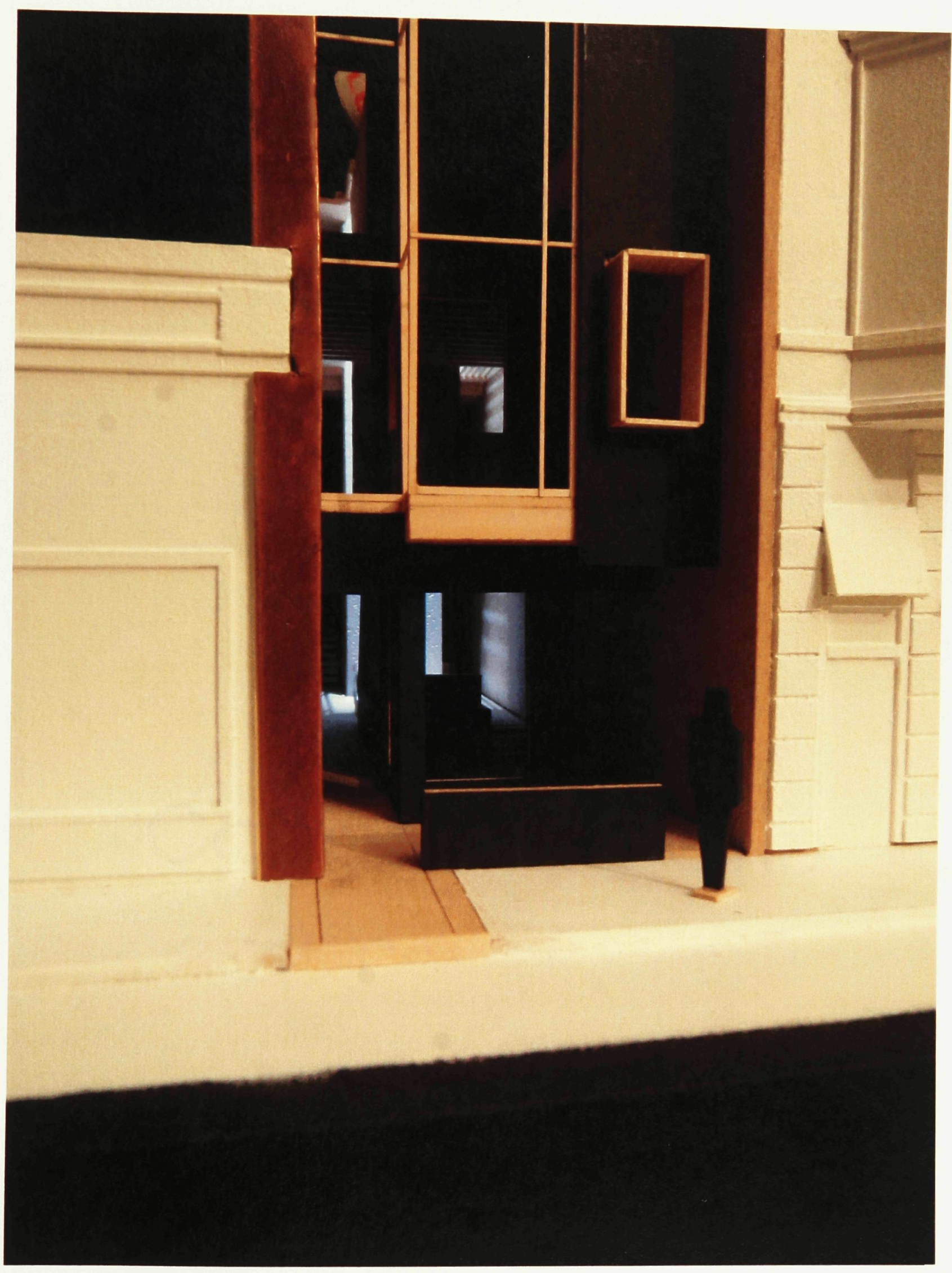


Fig. 49

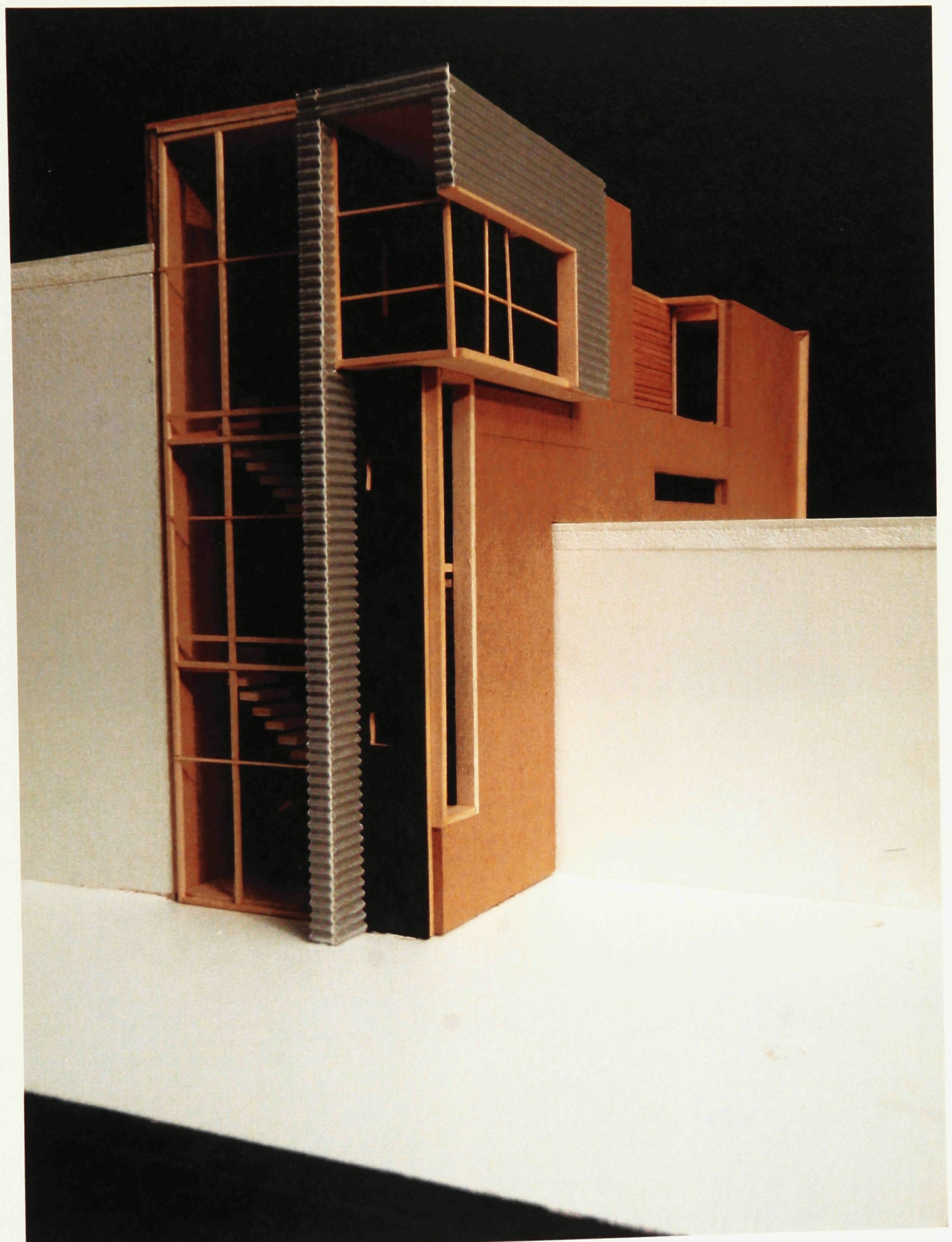


Fig. 50

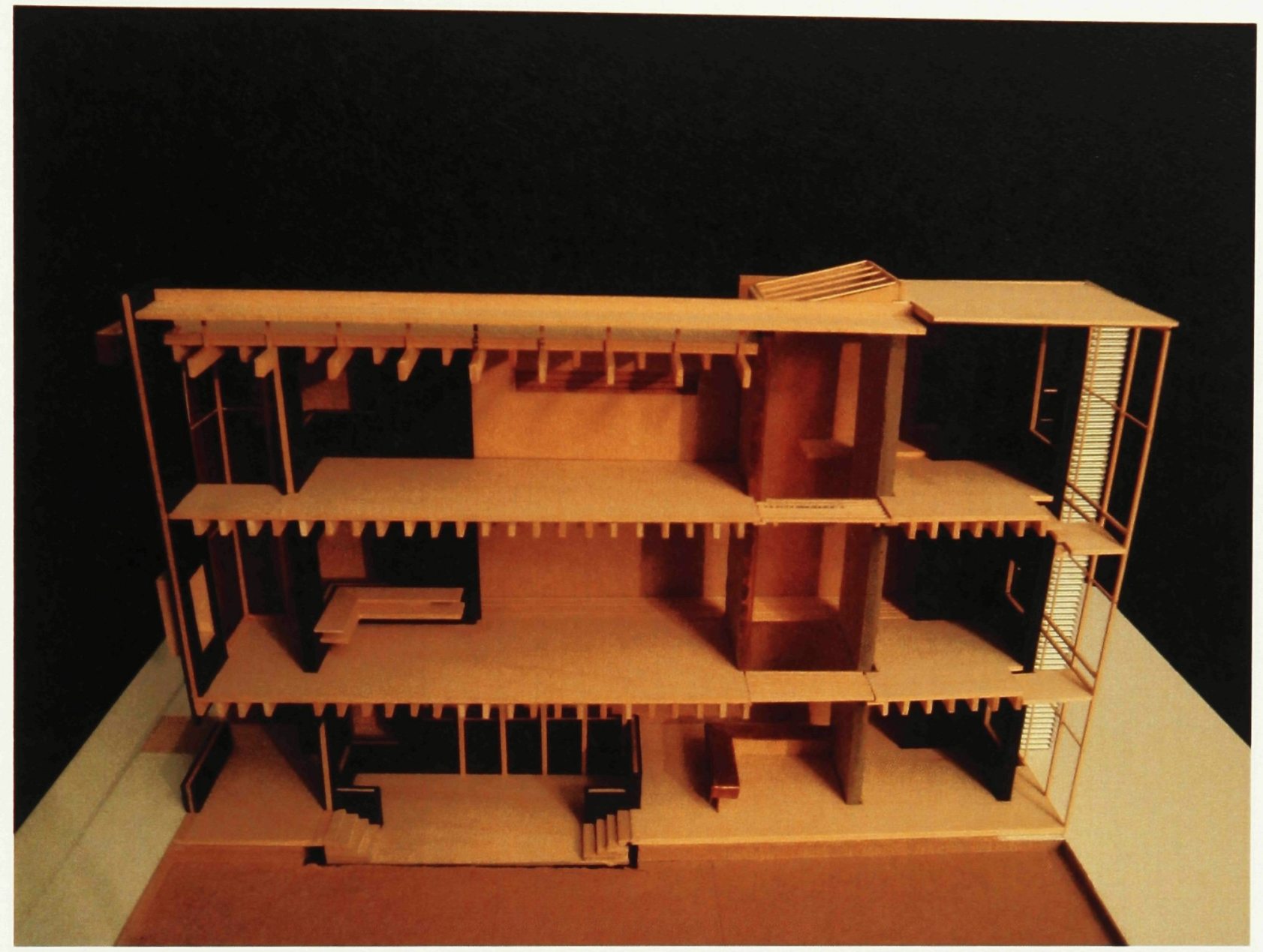


Fig. 51

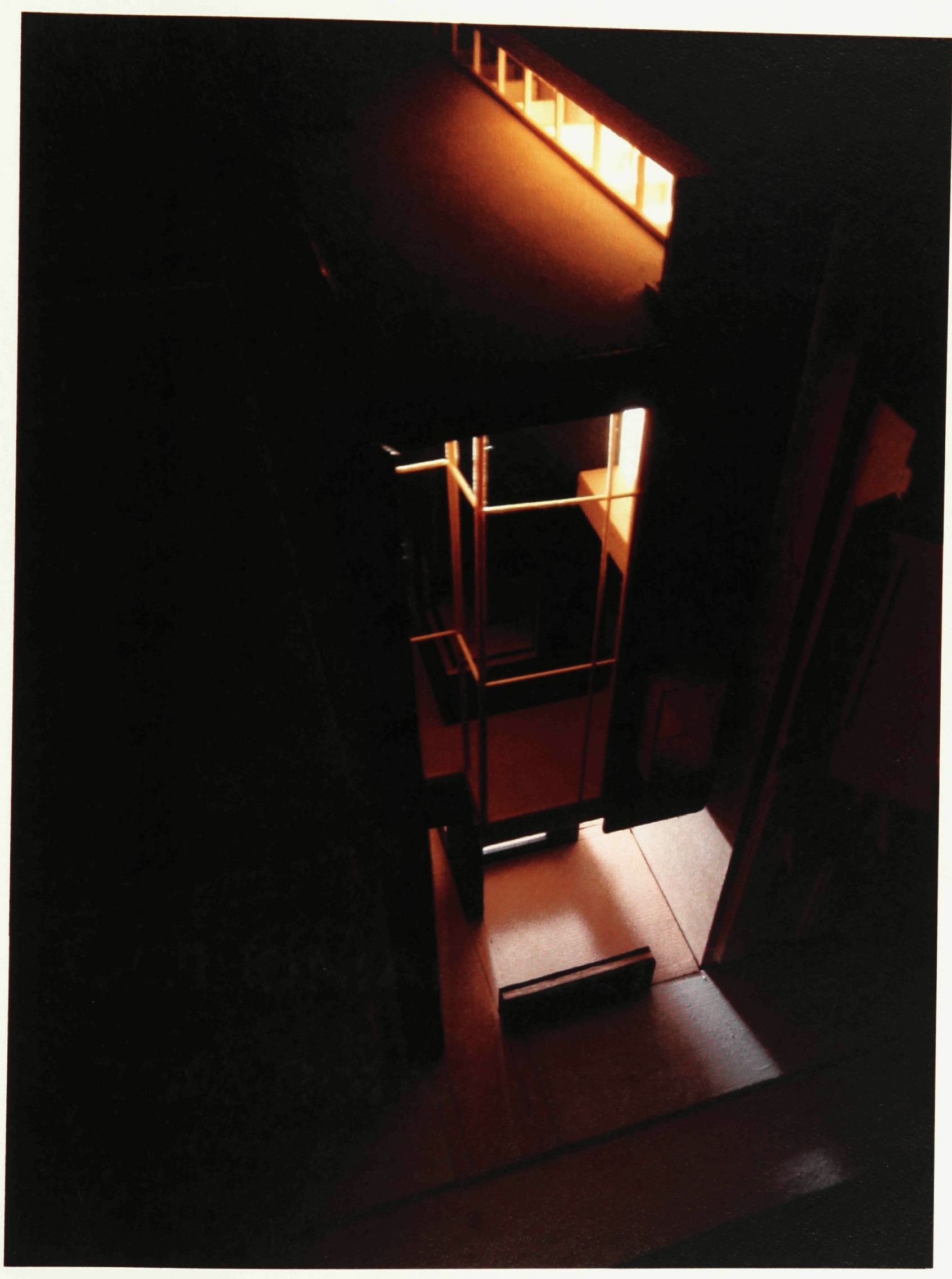




\section{METHOD OF GRAPHIC PRESENTATION}

The graphic means of presentation for this thesis explores an alternative to the traditional mode of architectural representation. The enclosed architectural drawings share a significant relationship to the process. Due to the communicative and demonstrative nature of the models, it was necessary to express the project in a graphic mode that complemented these qualities. The drawings produced are intended to be demonstrative in nature and to show intent, process, and outcome, rather than the typical 'presentation' drawings which typically emphasize an end result. (See appendix for complete drawings)

Carlo Scarpa and Mario Ridolfi are significantly important in regards to the methodology of demonstrative drawings. "Representation as demonstration is at the basis of the professional services of Scarpa and Ridolfi. Both of these architects show a concern with representing and devising construction through an appropriate use of representation. On the one hand, the art of their drawing does not belong to the present fad of fanciful, publishable presentation/design drawing. On the other hand, there is no reason to mistake their graphic elaborations for the pure, axiomatic, perfect professional work of a skilful working drawing." 38

\footnotetext{
${ }^{38}$ Marco Frascari, Monsters of Architecture, p. 100
} 
These intricate drawings developed by both architects demonstrate a technological narrative of construction. The drawings are not to be read as definitions, rather, they should be interpreted as informal instructions. "Another characteristic of these drawings is the almost constant written comment, which is not only numerical and descriptive, but self critical, ironic, and playful, without even becoming deceptive or ambiguous. It is architectural calligraphy, a beautiful writing of construction tropes, which is concerned with the forming of an uncommon figure of drawing." 39

Although their graphic techniques differ, the outcome of their rich drawings is quite similar. Scarpa develops his architectural intentions on Bristol board that is then overlaid with light tracing paper. Drafting and coloured pencils, diluted ink, and "Pentimenti" are prominent features of his drawings. (fig. 52-53) 'Pentimenti' is a technique that uses an underlying image in a painting, a part of a painting, or an original draft, which is brought forth through the top layer of paint. Ridolfi, however, draws on thick tracing paper (carta de lucido), and uses the fountain pen as his medium of preference. He edits his drawings through skillful use of scissors and adhesive tape. "Scarpa and Ridolfi's technographies are marvelous ways of writing architecture. They are wonderful calligrams of technological thought, and the analogical expression of the process of contruction. They are visual descriptions of processes that are not visible. They are conceived not to be read by the public, but rather to carry out a demonstration of intent." ${ }^{, 40}$ (fig. 54-55)

Fig. 52

${ }^{39}$ Marco Frascari, Monsters of Architecture, p. 100

${ }^{40}$ Marco Frascari, Monsters of Architecture, p. 102 

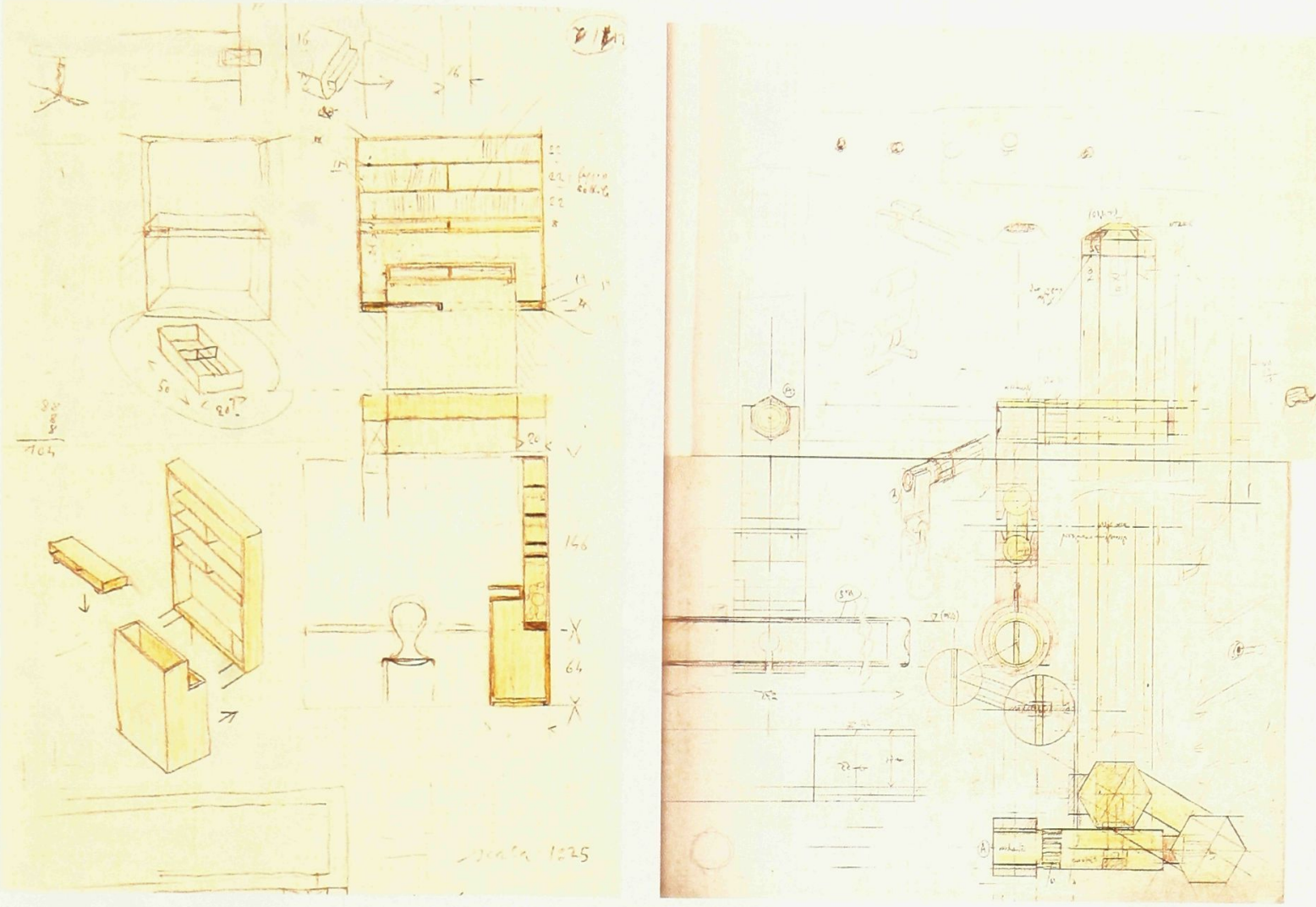

The traditional methods of architectural graphic representation are generally concerned with displaying the future physical condition of the architecture. Furthermore, "their Cartesian rigor deals only with the visible side of technology; technographies deal instead with the invisible side, making tangible the real measure of architecture." 41 The premise behind using this alternative mode of representation is to underline the process of production rather than showing the end result, the product. Representation as demonstration requires architectural mediation, which in turn allows the architect to be self-critical. This process becomes a continuous dialogue between drawing and architect. Fig. 54

${ }^{41}$ Marco Frascari, Monsters of Architecture, p. 102 


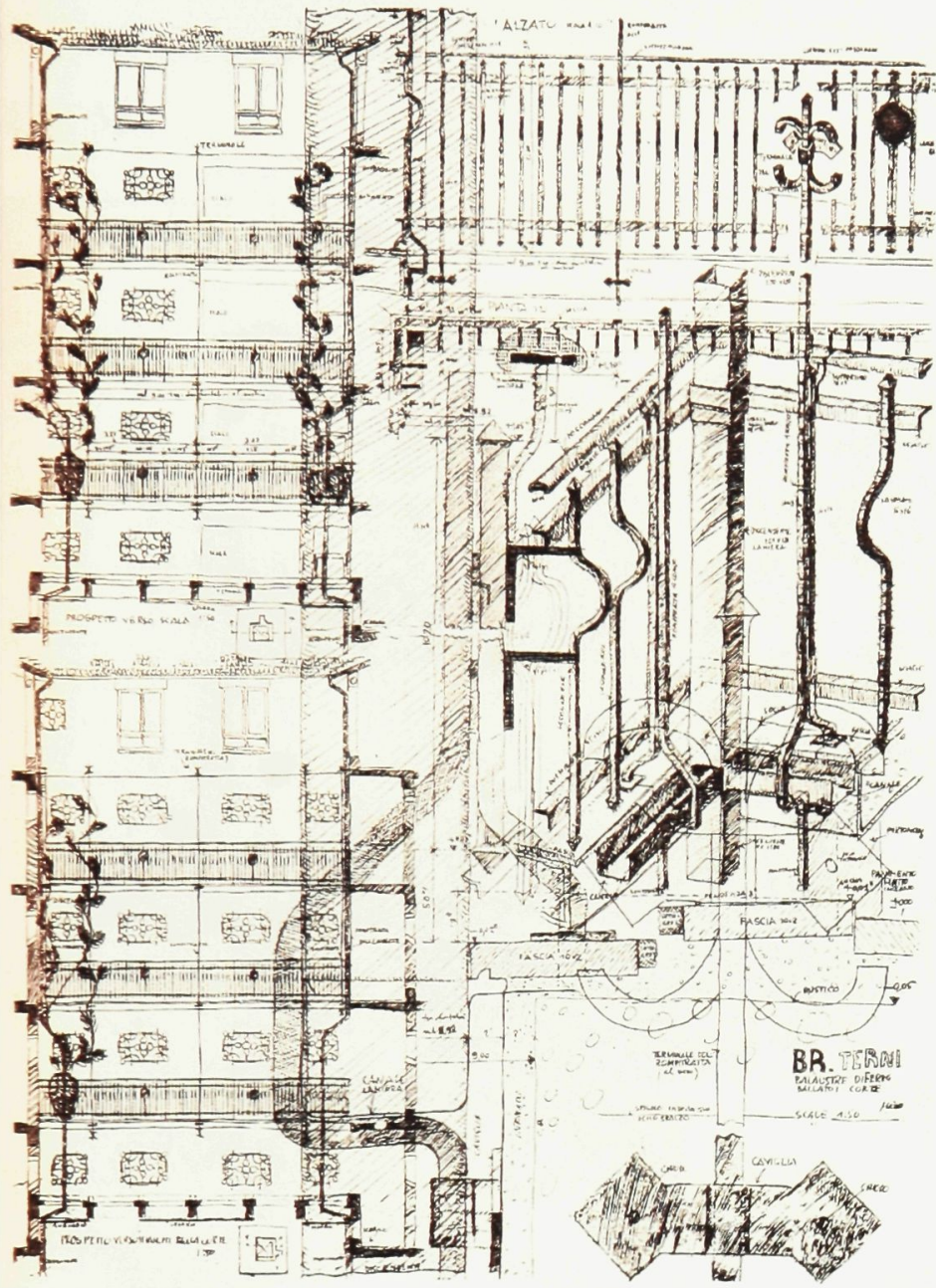

Similarly, these analogical models seek to provoke this dialogue during the process of production. Producing such models cannot occur without understanding the reciprocal relationship that occurs during the process of making. It is a conversation between model and maker. 
Fig. 55

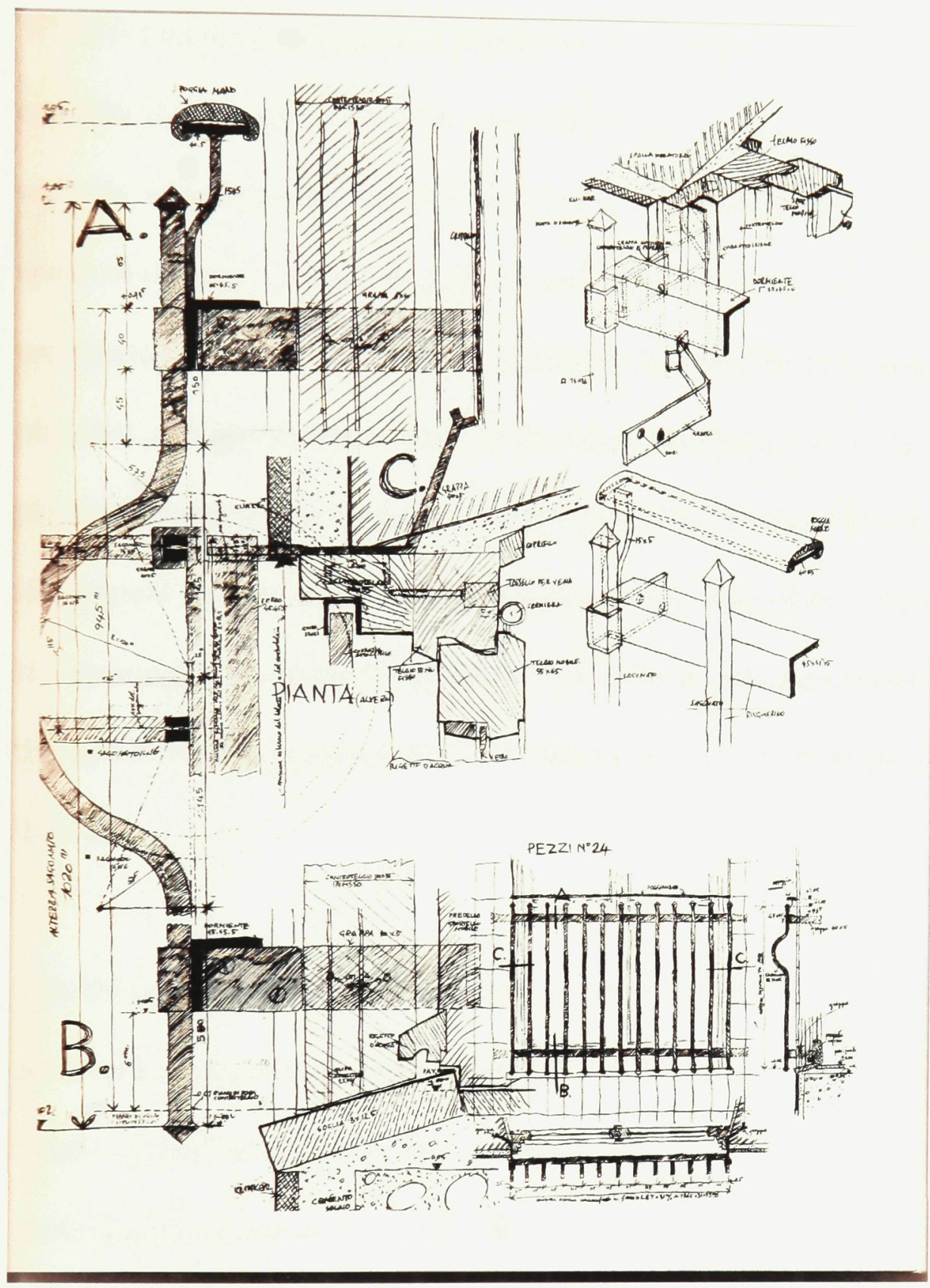




\section{CONCLUSION}

When we explore the physical conditions that compose architecture, we must carefully consider the medium we utilize when investigating these phenomenological conditions. If we truly wish to pursue the physical dimensions and limitations of architecture, whether of space, form, or materials, why do we insist on exploring these physical moments of architecture on a two-dimensional surface? Challenging these physical issues in a tangible, physical, and immediate system of 'physical sketching' immediately embodies the physical characteristics innate to architecture. The intent of the thesis is not to criticize the ideological validity of Lissitzky's prouns, or the basis of the 'analytique' drawings, rather, it is to suggest that their principles of space, form, and materiality should be expressed through a more appropriate medium; through the use of analogical models as described therein.

This thesis has, based on the outcome of the process developed, achieved its objective: To develop a physical analogical model that has the potential of inspiring architectural creativity, and that has not been preconceived prior to its making. Through the evolutionary nature of the modeling process in relationship to the technique of collage, unexpected discoveries were made. Since there were no prior instructions on how to make a successful analogical model, the process itself developed its own necessary guidelines in respect to scale, materiality, composition, and appropriate use of found objects. Inspired by Marcel Duchamp's piece, 'Étant donnés', using this scientific cataloging of comments 
and observations, proved very useful to the development of the process. This Logbook provided the underlying framework for this self critical process to evolve.

Initially, it was assumed that the process for such a generative model would deliver one all-encompassing model. However, once the modeling process was finished, a major dilemma occurred - - Should a singular model be used as a source of creativity, or should they all be tools from which we draw elements to generate the architecture? Due to the personal involvement in the fabrication of the models, it is extremely difficult to ignore many particular and fascinating moments occurring during their construction, making it extremely hard to eliminate these as a source of formal ideas. Since the models had been developed for the same purpose, all were considered in the development of the final project.

These types of models can be employed for many projects. However, variables such as site, context, program, and dimensions, will change the outcome. For this reason, it is crucial to develop these models in a series, which in turn forces the refinement of the models as they evolve. Also, when you generate a series of models, it is important that they share common elements of reference. Doing so creates a basis for comparison and allows for a more accurate analysis. For this thesis, the consistent materials selected, and the steel framework (for models C-F) provided these crucial elements of reference.

Furthermore, the scientific logbook forced a consistent re-evaluation of the models that helped to inform and permit the evolution of the next generation of models. 
The creation of an architectural model that generates architectural creativity, possibilities, and solicits multiple interpretations is a reaction to the socio-cultural conditions under which we operate. Presently, North American architectural practices are adamantly bound to building codes and construction standards which unfortunately offer architects simple solutions. This trend of pre-conceived solutions has the potential to cripple our architects and our profession. We only need to look at the latest architecture that surrounds us to see in what unfortunate direction our profession is headed. These so called standards eliminate the opportunity for architects to think and resolve architectural issues, whatever they may be. Thinking about problems entices us to imagine solutions, and it is through imagination that we discover innovations. The models that this thesis proposes should force us to engage architecture thoughtfully both in its parts and its entirety. Reflection, imagination, and innovation are exactly what these models seek to provoke in the observer: to stray from the obvious standards of our profession and encourage architectural creativity. However, pursuing these innovations requires profound knowledge of fundamental architectural materials. Mastering these fundamentals principles allows us to engage architecture with more creativity and flexibility. How far could our creative minds chase our architectural fantasies if they were free from the rule of building codes and standardized construction methods? 


\section{BIBLIOGRAPHY}

Smith, C. Albert, ARCHITECTURAL MODEL AS MACHINES, A New View of

Models from Antiquity to the Present Day, Architectural Press, Elsevier Ltd. 2004

Adamowicz, Elza, SURREALIST COLLAGE IN TEXT AND IMAGE, Dissecting the

Exquisite Corps. Cambridge University Press, 1998

Breton, André, NADJA, Grove Press Inc. New York, 1960

Benjamin, Andrew, Re: Working Eisenman, Academy Editions - Ernst and Sohn. 1993.

Spies, Werner, MAX ERNST Life and Work, Thames \& Hudson, 2005

Frampton, Kenneth, STUDIES IN TECHTONIC CULTURE: The Poetics of Construction in Nineteenth and Twentieth Century Architecture. Massachusetts Institute of Technology (MIT Press), 1995

Evans, Robin. TRANSALTING FROM DRAWING TO BUILDINGS AND OTHER ESSAYS, Architectural Association London.

Zumthor, Peter. TEACHING ARCHITECTURE, Lars Mueller Publisher, 1998

Weston, Richard. MATERIALS, FORM AND ARCHITECTURE, Yale University Press, New Haven, CT, 2003

Robbins, Edward. WHY ARCHITECTS DRAW, Massachusetts Institute of Technology (MIT Press), 1994

Cellini, Francesco, And, D'Amato, Claudio, LE ARCHITETTURE DI RIDOLFI E FRANKL, Mondadori Electa, S.p.A, 2005

Murphy, Richard. ARCHITECTURE IN DETAIL: Querini Stampalia Foundation; Carlo Scarpa. Phaidon Press Limited. 1993 
Frascari, Marco.

THE TELL-THE TALE DETAIL,In: Via, No. 4, (1984) :2237

MONSTERS OF ARCHITECTURE: Anthropomorphism in Architectural Theory. Princeton, NJ: Rowman and Littlefield, 1991.

Pallasmaa, Juhani.

-EYES OF THE SKIN: Architecture and the Senses, ACADEMY GROUP LTD. 1996

- AN ARCHITECTURE OF THE SEVEN SENSES. Japan Architecture and Urbanism, 1994 
$\underline{\text { APPENDIX }}$

Architectural Drawings 


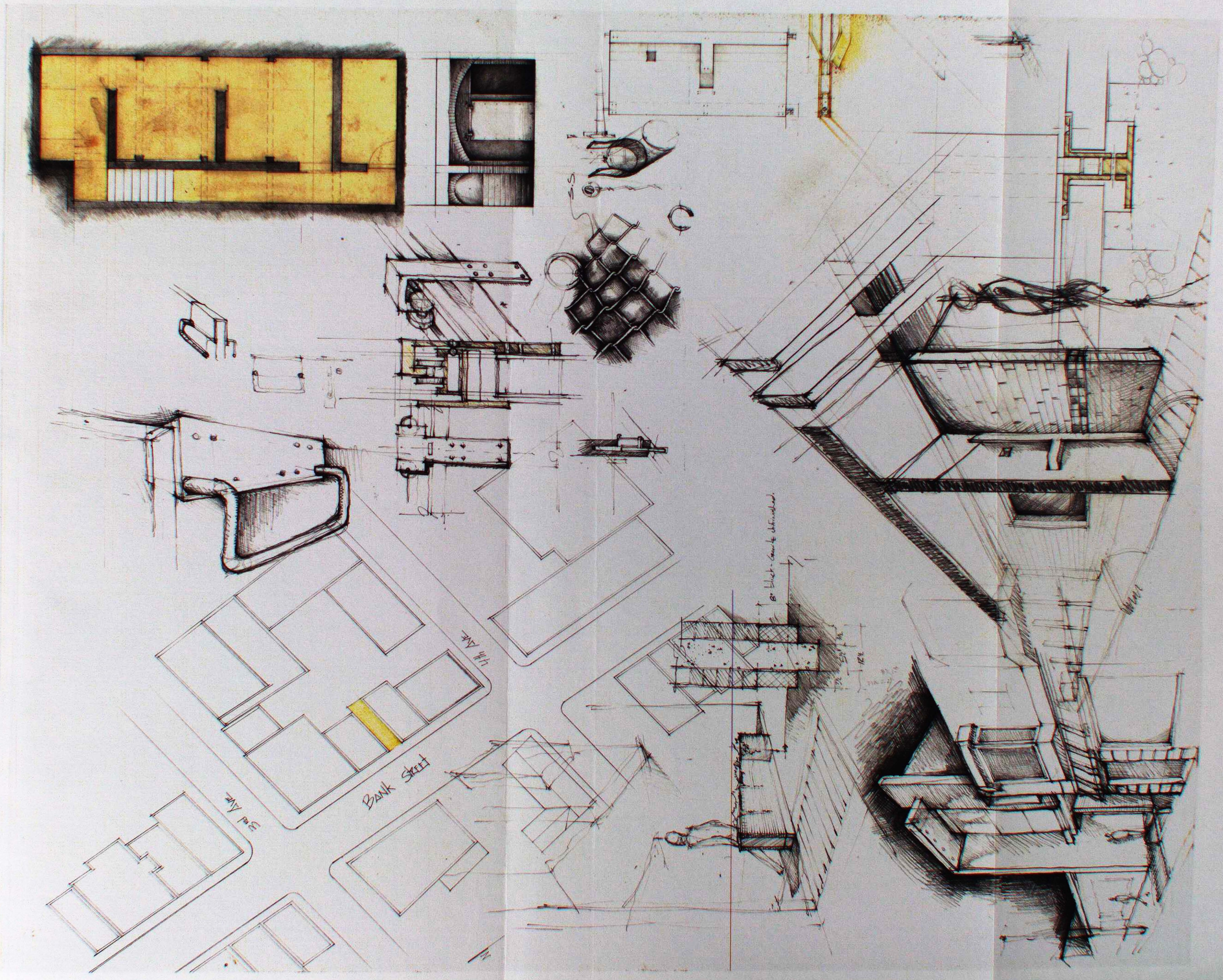




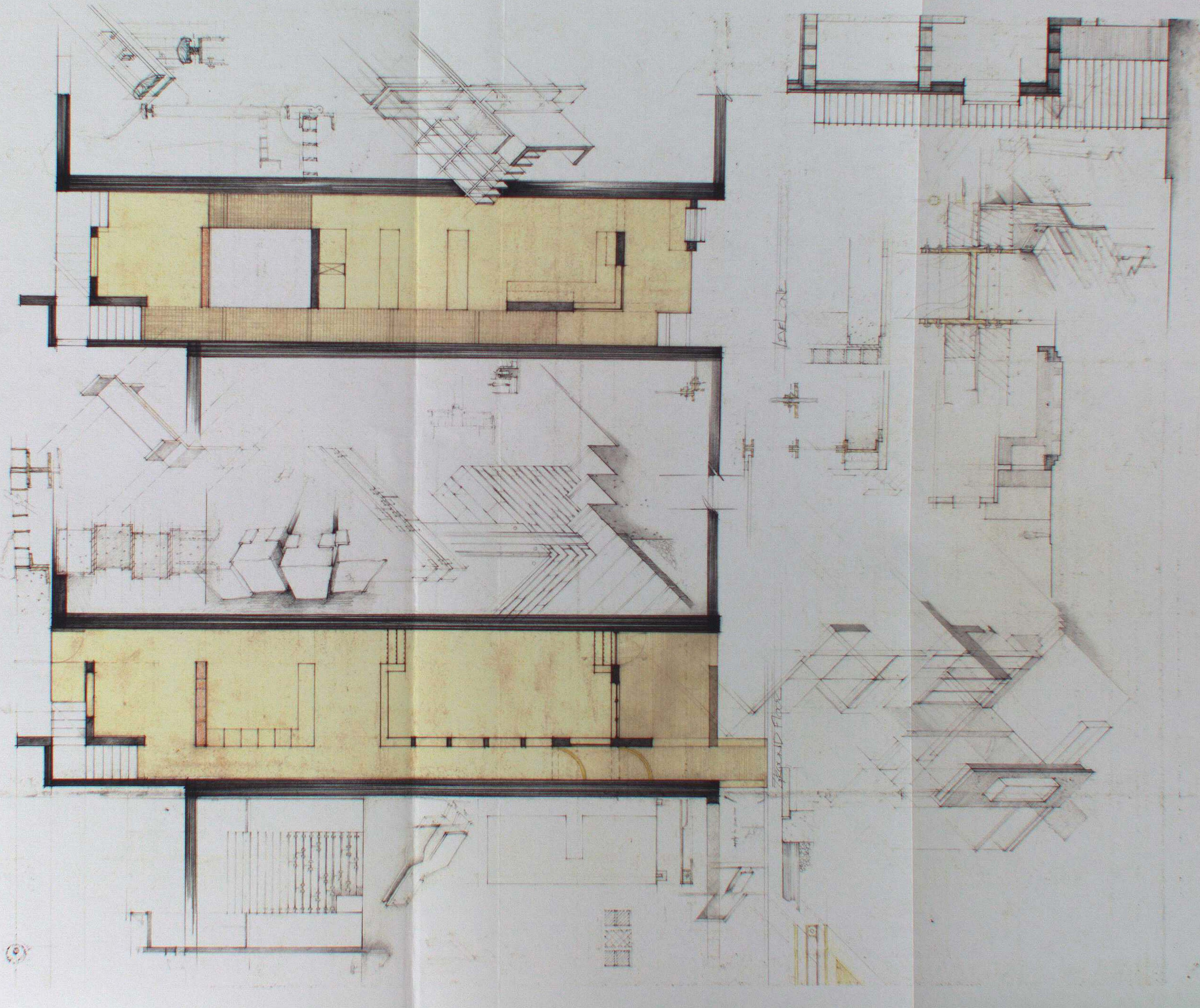



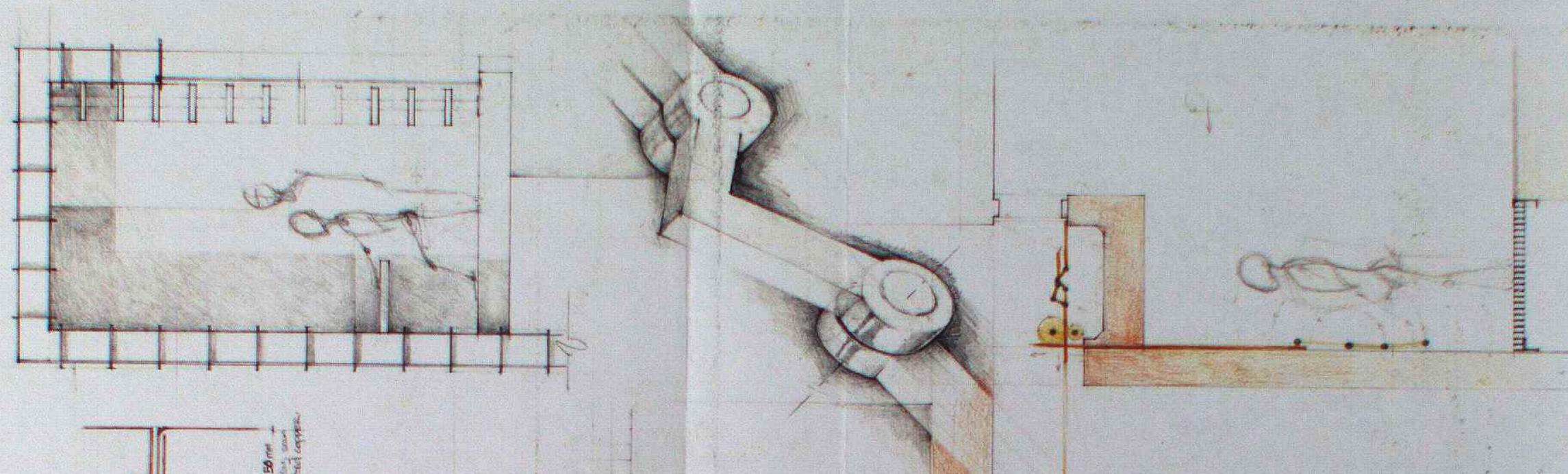

$$
\square \text { aा }
$$
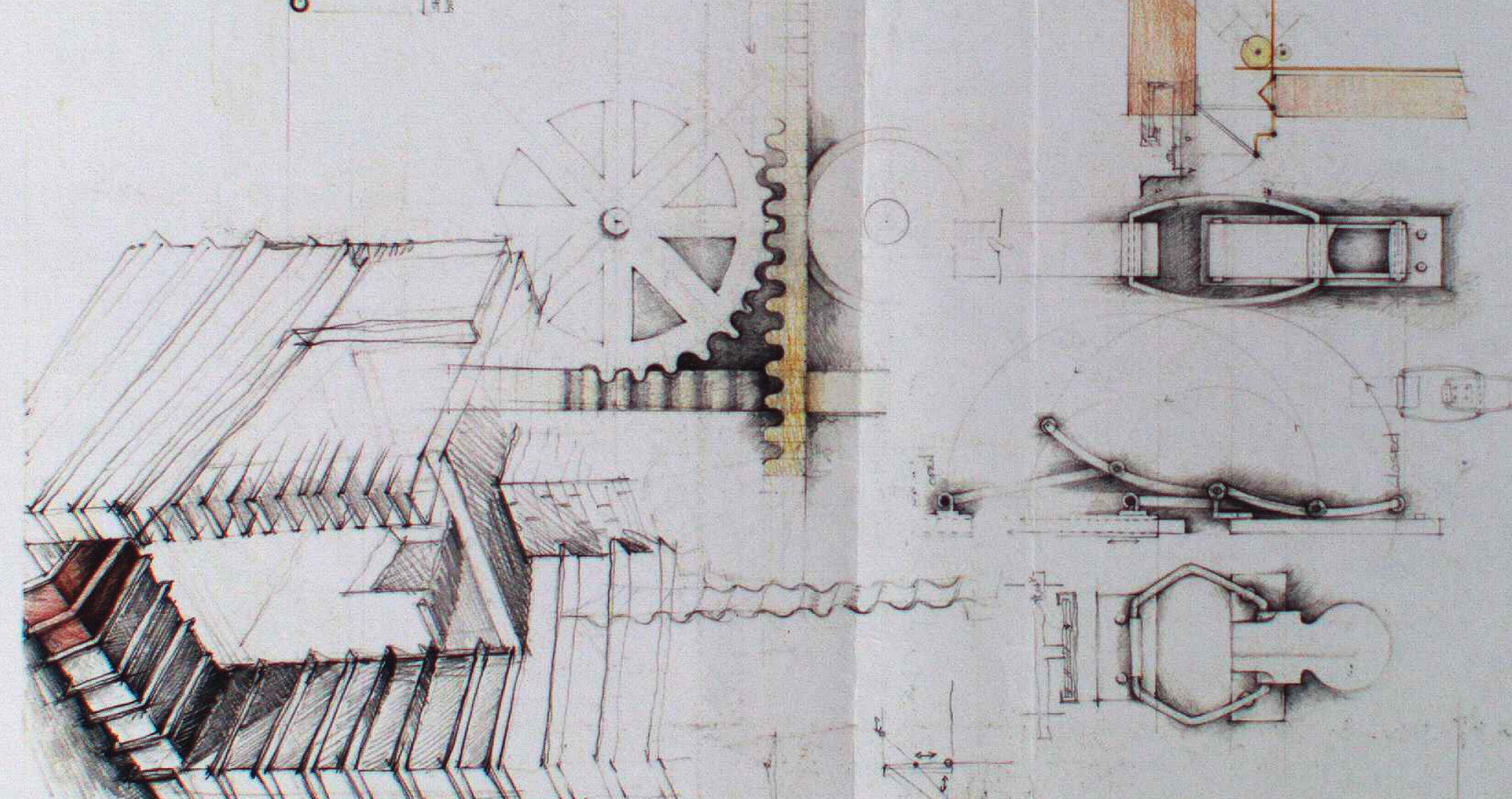

$1,1,1,1$
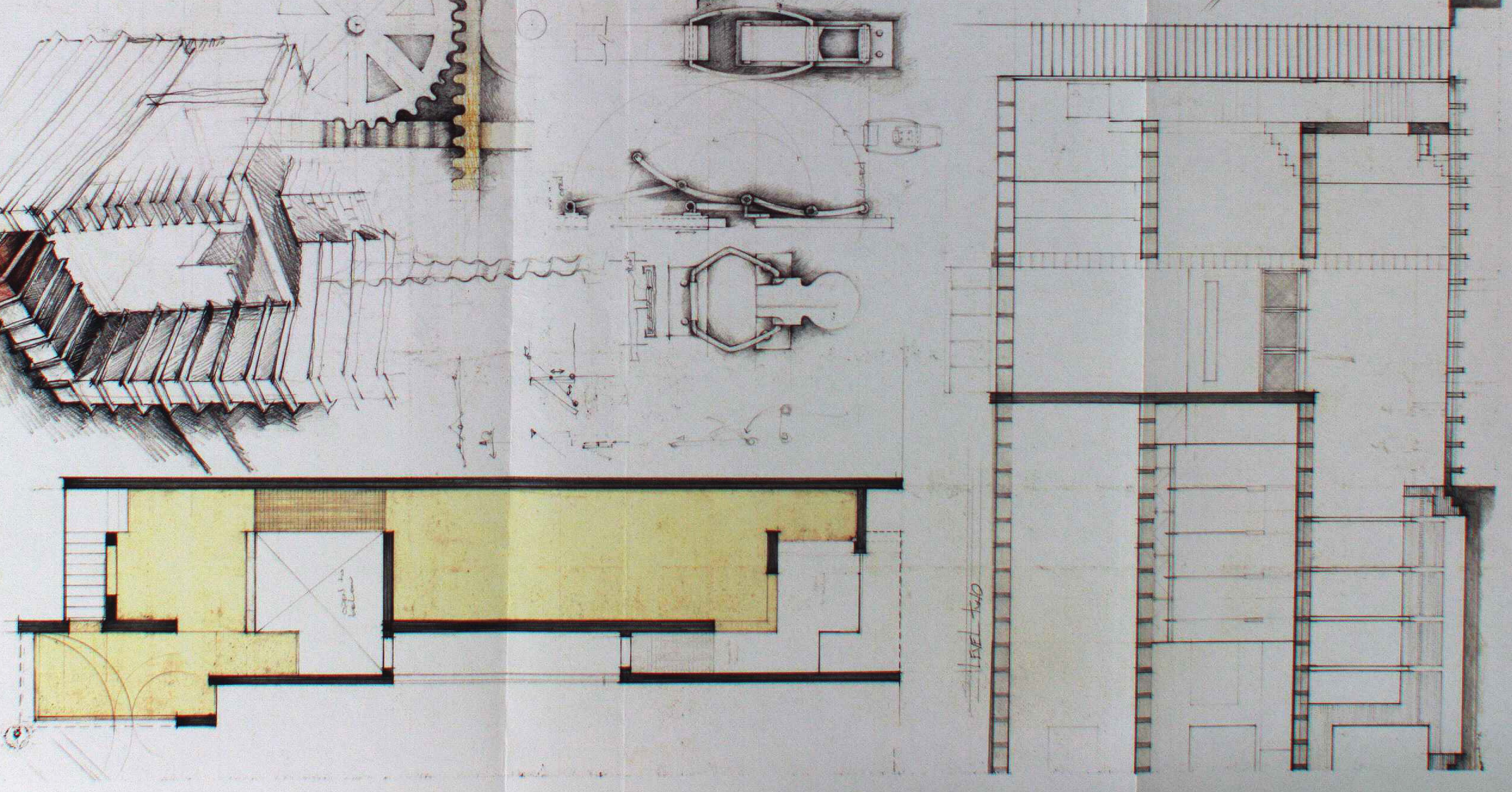


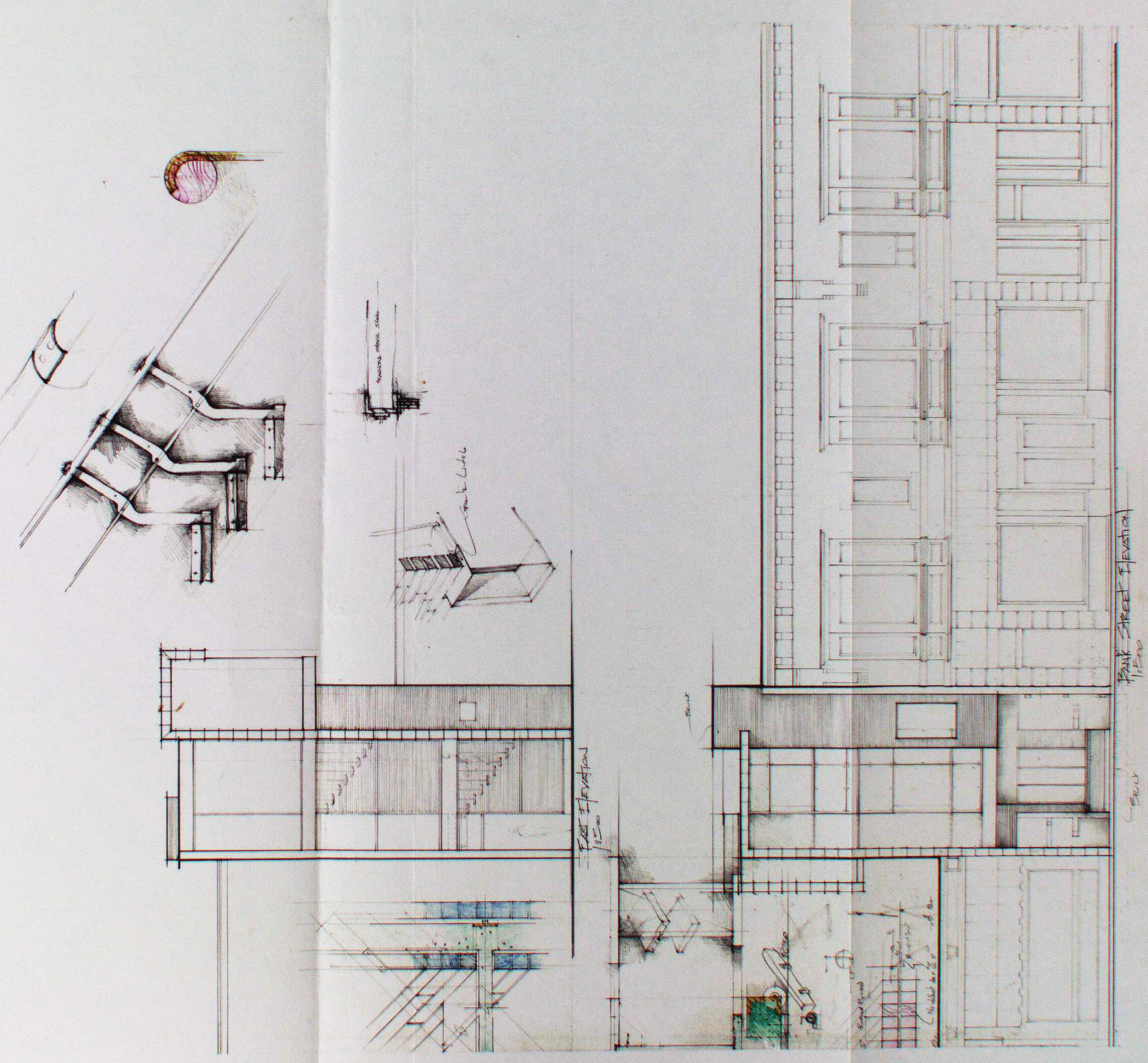

98 
